\title{
Wasserbewegung und Stofftransport in Pelo- solen am Beispiel des Südniedersächsischen Röt (Oberer Buntsandstein)
}

\author{
Dissertation \\ zur Erlangung des Doktorgrades \\ der Fakultät für Agrarwissenschaften \\ der Georg-August-Universität Göttingen
}

vorgelegt von

Clemens Stephan Siebner

geboren in Braunschweig

Göttingen, Februar 2000 
D 7

1. Referent: Prof. Dr. Brunk Meyer

2. Korreferent: Prof. Dr. Gerd Wessolek 


\section{Inhaltsverzeichnis}

Abbildungsverzeichnis...............................................................

Tabellenverzeichnis................................................................... IV

Verzeichnis der verwendeten Karten ................................................. VIII

Abkürzungsverzeichnis...........................................................

1 FRAGESTELLUNG 1

1.1 ALLGEMEINE FragestelLUNG 1

1.2 SPEZIELLE FRAGESTELLUNG 2

2 STAND DER FORSCHUNG 4

2.1 ZUR GENESE VON PELOSOLEN

2.2 WASSER- UND STOFFTRANSPORT IN SCHWEREN BÖDEN 5

2.2.1 TRANSPORTBEDINGUNGEN UND PARAMETER

2.2.2 MODELLENTWICKLUNG FÜR DEN WASSER- UND STOFFTRANSPORT IN

3 BESCHREIBUNG DES VERSUCHSSTANDORTES 13

3.1 GEOGRAPHISCHE LAGE 13

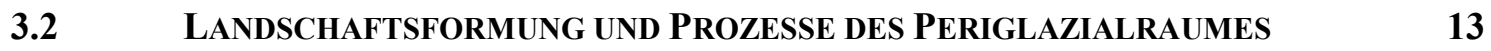

3.3 GEOLOGIE UND RÖT-STRATIGRAPHIE

3.4 DIE BÖDEN AUS RÖT

$\begin{array}{lll}\text { 3.4.1 BODENENTWICKLUNG AUF RÖT } & 18\end{array}$

3.4.2 BESCHREIBUNG DER BÖDEN AM VERSUCHSSTANDORT 20

4 FELDMESSUNGEN

4.1 UNTERSUCHUNGEN ZUM WASSERHAUSHALT UND ZUR WASSERBEWEGUNG 23

$\begin{array}{lll}4.1 .1 & \text { METHODIK } & 23\end{array}$

4.1.1.1 Arbeitsziele 23

4.1.1.2 Methodenwahl zur Messung der Wasserbewegung im Freiland 24

4.1.1.3 Modifizierung der Lysimetermethode für Freilandmessungen in Pelosolen27

4.1.2 ERGEBNISSE UND DISKUSSION 29

4.1.3 SCHLUSSFOLGERUNGEN ZUR ANWENDUNG VON PLATTENLYSIMETERN FÜR WASSERHAUSHALTSMESSUNGEN $\quad 36$

4.2 UNTERSUCHUNGEN ZUM STOFFTRANSPORT

4.2.1 METHODIK

4.2.1.1 Wahl des Tracers $\quad 37$

$\begin{array}{ll}\text { 4.2.1.2 Durchführung der Tracerversuche } & 38\end{array}$

4.2.2 ERGEBNISSE UND DISKUSSION ZUM SOLUTTRANSPORT 39

$\begin{array}{lll}\text { 4.2.2.1 Bromid-Tracer-Versuch } & 39\end{array}$

$\begin{array}{ll}\text { 4.2.2.2 Chlorid-Tracer-Versuch } & 40\end{array}$ 
4.2.3 BETRACHTUNGEN ZUM PARTIKELTRANSPORT 45

4.2.4 SCHLUSSFOLGERUNGEN UND AUSBLICK 48

5 BODENHYDROLOGISCHE UNTERSUCHUNGEN AN

MONOLITHISCHEN, WASSERGESÄTTIGTEN LABORLYSIMETERN 51

5.1 METHODIK

5.1.1 METHODENWAHL 51

5.1.2 WASSERLEITFÄHIGKEIT

5.1.3 CHLORID-DAUERTRACERVERSUCH 54

5.1.4 ZUR ABSCHÄTZUNG DER UNGESÄTTIGTEN WASSERLEITFÄHIGKEIT 56

5.2 ERGEBNISSE UND DISKUSSION

5.2.1 GESÄTTIGTE WASSERLEITFÄHIGKEIT

$\begin{array}{lll}5.2 .2 & & 60\end{array}$

5.2.3 ZUR ABSCHÄTZUNG DER UNGESÄTTIGTEN WASSERLEITFÄHIGKEIT 65

5.3 SCHLUSSFOLGERUNGEN AUS DEN LABORLYSIMETERVERSUCHEN

6 AUFNAHME DER FEINSTRATIGRAPHIE UND

BODENPHYSIKALISCHER PARAMETER

$\begin{array}{lll}\text { 6.1 } & \text { METHODIK } & \mathbf{7 5}\end{array}$

$\begin{array}{lll}6.1 .1 & \text { PROFILANSPRACHE } & 75\end{array}$

6.1.2 BODENPHYSIKALISCHE PARAMETER 76

6.1.2.1 Trockendichten 76

6.1.2.2 Wassergehalts-Saugspannungsbeziehung 77

6.1.2.3 Korngrößenanalyse 77

6.2 ERGEBNISSE DER FEINSTRATIGRAPHIE

6.2.1 AUFBAU DES GESAMTPROFILS 78

6.2.2 TIEFENVERTEILUNG VON PSEUDOSANDANTEILEN UND FREIEM CARBONAT 79

6.3 PROFILANSPRACHE $\mathbf{8 2}$

6.3.1 Allochthones Pelosol-AKKUMULATIONS-PROFIL 82

6.3.2 VERWITTERUNGSPROFIL 82

6.4 BODENPHYSIKALISCHE PARAMETER

6.4.1 DICHTEVERTEILUNGEN

6.4.2 WASSERGEHALTS-SAUGSPANNUNGSBEZIEHUNG 84

6.4.3 KORNGRÖßENVERTEILUNGEN $\quad 89$

$\begin{array}{ll}\text { 6.5 SCHLUSSFOLGERUNGEN } & 90\end{array}$

7 SCHLUSSBETRACHTUNG $\quad 92$

8 ZUSAMMENFASSUNG

9 LITERATUR 


\section{Abbildungsverzeichnis}

Abbildung 1: Beispiele für Durchbruchskurven von Tracern aufgetragen als relative Konzentration (C/Co) gegen das wassergefüllte Porenvolumen (PV) in Böden mit unterschiedlicher Porengrößenverteilung aus KUTílEK \& NIELSEN (1994): a: piston flow = Kolbenfluss, eine große kolbenförmige Pore, $b$ : normal distribution $=$ normale Porengrößenverteilung, $c$ : bi-modal distribution = 2-gipfelige Porengrößenverteilung S. 6

Abbildung 2: Ausschnitt aus der Topographischen Karte 1:25.000 (Blätter 4525 Reinhausen und 4526 Gellihausen)

Abbildung 3: Anordnung der Plattenlysimeteranlage am Kleinen Knüll; Breite der Ackerfläche $10 \mathrm{~m}$

Abbildung. 4: Tägliche Sickerwasserraten in den Plattenlysimetern in 30-90 cm Bodentiefe bei einer Beregnungsrate von $5 \mathrm{~mm}^{-1}$, Verdunstung ist ausgeschlossen

S. 34

Abbildung 5: Tiefenverteilung der Chloridkonzentrationen in der Bodenlösung neben dem $40 \mathrm{~cm}$ und dem $90 \mathrm{~cm}$ tiefen Plattenlysimeter; Lage: $75 \mathrm{~cm}$ neben dem Tracerstreifen

Abbildung 6: Chloridkonzentrationen in der Bodenlösung in Abhängigkeit von der Bodentiefe neben dem $60 \mathrm{~cm}$ tiefen Plattenlysimeter unter den Tracerstreifen (69 mm mit 1000 mg Chlorid je Liter

Abbildung 7: Chloridkonzentrationen in der Bodenlösung in Abhängigkeit von der Bodentiefe neben dem $70 \mathrm{~cm}$ tiefen Plattenlysimeter (Bohrung 47) unter einen Tracersteifen nach Beregnung von $70 \mathrm{~mm}$ mit $1000 \mathrm{mg}^{-1}$ Chlorid

Abbildung 8: Entwicklung der gesättigten Wasserleitfähigkeit bei innerer Erosion bei Lysimeter 30 mit 1,2 cm WS Druckdifferenz

Abbildung 9: Chlorid-Austragskurve: Chlorid-Konzentration $\left(\mathrm{mg}^{-\mathrm{L}^{-1}}\right)$ im Sickerwasser [mm] aus Pelosol-Lysimetern mit unterschiedlichen Längen ( Ly $1=30 \mathrm{~cm}$, Ly $2=53,1 \mathrm{~cm}$, Ly $3=67,5 \mathrm{~cm}$ ) bei dauerhafter Chlorid-Applikation (1000 $\left.\mathrm{mg}^{-1} \mathrm{~L}^{-1}\right)$ bei gesättigtem Fluss von $15 \mathrm{~mm}^{-1}$ 
Abbildung 10: Chlorid-Durchbruchskurven: $C / C o=$ relative Konzentration; $P V=$ Perkolat der Lysimeter ausgedrückt in Gesamtporenvolumen (Lysimeterlängen: $L y 1=30 \mathrm{~cm}$, Ly $2=53,1 \mathrm{~cm}$, Ly $3=67,5 \mathrm{~cm}$ )

Abbildung 11: Verlauf der Chlorid-Aufnahme des Porenraumes (\%-vol.) in Abhängigkeit vom Perkolationsvolumen ausgedrückt als Porenvolumen der Lysimeter (\%-vol.) (Lysimeterlängen: Ly $1=30 \mathrm{~cm}, \mathrm{Ly}$ $2=53,1 \mathrm{~cm}$, Ly $3=67,5 \mathrm{~cm}$ )

Abbildung 12: Ausfluss-Zeitfunktion aus den oberen $10 \mathrm{~cm}$ bei plötzlicher $A b$ senkung des Wasserspiegels bei dem $57,5 \mathrm{~cm}$ langen Bodenmonolith

Abbildung 13: Ausfluss-Zeitfunktion aus Lysimetern bei plötzlicher Absenkung des Wasserspiegels um $10 \mathrm{bzw} .8,5 \mathrm{~cm}$ S. 68

Abbildung 14: Lageskizze der Feldversuche am Kleinen Knüll bei Reinhausen 1997/98 (3 \% Hangneigung nach Süden)

S. 76

Abbildung 15: Röt-Pelosol Profilskizze am Kleinen Knüll mit den im Gelände erkennbaren Solifluktionslagen; eingezeichnet sind die Pseudosandanteile $(2000-63 \mu \mathrm{m})>20 \%$. Gew. und die Carbonatgehalte $>5 \%$-Gew.

$S 80$

Abbildung 16: Carbonatgehalte (CaCO3) und Summe der Pseudosandanteile (Sand \%-Gew.) in Abhängigkeit von der Bodentiefe in der Mitte des Profilgrabens

Abbildung 17: Tiefenverteilung der Trockendichte $\left(\mathrm{g} \mathrm{cm}^{-3}\right)$ im VerwitterterungsProfil (Cv) (Profil 1) und im tief verwitterten P-Horizont-Material (Profil 2)

Abbildung 18: Wassergehalts-Saugspannungs-Beziehung von wenig verwitterten Cv-Horizontmaterial (Pelit) aus 60 bis $125 \mathrm{~cm}$ Bodentiefe

S. 85

Abbildung 19: Wassergehalts-Saugspannungs-Beziehung vom gut verwitterten P-Horizontmaterial aus 35 bis $200 \mathrm{~cm}$ Bodentiefe S. 85

Abbildung 20: Tiefenverlauf der Porenvolumen (\%-Vol.) der Äquivalentporen $>30 \mu m$ im Pelosol-Akkumulationsprofil (P-Profil) und im Verwitterungsprofil (Cv-Profil) S. 88 


\section{Tabellenverzeichnis}

Tabelle 1: Vereinfachte Röt-Stratigraphie für das Südniedersächsische Bergland nach HINZE (1967) S. 16

Tabelle 2: $\quad$ Einteilungsmöglichkeiten und Entwicklungsreihen der Böden auf Tonsteinen (Pelosolen)

Tabelle 3: $\quad$ Zusammengefasste Merkmale einiger Pelosol-Bodenprofile am Versuchsstandort in Reinhausen am „Kleinen Knüll“; bei den Bodentiefen sind jeweils die Spannbreiten der Horizonte angegeben

Tabelle 4: $\quad$ Summen von Niederschlag $(N)$ und Sickerwasser $(S)$ aus tiefengestaffelten Plattenlysimetern in $30-90 \mathrm{~cm}$ Bodentiefe in $\mathrm{mm}$ nach der sommerlichen Austrocknung (Messbeginn: 1.10.96)

Tabelle 5: $\quad$ Monatssummen von Niederschlag $(N)$, Beregnung $(B)$ und Sickerwasser (S) aus tiefengestaffelten Plattenlysimetern in 30$90 \mathrm{~cm}$ Bodentiefe in $\mathrm{mm}$

Tabelle 6: $\quad$ Tageswerte von Niederschlag, Beregnung und Sickerwasser Anfang März 1997 (in mm)

Tabelle 7: $\quad$ Tiefenverteilung der Trockendichte, Wassergehalte, Gesamtporenvolumen und der Wasserziffer aus einer im Herbst umgegrabenen Fläche im Sommer 1997

Tabelle 8: $\quad$ Wiederfindungsraten von einem Bromid-Tracer-Puls mit 2000 mg $B r^{-} L^{-1}$ nach $187 \mathrm{~mm}$ Niederschlag und Beregnung.

S. 39

Tabelle 9: $\quad$ Tiefenverteilung der Wassergehalte und der daraus errechneten Bodenwassermenge in mm auf einer im Herbst 1996 umgegrabenen Fläche im Frühjahr 1997; Ackerkrume: 0-30 cm

Tabelle 10: $\quad$ Sickerwasserfrachten von braunem Ton $\left(g L^{-1}\right)$ in Plattenlysimetern verschiedener Bodentiefen und Niederschläge $(N)$ im Zeitraum vom 15.3.-2.4.1997 in g (leere Felder = nicht nachweisbar)

Tabelle 11: Druckdifferenzen bei der Messung der gesättigten hydraulischen Leitfähigkeit für die verschieden langen Laborlysimeter 
Tabelle 12: $\quad$ Berechnete Werte entwässerter Äquivalentporenbereiche (ÄPB) in Abhängigkeit von der Absenkungstiefe von der Oberfläche (AT) in einzelnen Bodentiefenabschnitten von vier Lysimetern (Lysim.)

Tabelle 13: Gesättigte hydraulische Leitfähigkeiten bei Pelosol-Lysimetern mit und ohne Solifluktionslage S. 60

Tabelle 14: Retentionsparameter der Bodenmonolithe: GPV = Gesamtporenvolumen (\%-Vol.); $P V(C l)=$ durch Chlorid ausgetauschtes Porenvolumen (\%-Vol.); $P V_{\text {ges }}=$ Anzahl durchflossener Porenvolumen; $1 . D B=$ erster messbarer Solut-Durchbruch

Tabelle 15: Gesamtporenvolumen (\%-Vol.) in Abhängigkeit von der Bodentiefe von 4 Bodenmonolithen unter Dauerbrache

Tabelle 16: $\quad$ Skelettanteil des Bodens (\%-Gew.) in Abhängigkeit von der Bodentiefe von 4 Bodenmonolithen unter Dauerbrache S. 70

Tabelle 17: Mittelwerte und Variationskoeffizient (Vk.) vom Skelettanteil und Gesamtporenvolumen (GPV) von vier Bodenmonolithen in verschiedenen Tiefenabschnitten

Tabelle 18: Berechnete Werte entwässerter Äquivalentporenbereiche (ÄPB) und Messwerte des entwässerten Porenvolumens (PV) in Abhängigkeit von der Absenkungstiefe (AT) in einzelnen Bodentiefenabschnitten von vier Lysimetern (Zahl in Klammern ist die Absenkungstiefe des Wasserspiegels von der Bodenoberfläche)

S. 72

Tabelle 19: Profilaufbau in der Mitte vom Profilgraben (Allochthones PelosolAkkumulations-Profil)

Tabelle 20: Profilaufbau in der Mitte vom Profilgraben (Verwitterungs-Profil)

Tabelle 21: Kapillarwasserverteilung vom P-Akkumulationsprofil (P-Profil) und vom Verwitterungsprofil (Cv-Profil) aus Pelit: $\ddot{A} D=A ̈$ quivalentdurchmesser in $\mu \mathrm{m} ; \%$-Vol. = volumetrischer Wassergehalt; $n F K=$ nutzbare Feldkapazität in \%-Vol.; VP = Verfügbarkeit für pflanzliches Wasser

S. 86 
Tabelle 22: Korngrößenverteilung (\%-Gew.) vom Pelosol-Akkumulations- $(P$ Profil) und Verwitterungsprofil (Cv-Profil). o. Z. = ohne Zerstörung der Kittsubstanzen; $m$. Z. = mit Zerstörung der Kittsubstanzen

S. 89

Tabelle 23: Korngrößenverteilung (\%-Gew.) vom Pelosol-Akkumulations- (PProfil) und Verwitterungsprofil (CV-Profil) ohne Zerstörung der Kittsubstanzen ohne carbonathaltige Proben; $Q_{T}=$ Quotient aus Totwasservolumen (\%-Vol.) und Tongehalt(\%-Gew.) $\quad$ S. 90 


\section{Verzeichnis der verwendeten Karten}

\section{Topographische Karten:}

NIEDERSÄCHSISCHES LANDESVERWALTUNGSAMT-

LANDESVERMESSUNG (Hrsg.):

Topographische Karte 1:25.000, Blatt 4525 Friedland,

Topographische Karte 1:25.000, Blatt 4526 Gleichen

\section{Geologische Karten:}

NIEDERSÄCHSISCHES LANDESVERWALTUNGSAMT-

LANDESVERMESSUNG (Hrsg.):

Geologische Karte 1:25.000, Blatt 4525 Reinhausen, 1893

Geologische Karte 1:25.000, Blatt 4526 Gellihausen, 1883

Geologisches Blockbild der Umgebung von Göttingen, Veröff. d. Niedersächs.

Institut f. Landeskde. u. Landesentwicklung a. d. Univ. Göttingen von U. Nagel \& H.-G. Wunderlich, 1976

Bodenkundliche Karten:

NIEDERSÄCHSISCHES LANDESAMT FÜR BODENFORSCHUNG

Digitale Bodenkarte $1: 50.000$ und Bodenübersichten, Hannover, 1997 


\section{Abkürzungsverzeichnis}

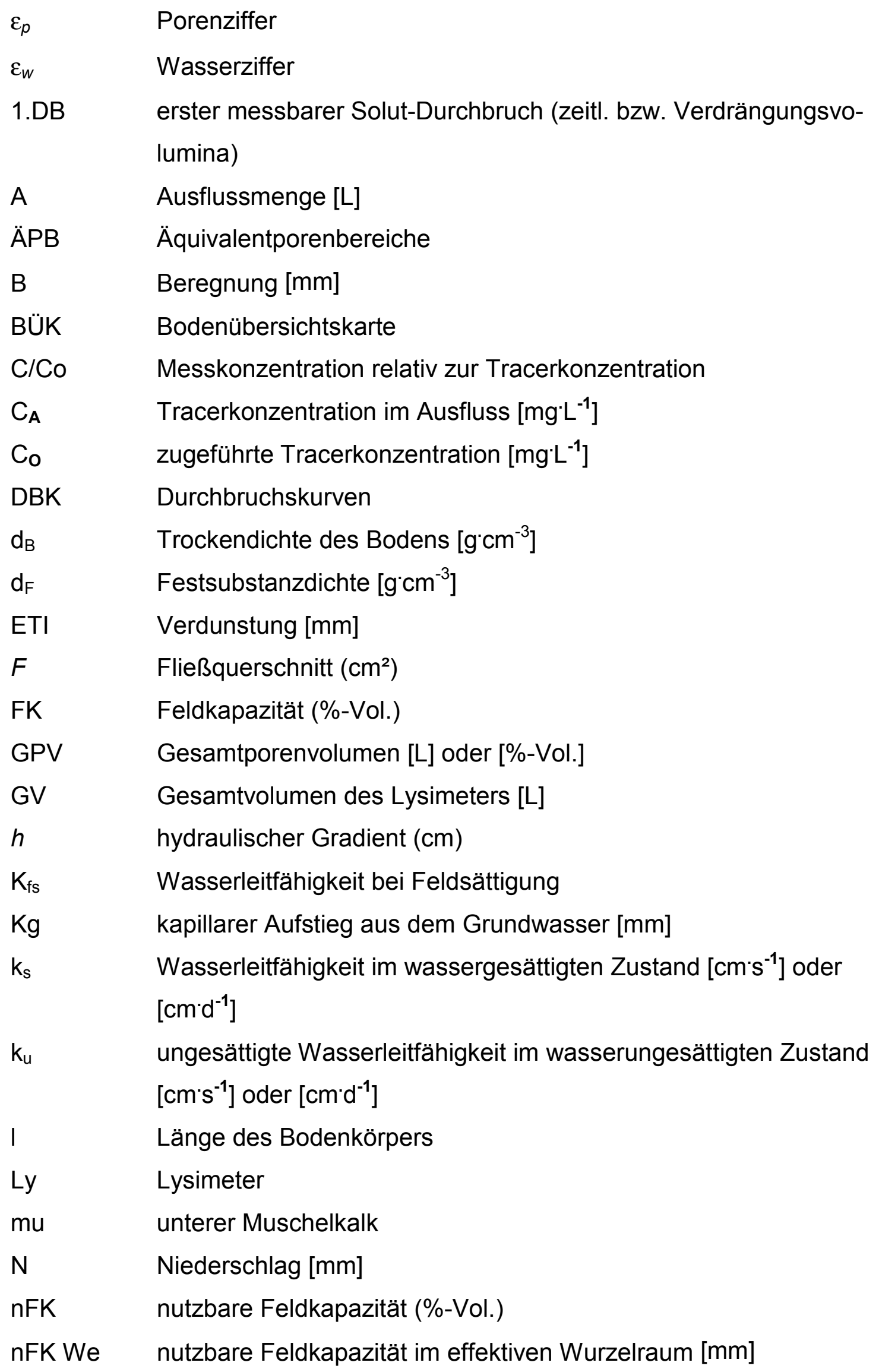


O Zugeführte Wassermenge [L]

$\mathrm{PV}\left(\mathrm{Cl}^{-}\right) \quad$ durch Chlorid ausgetauschtes Porenvolumen (\%-Vol.)

PV Porenvolumen $\left[\mathrm{cm}^{3}\right]$

$\mathrm{PV}_{\text {ges }} \quad$ Anzahl theoretisch ausgetauschter Porenvolumen

PWP Permanenter Welkepunkt [\%-Vol.]

$\mathrm{Qb} \quad$ Zwischenabfluss (Interflow) [mm]

Qo Überlandfließen [mm]

S Sickerwasserbildung (kurzfristige Grundwasserbildung) [mm]

sm mittlerer Buntsandstein

so oberer Buntsandstein

$\mathrm{t} \quad$ Zeit [d]

WS Wassersäule [cm]

V Wasservolumen $\left(\mathrm{cm}^{3}\right)$

Var. Variationskoeffizient

$V_{\text {fest }} \quad$ Volumen der Festsubstanz [L]

$\mathrm{V}_{\mathrm{w}} \quad$ wassergefülltes Porenvolumen [L]

$\Delta \mathrm{F} \quad$ Wassertransport in der bodennahen Luft [mm]

$\Delta \mathrm{Vb} \quad$ Änderung des Wasservorrats im Boden [mm]

$\Delta$ Vo $\quad$ Änderung des Wasservorrats an der Erdoberfläche [mm] 


\section{Fragestellung}

\subsection{Allgemeine Fragestellung}

Die Messung von Wasser- und Stoffflüssen in Böden dient der Erfassung des Haushaltes. Die zentrale Frage ist, ob ein Stoff im Boden ab- oder angereichert wird und in welcher Richtung er verlagert wird.

Zu Beginn der landwirtschaftlichen Bodenforschung galt das Interesse ausschließlich den für das Pflanzenwachstum relevanten Größen. Der Boden als Vermittler von Wasser- und Nährstoffen stand im Vordergrund, um möglichst hohe Erträge zu produzieren. Das über die Verdunstung hinaus fallende Niederschlagswasser wird aus dem Boden ausgewaschen und führt zur Verlagerung von Stoffen, die letztlich in das Grundwasser oder Oberflächengewässer gelangen können.

Die bodenphysikalische Forschung der letzten Dekaden hat sich mit Flussmessungen und Stoffhaushalten von Böden beschäftigt. Ziel solcher Untersuchungen ist es, Kenntnisse über die Transportmechanismen des Stofftransportes zu gewinnen. Man versucht dann Simulationsmodelle zu entwickeln, um die an einem Objekt gewonnenen Erkenntnisse zu verallgemeinern. Diese Simulationsmodelle sind oft nicht hinreichend genau, da der Boden als dreiphasiges System in seiner räumlichen Anordnung schwer zu beschreiben ist.

In dichtbesiedelten Räumen, wie in Mitteleuropa, greift der Mensch tief in die natürlich ablaufenden Prozesse ein. Der Grundwasservorrat wird durch die hohe Bevölkerungsdichte stark beansprucht. Die grundlegende Veränderung der Landnutzung greift stark in den Wasserhaushalt ein. Der Boden spielt als Vermittler zwischen der Atmosphäre und den Grundwasservorräten eine zentrale Rolle. Einerseits beeinflusst der Boden die jährlich neu gebildete Sickerwassermenge und damit die Grundwasserneubildung, andererseits das Transportverhalten für eingetragene Stoffe. Die Transportparameter des Wasser- und Stofftransportes sind in den letzten drei Dekaden intensiv untersucht worden. Für Pelosole sind sie jedoch nicht hinreichend genau ermittelt (KENKEL, 1997). Hier soll die vorliegende Arbeit ansetzen. 


\subsection{Spezielle Fragestellung}

Die Funktion von Peliten und aus innen entwickelten Böden für den Wasserund Nährstoffhaushalt im Mitteldeutschen Oberen Buntsandstein (Röt) ist unklar. Bei Untersuchungen zum Landschaftswasserhaushalt wird davon ausgegangen, dass Tonsubstrate eine hydrologische Barriere bilden, weil die gesättigte Wasserleitfähigkeit der Matrix durch die Textur sehr gering ist. Dennoch sind keine Anzeichen für oberflächlichen Abfluss bzw. rezente Erosion zu erkennen. Außerdem gibt es nach eigenen Beobachtungen im Göttinger Raum im Röt keine Quellen. Da im Gelände keine Anzeichen für nennenswerten Oberflächenabfluss vorhanden sind, stellt sich die Frage nach der Infiltration und der Tiefensickerung. Der Arbeitsansatz der Gebietswasserbilanz für Rötgebiete geht davon aus, dass keine Tiefensickerung möglich ist, so dass das betreffende Gebiet im hydrologischen Sinne geschlossen ist. Hier ist also die Frage zu beantworten, ob es im Sinne des Landschaftswasserhaushaltes hydrologisch geschlossene Rötgebiete gibt, und wo das Sickerwassers verbleibt.

Die Gebietswasserbilanz eines Rötgebietes ergab einen Bilanzüberschuss (WILHELMI, 1994), der auf unterirdischen Zufluss zurückgeführt wurde. Eine andere Arbeit konnte in mehreren Jahren keinen einheitlichen Wasservorratswert auf Landschaftsebene ermitteln (PÖRTGE, 1979). Die Jahressummen für Gebietswasserabfluss und Niederschlag zeigten keinen Zusammenhang. Die Anwendung verschiedener Modelle für den Landschaftswasserhaushalt in einem Rötgebiet (KENKEL, 1997) zeigte, dass die hydrologischen Eingabeparameter unklar sind. PÖRTGE (1996) fand tagesperiodische Schwankungen der hydraulischen Parameter. Die Frage nach der Funktion von Pelosolen im Landschaftswasserhaushalt und die Eigenschaften der Wasserhaushaltskomponenten steht deshalb im Mittelpunkt dieser Arbeit. Ziel ist es, für ein Normaljahr die Wasserhaushaltsbilanz aufzustellen.

Im Göttinger Raum gibt es einige Wassereinzugsgebiete, in denen Röt anzutreffen ist. Unter dem Röt befindet sich der mittlere Buntsandstein (sm), ein klüftiger Sandstein. Dieser bildet einen ergiebigen Aquifer, der häufig von der Trinkwassererzeugung genutzt wird. Wenn die Rötgebiete nicht als geschlossen angesehen werden können, bleibt die Frage, ob Wasser aus dem Röt in den mittleren Buntsandstein sickern kann. Damit hätte der Röt für die Wasserwirtschaft eine viel größere Rolle, als ihm bislang zugesprochen wird. 
Eng gekoppelt an den Wassertransport ist der Stofftransport. Zum einen werden Stoffe direkt vom Wasser gelöst und mitgeführt. Diese Form des Transportes wird als Solut- oder Lösungstransport bezeichnet. Zum anderen können suspendierte Partikel verlagert werden. Zu nennen sind Pflanzenschutzmittel und Bakterien, z.B. aus der Gülleausbringung. Zunächst stellt sich die Frage, ob Stofftransport in Pelosolen und aus den Pelosolen hinaus in das Grundwasser möglich ist. Ist dies der Fall, dann sollen die Transportparameter für eine möglichst große Skala ermittelt werden.

Böden aus Tonsubstraten schrumpfen beim Austrocknen und quellen bei der Wasseraufnahme. Im trockenen Zustand gibt es tiefe Schrumpfrisse, in denen ein bevorzugter Wasser- und Stofftransport möglich ist. MACKIE et al. (1984) berichten, dass beim Quellen eines schweren Tonbodens die Makroporen überwiegend schließen. Demzufolge muss der Quellungszustand einen großen Einfluss auf den Wasser- und Stofftransport haben. Die Auswirkungen des Quellungszustandes auf die Tiefeninfiltration sollen untersucht werden.

Für Belange der landwirtschaftlichen Praxis in Wassereinzugsgebieten soll die Keim- und die Nitratverlagerung bzw. Auswaschung bestimmt werden. 


\section{Stand der Forschung}

Die Tonfraktion von Böden umfasst definitionsgemäß die Korngrößen $<2 \mu \mathrm{m}$. Die Texturporen von Tonsubstraten haben deshalb einen kleinen Durchmesser und zeichnen sich durch äußerst geringe gesättigte Wasserleitfähigkeiten aus (DE MOL, 1996). Aus diesem Grund werden Tone bei der Abdichtung oder als Unterlage von Deponien genutzt. Undichtigkeiten an Deponien haben in der Vergangenheit gezeigt, dass die Dichtigkeit von Tonen oft überschätzt wird und die Eignung für Deponieabdichtungen in Frage gestellt werden muss (KRAKOW, 1990).

Der Arbeitsansatz der Gebietswasserbilanz auf Landschaftsebene setzt voraus, dass unter geschlossenen Pelit-Gebieten die Gebietswasserbilanz aus oberflächlichen Zu- und Abflüssen ermittelt werden kann. Es wird von der geringen Wasserleitfähigkeit der wassergesättigten Tone als hydrologische Barrieren ausgegangen (HÖLTING, 1992).

\subsection{Zur Genese von Pelosolen}

Pelosole entstehen bei der Bodenbildung aus Pelitgestein. In Peliten sind die Tonteilchen diagenetisch verfestigt (MÜCKENHAUSEN, 1985). Dadurch ist die hydrologisch wirksame Körnung im Schluff- bis Feinsandbereich, also größer als die der einzelnen Tonteilchen. Pelite können deshalb wasserdurchlässig sein. Erst im Zuge der Verwitterung entsteht plastische Tonsubstanz mit geringer Korngröße, die dann für Wasser nahezu undurchlässig ist und bei der Deponieabdichtung benutzt wird.

Die Verwitterung der Pelite erfolgte im Göttinger Raum vor allem im periglazialen Klima der letzten Eiszeiten. Hier ist die Verwitterung an vielen Stellen durch Solifluktion unterbrochen, dadurch entstehen Wechsellagen aus verwittertem Rötmaterial im Wechsel mit weniger verwittertem Material. Folglich ist an vielen Stellen kein durchgehender P-Horizont mit Tongehalten $>45 \%$, wie in der pedogenetisch gebräuchlichen Definition (AG BODEN, 1994; AK BODENSYSTEMATIK, 1998), vorhanden. Geringe P-Horizontmächtigkeit kommt auch durch Abtrag, Solifluktion oder bei der Vermischung des P-Horizontes mit weniger verwittertem Material zustande. Die feldgesättigte Wasserleitfähigkeit $\left(\mathrm{k}_{\mathrm{fs}}\right)$ ist in einem solchen Falle wesentlich höher als man nach der Textur erwarten würde 
(BRANDT, 1993). Für tonige Böden gibt die Bodenkundliche Kartieranleitung (AG BODEN, 1994) mittlere wassergesättigte Leitfähigkeiten von $2-20 \mathrm{~cm}^{\prime} \mathrm{d}^{-1}$ an, und stuft die Leitfähigkeit als mittel bis gering ein. Pelitlandschaften mit geringer verwitterter Pelitauflage können jedoch einen Beitrag zur Grundwasserbildung leisten (BRANDT et al., 1992).

Der Boden ist ein Gemisch aus gasförmiger, flüssiger und fester Phase. Zwischen flüssiger und fester Phase tritt Adhäsion auf, durch die es bei abnehmenden Wassergehalt zur Schrumpfung, also zum Volumenverlust des Bodenkörpers kommt. Da Tone beim Trocknen schrumpfen, entstehen durch den Volumenverlust Risse, in denen Wasser schnell eindringen kann. Tone unter einer Deponie müssen deshalb feucht bleiben, um die Dichtigkeit zu erhalten (NASSAR et al., 1996; KRAKOW 1990; DÖLL, 1996). Trockenrisse entstehen auch, wenn der Ton vorher künstlich verdichtet worden ist (SHACKELFORD et al., 1989).

Durch die Schrumpfung bildet sich ein Netz von Schrumpfrissen, welches in trockenen Jahren beträchtliche Tiefen erreichen kann (Mc KENZIE et al., 1994). Die Infiltration von Wasser und darin enthaltenen Stoffen geht bei ausgetrockneten Tonböden entlang der Schrumpfrisse bis in größere Tiefen, wie mit Farbstoffinfiltrationsversuchen nachgewiesen werden konnte (BOUMA \& DEKKER, 1978; BoUMA et al., 1978). HEYDER (1993) führte die hohe räumliche Variation der Nitratgehalte in einem Tonboden auf Makroporenfluss zurück.

\subsection{Wasser- und Stofftransport in schweren Böden}

\subsubsection{Transportbedingungen und Parameter}

Für den Transport gelöster und suspendierter Stoffe in Makroporen gibt WHITE (1985) eine ausführliche Übersicht. Der Transport von Bakterien und Viren in Böden ist von BITTON \& HARVEY (1992) zusammengefasst worden.

Infiltrationsversuche mit Infiltrometern dienen der Messung der gesättigten Leitfähigkeit $\left(\mathrm{k}_{\mathrm{s}}\right)$, die die maximale Infiltrationsrate in den Boden darstellt. Aufgrund des unterschiedlichen Quellungszustandes des Bodens und der damit verbundenen Änderung des Porenvolumens variiert die feldgesättigte Wasserleitfähigkeit im Jahresgang (BRANDT, 1993). MESSING \& JARVIS (1990) haben durch Messung der $\mathrm{k}_{\mathrm{fs}}$ ein Schrumpfmodell der Inter-Aggregat-Makroporosität entwickelt, welches die $\mathrm{k}_{\mathrm{fs}}$ in Abhängigkeit vom gravimetrischen Wassergehalt 
vorhersagt. Entscheidend ist dabei, dass die sommertrockenen Böden zwar für die Messung vorbefeuchtet werden, jedoch die Messung schneller als der Quellungsvorgang abgeschlossen ist.

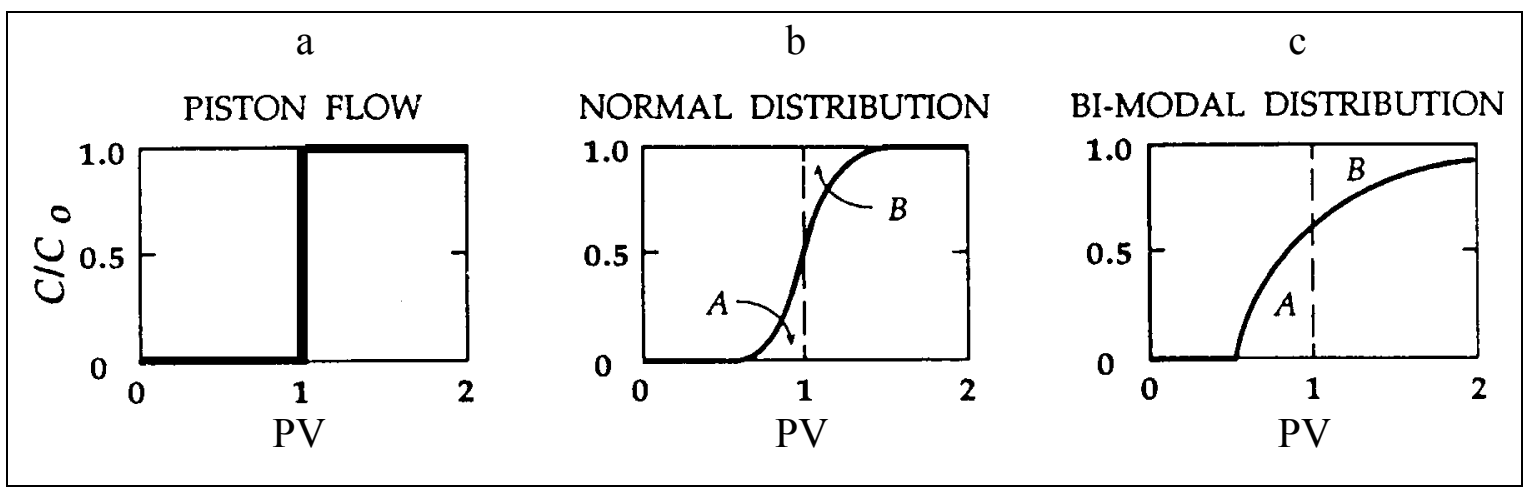

Abbildung 1: Beispiele für Durchbruchskurven von Tracern aufgetragen als relative Konzentration (C/Co) gegen das wassergefüllte Porenvolumen (PV) in Böden mit unterschiedlicher Porengrößenverteilung aus KUTÍLEK \& NIELSEN (1994): a: piston flow $=$ Kolbenfluss, eine große kolbenförmige Pore, $b$ : normal distribution $=$ normale Porengrößenverteilung, $c$ : bi-modal distribution = 2-gipfelige Porengrößenverteilung

Wasserbewegung und Stoffverlagerung hängen von verschiedenen Faktoren ab. Wichtig ist die Gestalt des wassergefüllten Porenraumes, in der sich, je nach Bodenfeuchte, unterschiedliche Anteile von Wasser und Luft befinden. Die Gestalt des Porenraumes und ihre Auswirkungen auf den Stofftransport werden in unterschiedlichen Modellvorstellungen beschrieben. Für die Verlagerung von gelösten Stoffen wird dann davon ausgegangen, dass z.B. Wasser ohne gelöste Salze durch eine Salzlösung aus dem wassergefüllten Porenvolumen verdrängt wird (Abbildung 1). Die einfachste Modellvorstellung für den Porenraum ist der Kolbenfluss (,,piston flow“) (Abbildung 1a). Die Pore, durch die das Wasser fließt, ist ein Zylinder. Der Konzentrationsanstieg des Ausflusses verläuft durch den Vorschub des Kolbens sprunghaft nach der Wassermenge von einem Porenvolumen auf die Konzentration der Salzlösung. Das gesamte Porensystem im Boden besteht jedoch aus vielen verschiedenen Porengrößenklassen, wobei in jeder Porengrößenklasse bei gleichen Potentialgradienten eine 
andere Geschwindigkeit herrscht. Es kommt dazu, dass der Konzentrationsanstieg in den Durchbruchskurven 1b und 1c insgesamt langsamer und schon vor einem durchflossenem Porenvolumen stattfindet. Wegen der unterschiedlich langen Weglängen durch die einzelnen Poren entsteht deshalb vermischte Verdrängung („miscible displacement“) oder Dispersion. In Abbildung 1b ist die Durchbruchskurve durch einen porösen Körper mit einer monomodalen Porengrößenverteilung aufgetragen. Monomodal bedeutet, dass die Porengrößenverteilung nur ein Maximum hat, wenn man die Porengröße gegen das anteilige Volumen aufträgt. In strukturierten Böden wird von einer bimodalen Porengrößenverteilung (Abbildung 1c) gesprochen (GERKE \& VAN GENUCHTEN, 1993), d.h. dass es zwei Maxima in der Porengrößenverteilung gibt: Texturbedingt bei den Poren aus der Matrix und strukturbedingt aus Einflüssen der Bodenbildung, wie Schrumpfrissen oder aus Regenwurmgängen. Die Matrixporen haben einen geringen Durchmesser, die Strukturporen zählen zu den Makroporen. Zu erwarten ist, dass das Wasser in den Makroporen sehr schnell fließt und der erste Konzentrationsanstieg in der Durchbruchskurve schon relativ schnell kommt. Die Endkonzentration wird erst sehr spät erreicht, da die Fließgeschwindigkeit in der Matrix gering ist.

Makroporen haben auch auf den Stofftransport besondere Auswirkungen. Das miscible displacement ist für homogene Böden und Grundwasserleiter lange bekannt (Nielsen \& Biggar, 1961; Biggar \& Nielsen, 1961; VAN DER Ploeg \& BENECKE, 1972; SCHRÖDTER, 1983). Die Bedingungen für „miscible displacement“ gelten nach BOUMA \& DEKKER (1978) nicht für strukturierte Tonböden.

Bei der Modellierung des Stofftransportes in Böden wird angenommen, dass es sich um ein homogenes, isotropes Medium handelt. Dieser Ansatz hat sich in der Vergangenheit nur teilweise bewährt (BOHNE, 1996). Bereits in Böden mit einer relativ gleichmäßigen Porengrößenverteilung reicht die rein dispersive Beschreibung des Transportes nicht mehr aus (BEESE \& VAN DER PLOEG, 1979).

Bei Modellen für den Wasser- und Stofftransport in natürlich gelagerten Böden gibt es das Konzept vom 2-phasigen System mit mobilem und immobilem Wasser (DE SMEDT et al., 1981). Dabei werden die verschieden starken Bindungen des Bodenwassers an die Festsubstanz berücksichtigt. Die Folge ist, dass sich das Bodenwasser bei einem bestimmten Potentialgradienten im Boden in un- 
terschiedlichen Geschwindigkeiten bewegt, wobei ein Teil des Bodenwassers immobil ist. Aus der mobilen Phase gelangt ein Teil der gelösten Stoffe über Diffusion in die immobile Phase und bei entsprechendem Konzentrationsgradienten diffundiert es wieder zurück. Mobile und immobile Phasen sind in strukturierten Böden der Inter- und Intra-Aggregatraum (SCHLINDWEIN, 1992). In Sandböden wurde die immobile Phase in den Humusaggregaten und die mobile Phase in der mineralischen Substanz gefunden (STIEGEMANN, 1991).

Grundannahme für das Modell des dispersiven Stofftransport ist die, dass im Porensystem laminare Flussbedingungen herrschen (HARTGE \& HORN, 1991), wobei die Flussgeschwindigkeit an der Porenwand gegen Null geht. Das Fließfeld breitet sich in der Pore in einer parabolischen Form aus. LOGSDON (1995) zeigte, dass in einer Makropore mit $6 \mathrm{~mm}$ Durchmesser der Fluss nicht laminar sein kann, sondern nach den Gesetzen der Turbulenz folgt. Es entsteht eine lineare Ausbreitungsfront senkrecht zur Fließrichtung. Das Dispersionsmodell gilt daher nicht für die Stoffverlagerung in Böden mit Makroporen. Makroporen spielen beim Stofftransport bei nahezu wassergesättigten Bedingungen in Lössböden eine herausragende Rolle. In Beregnungsversuchen bei Wassergehalten nahe der Feldkapazität fand BEISECKER (1993) 80\% der Infiltration in Makroporen. LOGSDON (1995) fand schon bei geringen Fließgeschwindigkeiten in einem tonigen Lehm bevorzugten Abfluss in einer Makropore. Bei diesen Versuchen zeigte sich der bevorzugte Abfluss auch, bei künstlicher Homogenisierung der oberen $12 \mathrm{~cm}$ Boden vor dem Versuch. Ähnlich sind die Ergebnisse von GÄTH et al. (1996) für Bromid-Verlagerung in Löss mit künstlichen Makroporen und einer mechanischen Porenwandverdichtung zur Simulation von Regenwurmgängen.

BOUMA \& DEKKER (1978) konnten zwar präferentielle Flüsse in Makroporen mit Methylen-Blau sichtbar machen, eine Bilanz war jedoch aufgrund der Sorption des Farbstoffes an die Bodenmatrix nicht möglich. Die Auswirkungen von Makroporen auf den Stofftransport untersuchten BECHER (1985) sowie GROENVELT \& BOLT (1972), mittels tiefengestaffelter Tensionsmessungen. Alle Autoren konnten die bevorzugte Infiltration in wenigen Bahnen anhand eines sägezahnähnlichen Verlaufes der Tensionskurven in Tiefen $>80 \mathrm{~cm}$ nach größeren Niederschlägen feststellen. Die verlagerten Wassermengen bleiben dabei unbekannt. 
BECHER (1985) gibt eine Vorstellung über zeitliche und räumliche Variabilität der mobilen und immobilen Phase: Im teilgesättigten Tonboden ist die Makropore luftgefüllt und deshalb Bestandteil der immobilen Phase, die das Filmwasser und die engen Matrixporen umfasst. Die mobile Phase ist ausschließlich in den etwas weiteren Matrixporen zu finden. Der Fluss folgt dem Gradienten vom höherem zum niedrigerem Feuchtigkeitsgehalt. Dieser Fluss ist deshalb mit Modellen, die auf dem Darcy-Gesetz aufbauen, zu beschreiben. Anders der wassergesättigte Zustand: Die mobile Phase befindet sich in den wassergefüllten Makroporen, während in den Matrixporen durch Abnahme des Wasserspannungsgradienten keine Wasserbewegung mehr zu finden ist. Im gesättigten Zustand ist die Filtration von Stoffen wegen der hohen Fließgeschwindigkeit in der Makropore sehr gering. Der eigentliche Filterkörper des Bodens, die Matrix, wird dadurch umgangen.

Die in der Vergangenheit auf Pelosolen durchgeführten Tracerversuche hatten geringe Wiederfindungsraten (SCHWEIKLE, 1982). Die erreichten Endkonzentrationen waren verglichen mit anderen Tracerstudien (GAESE, 1978; ROGOWSKI, 1988) sehr gering. Als Ursache ist die Diffusion in die immobile Phase hinein, oder die langsame Wasserbewegung in der Matrix anzunehmen.

Chloridtracer- und Farbstoffversuche auf ausgetrockneten Tonböden zeigten, dass bei Applikation des Farbstoffes einige Zeit vor der Wasserinfiltration die Matrix den Stoff fast vollständig aufgenommen hatte und die darrunterliegenden bevorzugten Fließwege nicht angefärbt worden sind (BOUMA \& DEKKER, 1978). Im ungesättigten, ausgetrockneten Boden war die Adsorption des Farbstoffes an die Matrix außerordentlich hoch. Die Filterwirkung des Bodens gegenüber dem Farbstoff war also ebenfalls hoch. Dies zeigt, dass der Nachweis der bevorzugten Abflussbahnen mit Farbstoffen unter Feldbedingungen schwierig zu erbringen ist. Spätere Versuche, bei denen das Beregnungswasser direkt angefärbt worden war (BoumA et al., 1978), zeigten sowohl im ausgetrockneten, als auch im feldgesättigten Zustand eine tiefe Verlagerung des Farbstoffes in den Makroporen. Das Porenvolumen der für die gesättigte Wasserleitfähigkeit maßgeblichen Poren war unter $1 \%$ des Gesamtporenvolumens. Diese Poren hatten einen Äquivalentporendurchmesser von etwa 30 m (BOUMA \& BOOLTINK, 1990). Bei den genannten Ergebnissen handelt es sich um Auenböden und 
Marschböden in den südlichen Niederlanden. Es ist unklar, ob diese Ergebnisse auf die Pelosole übertragen werden können.

Die Verlagerung von fäkalen Mikroorganismen aus Gülle unter mitteleuropäischen Witterungsbedingungen auf schweren Böden betrachten die folgenden Arbeiten. MUNCH \& DOMINIK (1991) fanden auf einem Humusgley eine erheblich tiefere Verlagerung von Bakterien als auf einem Gley-Tschernosem und führten dies auf die Verlagerung in Schrumpfrissen zurück. Andere Arbeiten weisen keine oder nur eine geringe Verlagerung von fäkalen Mikroorganismen in strukturierten Böden mit Makroporen nach. Die Untersuchungen von WEIGEL (1995) zum Infiltrationsverhalten von Mikroorganismen in Böden ergaben auf dem tonigen Standort in den unterschiedlichen Tiefenabschnitten nur Keimzahlschwankungen im Bereich der natürlich vorkommenden Bodenmikroflora. Eine Verlagerung von fäkalen Mikroorganismen konnte von JOERGENSEN \& SEITZ (1998) auf einem unter Ackerbau befindlichen Röt-Pelosol nicht festgestellt werden. Grundsätzlich erschweren methodische Schwierigkeiten die Auswertung der Versuche, wie die ungleichmäßige Gülleausbringung und die Variation der Keimzahl in der Gülle bei Feldstudien (WAGNER, 1993).

Ähnlich wie Mikroorganismen werden auch manche Pflanzenschutzmittel als suspendierte Stoffe verlagert. HARIA et al. (1994) wiesen auf schweren Tonböden im Dränagewasser Pflanzenschutzmittel nach. Die Modellierung der Verlagerungsvorgänge bereitet jedoch weiterhin Schwierigkeiten (OOSTINDIE \& BRONSWIJK, 1995), da die Parametrisierung nicht genügend durch Felddaten abgesichert ist.

\subsubsection{Modellentwicklung für den Wasser- und Stofftransport in struktu- rierten Böden}

Die Modellierung des Solut- Transportes in strukturierten Böden haben BRUSSEAU \& RAO (1990) zusammengefasst.

Die Infiltration steht am Anfang der Wasserbewegung im Boden. Für die Überstauinfiltration gibt es ein Modell mit einer Ungenauigkeit von 1\% (BARRY et al., 1995). Überstaubedingungen kommen jedoch in der Natur so gut wie nie vor.

In der Vergangenheit sind Ein- und Mehr-Regionen-Modelle entwickelt worden, wobei aber das Ein-Regionen-Modell häufiger zur Anwendung kommt. BOHNE 
(1996) schreibt in einem Übersichtsartikel über Simulation des Wasser- und Stofftransportes, dass mehrregionale Strömungen in der Realität eine viel größere Bedeutung haben als ihnen weithin zugesprochen wird. Diese Strömungen sind komplizierter zu beschreiben und die Anzahl an Eingabedaten ist groß.

Das Dispersionsmodell führt im Felde jedoch zu einem Fehler der Massenbilanz von etwa 10\% (BOHNE, 1996). Das Problem bei der Simulation der Bodenfeuchte wird bei ausgetrockneten Tonböden darin gesehen, dass die Schrumpfrisse zuerst befeuchtet werden und später durch Quellung verschwinden. Das bedeutet, dass im gequollenem Zustand der Wasserfluss mit einem EinRegionen-Modell zu beschreiben wäre. RotH (1996) fordert, dass das Konzept der Homogenität unter isothermen Bedingungen des Porenraumes von Böden, welches allen Modellen im Labormaßstab unterlegt wird, in Zukunft fallen gelassen werden muss. Bohne (1996) zeigt auf, dass in der Zukunft Modellen ein deformierbares, mehrmodales Porensystem unter anisothermen Bedingungen zu Grunde liegen muss. Dabei sollen bevorzugte Wege und der Matrixtransport über zwei gekoppelte Richards-Gleichungen auf zwei nebeneinander vorkommende Porensysteme angewendet werden. Modelle mit bimodaler Porenraumverteilung entwickelten auch GERKE \& VAN GENUCHTEN (1993), BEVEN \& YOUNG (1988) und ZURMÜHL \& DURNER (1996). SCHLINDWEIN (1992) hat gezeigt, dass die Anwendung eines Zwei-Regionen-Modells eine erhebliche Verbesserung gegenüber dem Ein-Regionen-Modell in einem strukturiertem Boden gebracht hat.

Präferenzielle bzw. bevorzugte Flüsse können auch durch das Modell der kinematischen Welle beschrieben werden (GERMANN et al., 1987; LEVY \& GermanN, 1988; GermanN \& BürgI, 1996; Charbenau, 1984; SMith, 1983). Nach BoumA (1984) ist der "bypass flow" oder das "short circuiting" ein spezieller Fall des bevorzugten Wasserflusses. Dabei wird eine ungesättigte Matrix umgangen. In der Folge ist der Boden trotz Sickerwasserbildung nicht völlig aufgesättigt. Bevorzugte Wasserflüsse gibt es auch in wassergesättigten Systemen (WEBB \& ANDERSON, 1996). BOOLTINK (1994) befasste sich speziell mit dem „bypass flow” in einem schweren und stark texturierten Tonboden. Sowohl im trockenen Zustand, als auch nach Aufsättigung wurde "bypass" oder "preferential flow" nachgewiesen. Das bedeutet, dass Tonböden in jedem Feuchtigkeitszustand immer mit einem Mehr-Regionen-Modell zu beschreiben sind. 
Weiterhin sind Mehr-Regionen-Modelle entwickelt worden, die morphologische Verfahren mit den hydrologischen Messwerten kombinieren. Dabei kann auch die fraktale Dimension des Bodens berücksichtigt werden (DROOGERS et al., 1997; HATANO \& BOOLTINK, 1992) und eine Verbesserung der Modellanpassung erreicht werden.

Ein Problem vieler Untersuchungen ist nach BooLTINK (1994) die Vergleichbarkeit untereinander, oft fehlen wichtige Angaben zum Standort und der Veränderung des Quellungszustandes. Eine Übertragbarkeit auf andere Standorte ist deshalb nicht gegeben.

Für die Pelosole in Südniedersachsen ist es also fraglich, ob die genannten Ergebnisse übertragbar sind. Es bleiben einige Fragen offen, die näher untersucht werden sollen. Zu nennen sind die Variation des Porenraumes und die damit verbundenen Leitfähigkeitsänderungen, die letztlich den Stofftransport maßgeblich beeinflussen. 


\section{Beschreibung des Versuchsstandortes}

\subsection{Geographische Lage}

Das Untersuchungsgebiet liegt im Südniedersächsischen Bergland. Die mitteldeutsche Schwelle besteht im wesentlichen aus Schichten der Trias, die hauptsächlich in der Alpidischen Gebirgsbildung tektonisch beeinflusst worden sind (BRINKMANN, 1991). Dadurch sind weite Abschnitte der Mitteldeutschen

Schwelle als Schichtstufen- oder Bruchschollenlandschaft ausgeprägt.

Der Landschaftsaspekt der Rötlandschaft ist hügelig. An das Versuchsgebiet grenzt nach Westen hin der Kleine Knüll (von Mittelhochdeutsch Knollo bzw. Knoll = Erdschollen, Knolle, Hügel). Er liegt 294 m ü. NN. Die gleiche Bezeichnung wird im hessischen für die Basaltköpfe aus dem Tertiärvulkanismus verwendet, deren Oberflächenform ähnlich aussieht.

Der Versuchsstandort befindet sich auf einem Südhang mit 1-4\% Gefälle. Nach Norden schließt sich ein größeres Buchenmischwaldgebiet an, welches weitgehend auf Röt stockt. Die Versuchsfläche wurde bis 1991 als Ackerland genutzt und ist seitdem Dauergrünbrache, welche zeitweilig als extensives Weideland genutzt wird.

\subsection{Landschaftsformung und Prozesse des Periglazialraumes}

Die Geomorphologie der Mitteldeutschen Gebirgsschwelle ist während der Eiszeiten im wesentlichen durch den Einfluss des periglazialen Klimas im Zusammenhang mit der Eiszeit geprägt worden (ROHDENBURG, 1989). Entscheidend für das Südniedersächsische Bergland ist, wie auch für andere periglaziale Regionen, dass es hier nie ein direktes Überfahren der Landschaft mit Gletschereis gegeben hat. Im einzelnen sind die Prozesse der Frostverwitterung und der Solifluktion für unser Versuchsgebiet besonders prägend; deshalb soll hier gesondert darauf eingegangen werden. Eine Gesamtübersicht über die Prozesse im periglazialen Klima findet man bei SCHULTZ (1988), SMITH (1991), ROHDENBURG (1971), BLOOM (1998) und MüCKENHAUSEN (1985).

Durch Frostverwitterung, also im wesentlichen Frostsprengung, wurde das diagenetisch verfestigte Pelitgestein von der Bodenoberfläche her in kleine Pelitplättchen und schließlich bis hin zur Primärteilchengröße zerlegt. Makroskopisch verschwinden in diesem Prozess die Pelitplättchen und Steinchen, zurück 
bleibt nur Feinboden mit hohen Tongehalten. Deshalb wird dieser Prozess auch als Homogenisierung bezeichnet. Dieser homogenisierte Horizont bildet, wenn der Boden nicht umgelagert wird, den P-Horizont der Pelosole. Dies ist der Fall bei der autochthonen Bodenbildung. Nach DIEZ (1959) ist der Anteil der autochthonen gegenüber den allochthonen Pelosolen in Bayern nur sehr gering.

Die Umlagerung von Bodenmaterial im Periglazialklima kommt folgendermaßen zustande: In arktischen Klimaten ist der Boden in der Tiefe ganzjährig gefroren, man spricht von Permafrostboden. Während der sommerlichen Auftauphase kommt es dazu, dass die oberen Bodenschichten auftauen. Da die Verdunstung unter solchen Klimaten gering ist, und das über die Verdunstung hinaus fallende Niederschlagswasser durch den gefrorenen Boden nicht nach unten versickern kann, kommt es zur Sättigung oder sogar zur Übersättigung, der oberen Bodenschicht. Dabei entsteht Bodenbrei, der auch schon bei geringen Hangneigungen von 1-3 \% zu fließen beginnen kann. Dieses Fließen kann man sich nach MEYER (1999) wie die Bewegung eines Kartenspiels vorstellen, welches auf einem Tablett liegt und langsam durch das Kippen des Tabletts ins Rutschen gerät. Dabei rutschen die Spielkarten, die oben liegen durch die geringe Auflast am weitesten, während die unteren Karten sich nur ein wenig von der Stelle bewegen. Auf das Gelände übertragen bedeutet dies, dass die homogenisierten, an der Oberfläche befindlichen Partien am weitesten verlagert werden und das darunter befindliche Material nur einen kurzen Weg zurücklegt. Der Grundmechanismus der geschilderten Solifluktion durch Überschreiten der Fließgrenze wird als Frostbodenfließen bezeichnet. Der andere Grundmechanismus ist das Frostbodenkriechen, bei dem eine Abwärtsbewegung ausschließlich durch Frosthebung und Wiederabsenkung des Bodens beim sommerlichen Auftauen zustande kommt (SCHULTZ, 1988).

Der Solifluktionsschutt befindet sich oft am Hangfuß. Im Niedersächsischen Bergland ist er nach eigenen Beobachtungen durch Schmelzwasser oder später im Holozän erfolgte Erosion ausgeräumt worden. Bindige, tonige Substrate sind dabei besonders anfällig für Solifluktion, da sie im halbgefrorenen Zustand stark gleitfähig sind. Das Porenwasser ist gefroren, während das Adsorptionswasser in Abhängigkeit zur Nähe zu den Kolloidteilchen einen niedrigeren Gefrierpunkt hat. So ist im gefrorenen Boden noch Wasser vorhanden, welches den Boden gleitfähig hält. Durch die unterschiedlichen Fließgeschwindigkeiten und das 
Liegenbleiben der unteren Schichten kommt es dazu, dass die ursprüngliche oft leicht schräggestellte Gesteinslage an der Stelle, an der die Solifluktion beginnt und darüber, plötzlich in eine andere Richtung gestellt wird. Dies wird als Hakenschlagen nach BÜDEL (1937) bezeichnet. Solche Phänomene findet man im angrenzenden Wald im Norden des Versuchsgebietes. Dort gibt es auch Hinweise auf einige Erdfälle, also Dolinen, die durch Laugung des Gipses im Röt 1 entstanden sind. An das Hakenschlagen schließt sich dann laminare Solifluktion an, bei der die einzelne, farblich im ungestörten Pelitgestein, erkennbare Lage in einer wesentlich dünneren Solifluktionslage weiterverläuft.

Andere eiszeitliche, periglaziale Phänomene, die an anderen Orten im Göttinger Raum häufig anzutreffen sind, wie z.B. Eiskeile, Steinringe oder Strukturböden konnten bisher nicht festgestellt werden.

Der Röt als weicher Tonstein befindet sich zwischen zwei Festgesteinen, dem Mittleren Buntsandstein (sm, Sandstein) und dem Unteren Muschelkalk (mu, Kalkstein). Innerhalb der Landschaft bilden die Festgesteine die Steilhänge, während die weichen, wenig wasserdurchlässigen Pelitgesteine meist Terrassenflächen bilden.

Pelite sind weitgehend aus der Mittelgebirgslandschaft wie aus anderen Landschaften Deutschlands ausgeräumt worden. Das erklärt, dass der Flächenanteil von Pelosolen, bezogen auf das Bundesgebiet, nach BÜK 1:100.000 nur $5,45 \%$ beträgt. In der Schichtstufenlandschaft haben sich Pelite meist an Stellen gehalten, an denen sie durch Festgesteine überlagert sind. Im Versuchsgebiet ist diese Überlagerung nur an einigen Stellen erhalten, wie an den Köpfen des Eschenberges und des Knülls und lokal an noch einigen Stellen. Das betrachtete Rötgebiet ist also als Rumpffläche erhalten geblieben. Die Solifluktionserscheinungen sind auch für das folgende Kapitel von Bedeutung.

\subsection{Geologie und Röt-Stratigraphie}

Eine ausführliche Übersicht über die Feinstratigraphie des Oberen Buntsandsteins im südniedersächsischen Bergland gibt HINZE (1967). In Hessen haben WENZEL (1994) und DANZER (1964) die Stratigraphie des Röt dargestellt. Die Röt-Stratigraphie für Südniedersachsen ist nach HINZE (1967) in Tabelle 1 dargestellt. Eine feinstratigraphische Einordnung in eine der 4 (HINZE, 1967) oder 5 
(WENZEL, 1994) Untereinheiten ist nicht unproblematisch: Westlich vom Versuchsgebiet befindet sich der,Kleine Knüll“. Seine Spitze wird aus dem unteren Muschelkalk (mu) gebildet. Betrachtet man die angrenzenden, das Gebiet unterlagernden Schichten des mittleren Buntsandsteins (sm), so fällt auf, dass sich diese Schichten fast waagerecht auf dem Niveau von etwa 240-250 m ü. NN befinden. Die Mächtigkeit des Röt auf dem „Kleinen Knüll“ am Untersuchungsgebiet zwischen mittlerem Buntsandstein und Muschelkalk beträgt also nur etwa $50 \mathrm{~m}$. Die Mächtigkeit des Röt wird nach einer feinstratigraphischen Untersuchung im Niedersächsischen Bergland (HINZE, 1967) auf 120-140 m angegeben. Andere Untersuchungen aus dem benachbarten Nordhessen (WENZEL, 1994) geben eine Gesamtmächtigkeit von 104-115 m an.

Tabelle 1: $\quad$ Vereinfachte Röt-Stratigraphie für das Südniedersächsische Bergland nach HINZE (1967)

\begin{tabular}{lll}
\hline & Hauptmerkmale & Mächtigkeit \\
\hline Röt 4 & $\begin{array}{l}\text { vorwiegend violette, untergeordnet graue Tonsteine, in } \\
\text { geringem Maße hellgraue Feinsandsteinlagen }\end{array}$ & \\
& $48,3 \mathrm{~m}$ \\
\hline Röt 3 & braunroter Tonstein & $21 \mathrm{~m}$ \\
\hline Röt 2 & Wechsellagerung violetter und grüngrauer, teilweise & $25 \mathrm{~m}$ \\
& auch braunroter Tonsteine; bis zu 3 m mächtige Gips- \\
& steinlager & \\
\hline Röt 1 & $\begin{array}{l}\text { dunkelgraue und grüngraue Tonsteine mit mächtigen } \\
\text { Gipsstein- und Steinsalzlagern }\end{array}$ \\
\hline
\end{tabular}

Abbildung 2: Ausschnitt aus der Topographischen Karte 1:25.000 (Blätter 4525 Reinhausen und 4526 Gellihausen); (siehe nächste Seite) 


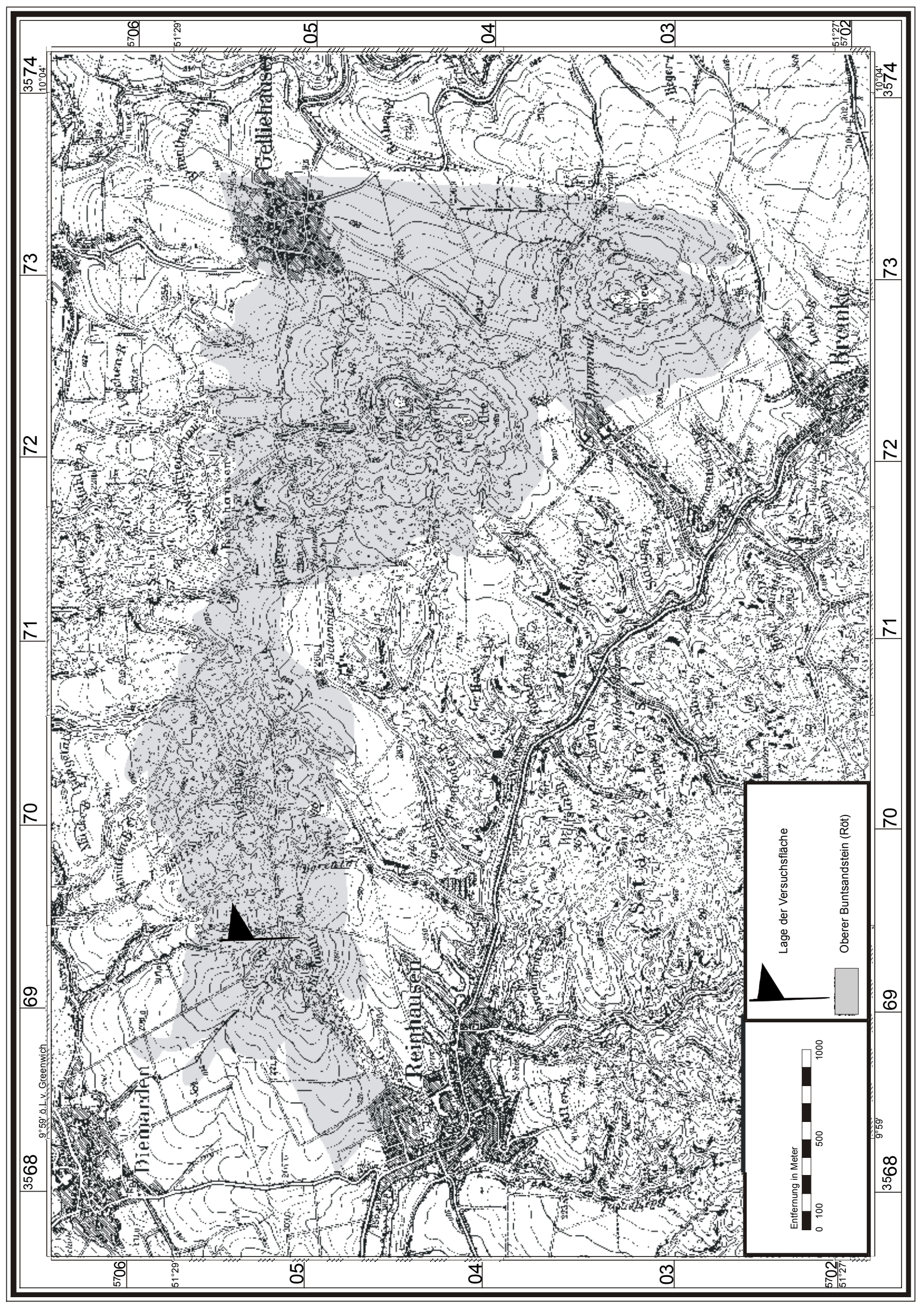


WENZEL (1994) berichtet von Massenverlagerungen und Rutschungen während der Eiszeiten. Aus den Geländebeobachtungen ist zu schließen, dass die sich nach Westen neigende Platte des Unteren Muschelkalkes vom Kleinen Knüll im Periglazial der letzten Kaltzeiten als großer Brocken auf den weichen Pelitschichten gerutscht und auf der Höhe des Röt 2 (40-65 m) liegengeblieben ist. WENZEL (1994) berichtet ähnliches aus dem nordhessischen Röt. Die ursprünglichen Schichten vom Röt 3 und 4 fehlen. Der Röt 1 (Salinarröt) unter dem Röt 2 enthält sehr reine Gipslinsen, die z.T. ausgelaugt sind. Dies erkennt man im nach Norden angrenzenden Waldgebiet an den vorhandenen Erdfällen unter denen sich Dolinen befinden. Für das Untersuchungsgebiet wird der Röt 2 angenommen, der nach Tabelle 1 als Wechsellagerung grauer und roter Tonsteine beschrieben wird, die im Gelände zu finden sind.

Die Rötverbreitung und die Lage des Versuchsgebietes sind in einem Ausschnitt der Topographischen Karte (Abbildung 2) eingetragen

\subsection{Die Böden aus Röt}

\subsubsection{Bodenentwicklung auf Röt}

Um die am Standort vorkommenden Böden besser einordnen zu können, betrachten wir kurz die Bodenentwicklungsreihe auf Tonsteinen (Tabelle 2): Grundsätzlich teilt man die Bodenentwicklungsreihen von Tonsteinen in kalkfreie und kalkhaltige Tonsteine ein. Die Bezeichnung ist Ton- oder TonmergelPelosol. Da das Ausgangsmaterial des Röt primär kalkhaltig ist, muss strenggenommen der Begriff Tonmergel-Pelosol verwendet werden. 


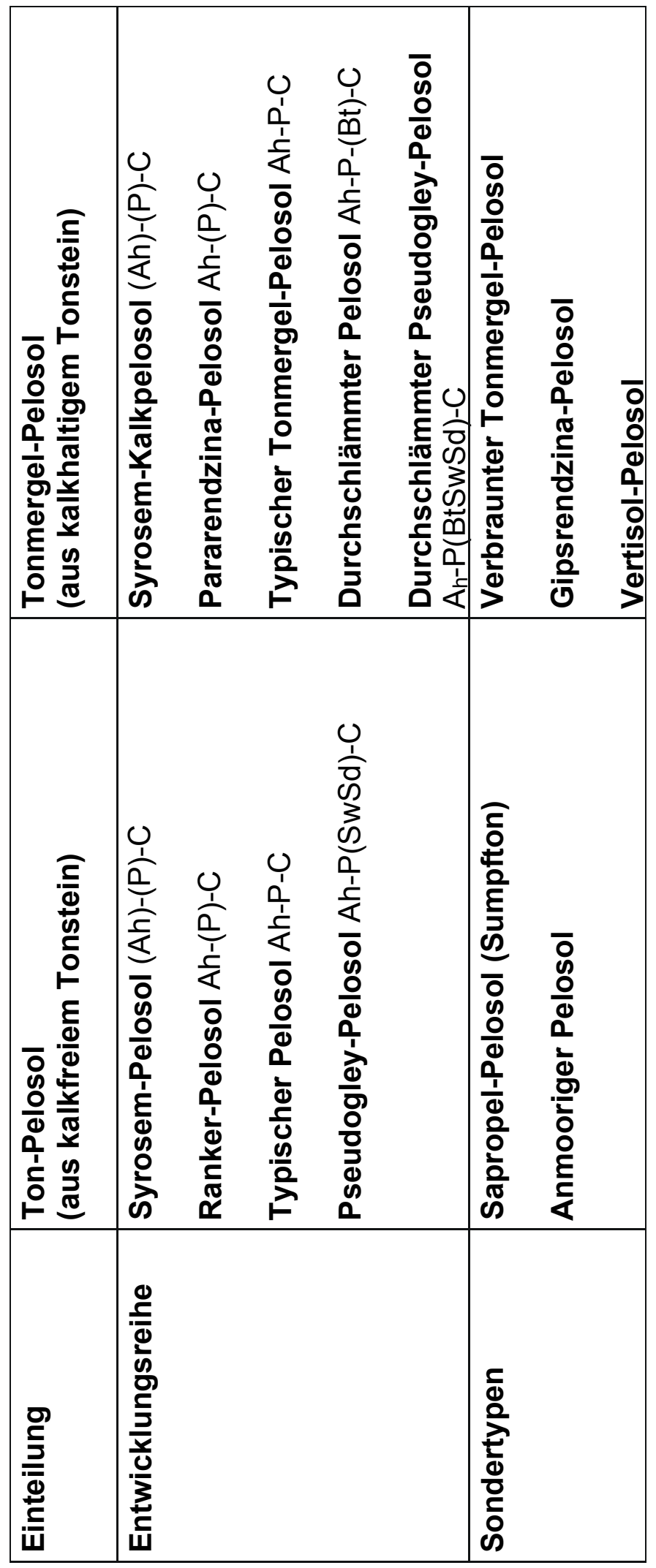

Tabelle 2: Einteilungsmöglichkeiten und Entwicklungsreihen der Böden auf Tonsteinen (Pelosolen) 
Die einzelnen Prozesse der Bodenentwicklung lassen sich folgendermaßen untergliedern: Zuerst bildet sich aus dem kalkhaltigen Substrat der SyrosemPelosol mit einer geringen Verwitterungsdecke. Im weiteren Verlauf der Bodenbildung entsteht der Pararendzina-Pelosol mit einem $A_{h}$-Horizont. Durch weiteren Zersatz des Pelitmaterials im Pleistozän und Holozän entsteht schließlich ein mächtiger, tonreicher Horizont, welcher eine hohe Gefügedynamik aufweist (GAESE, 1979). Aus Quellung und Schrumpfung resultiert ein grobes Prismengefüge, welches den P-Horizont bildet. In den obersten Dezimetern des Bodens wird gleichzeitig Humus akkumuliert. Deshalb wird dieser Horizont oft als Ah bezeichnet, obwohl er, wie auch die darunterliegende Schicht zum P-Horizont gehört. In der weiteren Bodenentwicklung kommt es zur Tonverlagerung aus dem Oberboden. Dieser Ton setzt sich in darunterliegenden Schichten als Überzug auf den Bodenaggregaten ab. Es treten sogenannte Tontapeten auf, dies ist dann ein durchschlämmter Pelosol. Die weitere Verschlämmung und Verdichtung der Aggregate durch Quellung und Schrumpfung geht so weit, dass es zu Stauwasser und schließlich zu Pseudogleymerkmalen kommt. Dieser Zustand ist unter unseren Klimabedingungen der Endzustand der Bodenentwicklung, Vertisolvorkommen sind in Südniedersachsen unbekannt.

Bei kalkhaltigen Ausgangssubstrat entsteht so der Kalk-Pelosol. Am Standort sind die Aggregate des P-Horizontes meist schon vollständig entkalkt. Hier spricht man vom Typischen Pelosol oder nach AG BoDEN (1994) vom NormPelosol.

\subsubsection{Beschreibung der Böden am Versuchsstandort}

Der vorherrschende Bodentyp ist der Pelosol aus Röt. Eine vorliegende Korngrößenanalyse (MEIWES, 1974) im Ap gibt folgende Informationen: 17 \%S, $30,7 \%$ U, 52,3\% T, das entspricht nach bodenkundlicher Kartieranleitung (AG BODEN, 1994) schwach schluffiger Ton (Tu2). Die Reichsbodenschätzung stuft den Boden am Versuchstandort als Verwitterungsboden ein (T 4 V 49/44 bzw. T 5 V 44/39). Dies kann im Gelände anhand einiger Bohrungen nachvollzogen werden, weil sich an einigen Stellen Fließerden bzw. Solifluktionslagen mit teilweise noch unverwitterte Pelitplättchen aus dem Ausgangsmaterial befinden. Die Wechsellagerung von mehr oder weniger verwitterten Schichten setzt sich 
stellenweise bis in Bodentiefen von über $2 \mathrm{~m}$ fort. Stellenweise gibt es LössInseln, in denen dann der verbraunte Pelosol vorkommt.

Tabelle 3: Zusammengefasste Merkmale einiger Pelosol-Bodenprofile am Versuchsstandort in Reinhausen am „Kleinen Knüll“; bei den Bodentiefen sind jeweils die Spannbreiten der Horizonte angegeben

\begin{tabular}{|c|c|c|}
\hline $\begin{array}{l}\text { Horizont, Bodentiefe } \\
\text { (cm) }\end{array}$ & Merkmale & Substrat \\
\hline $\begin{array}{l}\text { Ah (p ehemals) }(P) \\
0-20 \mathrm{~cm}\end{array}$ & $\begin{array}{l}\text { Polyedergefüge }(3-4 \mathrm{~cm}) \text { wenige ver- } \\
\text { tikale Klüfte im feuchten Zustand } \\
\text { sichtbar }\end{array}$ & Rötton \\
\hline $\begin{array}{l}P \\
20-40 \mathrm{~cm} \\
20-60 \mathrm{~cm}\end{array}$ & $\begin{array}{l}\text { Prismengefüge, in Polyeder zerleg- } \\
\text { bar, vertikale Klüfte dominieren, stel- } \\
\text { lenweise Manganflecken; Aggregat- } \\
\text { oberflächen speckig glänzend, Ton- } \\
\text { tapeten }\end{array}$ & Rötton \\
\hline $\begin{array}{l}\text { PCv } \\
40-60 \mathrm{~cm} \\
60-80 \mathrm{~cm}\end{array}$ & $\begin{array}{l}\text { Übergang des P-Horizontes in eine } \\
\text { eher plattige Gesteinsstruktur }\end{array}$ & $\begin{array}{l}\text { Rötton (mit So- } \\
\text { lifluktionslage) }\end{array}$ \\
\hline $\begin{array}{l}\text { Cv } \\
60-80 \mathrm{~cm} \\
80-100 \mathrm{~cm}\end{array}$ & Pelitgesteinszersatz & Rötton \\
\hline $\begin{array}{l}\text { Cv } \\
80-100 \mathrm{~cm} \\
100-120 \mathrm{~cm}\end{array}$ & $\begin{array}{l}\text { plattige Struktur, waagerecht ausge- } \\
\text { richtet }\end{array}$ & $\begin{array}{l}\text { Grün-graue } \\
\text { feinplattige Röt- } \\
\text { lage mit Glim- } \\
\text { mern auf der O- } \\
\text { berfläche }\end{array}$ \\
\hline $\begin{array}{l}\text { Cv } \\
>100-120 \mathrm{~cm}\end{array}$ & Pelitgestein & Rötton \\
\hline
\end{tabular}

Ein typisches Bodenprofil am Versuchsstandort (Tabelle 3) hat eine Solifluktionslage aus braunem und grauem Material. Das Profil ist ein Ah-P-Cv Profil, wobei der Ah- und der P-Horizont ein ausgeprägtes Polyedergefüge mit starker Quellungs- und Schrumpfungsdynamik enthält. Die Polyeder sind bis in größere Bodentiefen (>80 cm) mit Tonüberzügen aus dem Ah versehen. Solche Überzüge entstehen (DIEZ, 1959), wenn Wasser bei trockenem Boden in Schrumpfrisse infiltriert. Dabei wird Ton aus dem Oberboden mitgeführt. Weiter unten wird das Wasser von den Aggregaten aufgenommen oder die Fließgeschwindigkeiten sind so gering, dass der Ton sich an den Aggregaten anlagert. Es kommt zur Bildung von sogenannten Tonlippen und -rippen. Wann dieser Prozess abgelaufen ist, und ob er noch rezent ist, kann hier nicht festgestellt wer- 
den. Stellenweise treten Pseudogleymerkmale auf, welche auf eine geringe Luftkapazität schließen lassen. Dabei fällt weniger die auftretende Bleichung, als vielmehr die Manganflecken dem Betrachter ins Auge.

Vertisoleigenschaften fehlen, die Tonüberzüge sind zwar stark verdichtet, jedoch existieren keine Slickensides. Die Bezeichnung Vertisol-Pelosol scheidet also aus. Tonüberzüge, Verbraunungs- und Pseudogleymerkmale treten nicht überall auf, deshalb sind sie nicht in die Horizontierung aufgenommen worden. Hier handelt es sich also um einen Typischen Pelosol oder Norm-Pelosol, nach FAO-Systematik (FoOd AND AGRICULTURE ORGANIZATION OF THE UNITED NATIONS, 1990) um einen Eutric-Vertisol mit Stagni-Eutric Vertisol Merkmalen. 


\section{Feldmessungen}

\subsection{Untersuchungen zum Wasserhaushalt und zur Wasserbe- wegung}

\subsubsection{Methodik}

\subsubsection{Arbeitsziele}

Das erste Ziel ist es, die Wasserhaushaltsbilanz nach DVWK (1980) für ein Normaljahr aufzustellen:

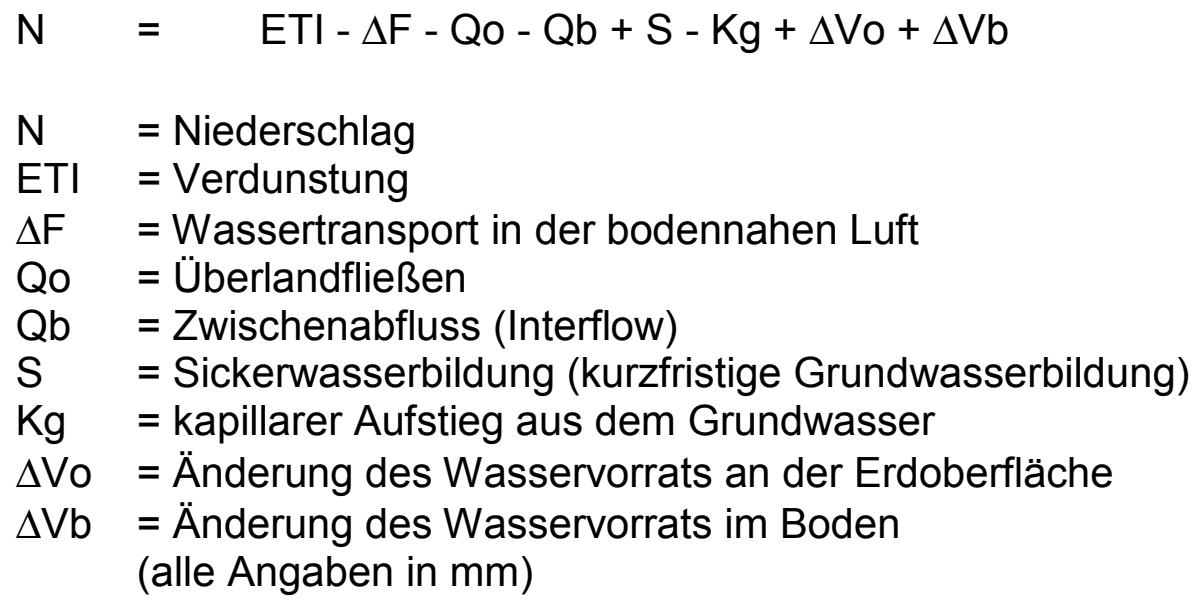

Die Wasserhaushaltsgleichung wird für viele Fälle vereinfacht: Oberirdischer Abfluss sollte durch entsprechende Maßnahmen bei der Bewirtschaftung vermieden werden. In ebenen Lagen ist Zwischenabfluss (Interflow) in der ungesättigten Zone auszuschließen. Mit den Lysimetern soll die Sickerwasserspende gemessen werden. Kapillarer Aufstieg wird wegen der niedrigen texturbedingten hydraulischen Leitfähigkeit vernachlässigt. Die Vorratsänderung wird nur im gesamten Boden betrachtet $(\Delta \mathrm{V})$ :

$$
\begin{array}{ll}
\mathrm{N} & =\quad \mathrm{ETI}+\mathrm{S} \pm \Delta \mathrm{V} \\
\mathrm{N} & =\text { Niederschlag [mm] } \\
\mathrm{ETI} & =\text { Verdunstung [mm] } \\
\Delta \mathrm{V} & =\text { Änderung des Bodenwasservorrats [mm] } \\
& \text { (alle Angaben in } \mathrm{mm} \text { ) }
\end{array}
$$

Das zweite Ziel dieser Untersuchungen ist, die maximal ausschöpfbare Feldkapazität im effektivem Wurzelraum (nFkWe) zu ermitteln. Es ist ein Eingabeparameter für viele Wasserhaushaltsmodelle. Dieser Wert gibt den Anteil des Bodenwasservorrats bei Feldkapazität an, der den Pflanzen zur Verfügung steht. Bei Annahme des Ein-Region-Modells geht man dann davon aus, dass dieser 
Vorratswert bei positiver Niederschlagsbilanz wieder aufgefüllt werden muss, bevor es zur Sickerwasserbildung kommt. Mit Lysimetern und begleitenden Bodenfeuchtemessungen soll der Zeitpunkt der Aufsättigung des Bodens bestimmt werden. Zur Bewertung von nFkWe und der maximalen Feldkapazität ist eine Lysimetermethode zu wählen, die die Messung der Tiefeninfiltration des Niederschlagswassers ermöglicht.

Ein weiteres Ziel ist die Messung des Stoffaustrages. Mit Hilfe von Tracern soll das Retentionsverhalten für gelöste Stoffe bei verschiedenen Quellungszuständen untersucht werden. Untersuchungen zum wassergetragenen Partikeltransport sind zur Klärung des Keim- und Pflanzenschutzmitteltransportes geplant.

\subsubsection{Methodenwahl zur Messung der Wasserbewegung im Freiland} Lysimeter werden zur Messung von wassergetragenen Stoffflüssen in Böden verwendet. Einen umfassenden Überblick über Einsatzmöglichkeiten von Lysimetern geben DVWK (1980) und TITUS \& MAHENDRAPPA (1996). Das Lysimeter klassischer Bauart, wie das Lysimeter nach FRIEDRICH \& FRANZEN (1960), ist ein großes Gefäß, welches mit Bodenmaterial gefüllt ist und geeignet ist, das Wasser aufzufangen, welches an der Unterseite den Boden verlässt. Wenn das Lysimeter tief genug ist, und bis in die Dränzone des Bodens reicht, in der nur noch abwärts gerichtete Bodenwasserbewegung stattfindet, so ist das aufgefangene Wasser das Sickerwasser. In Verbindung mit der Messung der Niederschläge ist so die Erstellung einer Bodenwasserbilanz bzw. einer vereinfachten Wasserhaushaltsgleichung möglich, die in Abschnitt 4.1.1.1 erwähnt wurde.

TITUS \& MAHENDRAPPA (1996) teilen Lysimeter in vier Kategorien ein, abhängig von der Begrenzung des Bodens, der Störung des Bodens, der Wägeeinrichtung und des Tensionstypes.

Die Begrenzung des Bodens betrifft besonders die seitliche Begrenzung des Bodenkörpers. In diesem Zusammenhang wurden in der Literatur vielfach die seitlichen Randeffekte diskutiert. Durch eine seitliche Lysimeterbegrenzung werden die Pflanzenwurzeln an ihrem natürlichen Wachstum gehindert. Es kommt zu einer großen Konzentration von Pflanzenwurzeln an der Lysimeterwand, die auch Auswirkungen auf die Hydrologie hat (DVWK, 1980; SCHIFF, 1971; HOYNIGEN-HUENE \& BRAMM, 1978). Laterale Flüsse werden durch eine seitliche Lysimeterwand ausgeschlossen. Weiterhin gibt es Hinweise auf die 
Veränderung der Bodentemperatur und des oberirdischen Klimas durch die Lysimeterwand (ROTH et al., 1989; SCHRÖDTER, 1985; HOYINGEN-HUENE \& BRAMM, 1978). Der Kontakt zur Lysimeterwand ist schlecht herzustellen, deshalb kann die Wand indirekt als Dränung wirken (OLBERTZ, 1957).

Die Störung des Bodens wird im Wesentlichen durch die Art der Befüllung verursacht. Gut sind monolithische Lysimeter, die einen natürlich gelagerten und ungestörten Boden enthalten. In vielen Fällen ist jedoch aufgrund der Größe der Lysimeter die monolithische Befüllung zu aufwendig. In solchen Fällen wird das Bodenmaterial mit dem Spaten oder mit einem Bagger in den Lysimeterbehälter eingefüllt. Dabei geht die natürliche Bodenstruktur verloren. WEINZIERL (1984) beobachtete eine stärkere Ausschöpfung des Wasservorrats in künstlich befüllten Löss-Lysimetern gegenüber einer natürlich gelagerten $\mathrm{Pa}$ rabraunerde aus Löss. Für die Sickerwasserbildung bedeutet dies, dass der Zeitpunkt der ersten Sickerwasserbildung später stattfindet und dass in der Summe weniger Sickerwasser gebildet wird. Die Übertragbarkeit der Daten aus gestörten Lysimetern in das Freiland ist also nur eingeschränkt möglich. Die Veränderung der Porengrößenverteilung des Bodens ist für hydrologische Messungen nicht zu akzeptieren. Es kommen also nur monolithische Lysimeter in Frage.

Die Einteilung in nicht wägbare und wägbare Lysimeter hat grundsätzlich nur etwas mit dem Einsatzzweck der Lysimeter zu tun. Wägbare Lysimeter werden für eine hohe zeitliche Auflösung der Messwerte von Wasserhaushaltskomponenten genutzt. Mit den gemessenen Niederschlägen und mit der Wägung der Ausschöpfung des Bodenwasservorrats kann die Verdunstung bestimmt werden. Die kann, in Verbindung mit dem Ausschöpfungsgrad der Feldkapazität, in der Beregnungsberatung eingesetzt werden. Darüber hinaus ist es möglich, die nFkWe, also die maximal mögliche Beanspruchung der Feldkapazität, zu bestimmen, wie sie bei dem Verdunstungsmodell von SPONAGEL (1980) eingesetzt wird.

Das letztgenannte Unterscheidungskriterium ist der Tensionstyp des Lysimeters. Die Störung des natürlichen Saugspannungsprofils ist nach OLBERTZ (1957) der "Kardinalfehler" der Lysimetermethode. Grundsätzlich ist mit einem Lysimeter die Begrenzung des Bodens nach unten verbunden. Die natürliche 
Verbindung zum Grundwasser wird unterbunden. In einem Lysimeterbehälter mit einem freien Abfluss an der Unterseite kann das Wasser nur ungespannt den Boden verlassen. Unter natürlichen Bedingungen wäre in der entsprechenden Tiefe noch eine negative Saugspannung vorhanden. Daraus resultiert eine für die Pflanze bessere Wasserverfügbarkeit als im Freiland. Darüber hinaus kann es zum Einstau von ungespanntem Wasser im unteren Lysimeterbereich kommen (SieBNER, 1995). Dadurch kann es zu kapillarem Aufstieg im Boden und so zu einem zusätzlichen Wasserangebot gegenüber den Freilandbedingungen kommen. Andererseits wird auch der kapillare Aufstieg aus Bodenschichten unterhalb des Lysimeterbodens verhindert. Bei der Übertragung von Lysimeterdaten in das Freiland müssen also immer die Unterschiede zwischen den hydrologischen Eigenschaften des speziellen Lysimetertyps und den natürlichen Bodenbedingungen berücksichtigt werden. Grundsätzlich wird in vielen Fällen das Wasserangebot infolge niedrigerer Saugspannung und zusätzlichem Vorrat an ungespanntem Wasser das gesamte Wasserangebot in den Lysimetern gegenüber dem Freiland erhöht. Dadurch kommt es zum sogenannten Oaseneffekt (SCHROEDTER, 1985). Dieser hat zur Folge, dass die Pflanzen im Lysimeter eine überproportional hohe Verdunstung haben, da das Sättigungsdefizit der Luft durch die benachbarten Pflanzen, welche weniger Wasser zur Verfügung haben, höher ist, als es bei der eigentlichen Bodenfeuchte im Lysimeter der Fall wäre. Neben den drucklos betriebenen Lysimetern gibt es auch Unterdrucklysimeter. Bei den Unterdrucklysimetern wird an der Unterseite des Bodens eine Saugspannung angelegt, die möglichst der Saugspannung im natürlichen Boden entsprechen soll. Der technische Aufwand hierfür ist sehr groß. Theoretisch gesehen können so jedoch am ehesten die natürlichen Bodenverhältnisse simuliert werden.

In klassischen Lysimetern mit seitlicher Begrenzung können Randeffekte nicht vermieden werden. Das Kontakthalten zur Lysimeterwand bereitet besonders in Böden mit starker Quellungs- und Schrumpfungsdynamik Schwierigkeiten. Außerdem haben die Pflanzenwurzeln an der Lysimeterwand eine höhere Dichte als im ungestörten Boden (BÖHM, 1979) und hinterlassen nach dem Absterben Poren, in denen dann das Wasser bevorzugt fließen kann. Durch das Schrumpfen und Quellen des Bodens kann der Kontakt mit der Lysimeterwand 
nicht gewährleistet werden. Der verbleibende Raum zwischen Wand und Boden kann als zusätzliche Dränung wirken. Bevorzugtes Fließen ist die Folge.

\subsubsection{Modifizierung der Lysimetermethode für Freilandmessungen in Pelosolen}

Bei der Auswahl eines für die Versuchsfrage geeigneten Lysimetertyps ist es notwendig, das hydrologische Verhalten des Versuchsbodens zu berücksichtigen. Aufgrund des Quellungs- und Schrumpfungsverhaltens des Röt-Pelosols ist damit zu rechnen, dass im aufgesättigten, gequollenem Zustand kaum nennenswerte Wasserbewegungen existieren, da nur die Texturporen wirksam sind. Mit zunehmender Austrocknung treten vermehrt Schrumpfrisse als Makroporen auf. Eine fast vollständige Entwässerung solcher Poren ist aufgrund des großen Äquivalentporendurchmessers ohne zusätzlich angelegte Saugspannung möglich. Da die Wasserbewegung in den texturbedingten Poren nur sehr gering ist im Vergleich zu den Makroporenflüssen, kann die Messung der Matrixporenflüsse aus zwei Gründen vernachlässigt werden: Zum einen zählt die Matrix nach der Modellvorstellung von BECHER (1985) nur dann zur mobilen Phase, wenn im Boden ein Potentialgradient anliegt. Zum anderen besteht die Matrix aus großen Aggregaten, es handelt sich also nur um Bewegungen innerhalb der Aggregate, also nur um Bewegungen in sehr begrenztem Umfang. Der maßgebliche Teil der Wasserbewegung kann also durch Messung der Makroporenflüsse erfolgen.

Um die Randeffekte zu umgehen und das ungespannte Wasser zu erfassen, wurden die bei JÖRGENSEN (1987) beschriebenen Plattenlysimeter benutzt. Es handelte sich um 0,3 $\mathrm{m}^{2}$ große PVC-Platten, die unter den ungestörten Boden geschoben wurden, um somit das frei dränende Wasser aufzufangen. Diese wurden aus einem Graben (Abbildung 3 ) heraus in 30, 40, 50, 60, 70, 80, und $90 \mathrm{~cm}$ Bodentiefe installiert. Damit wurde gewährleistet, dass die Tiefeninfiltration erfasst werden konnte. In dem Graben wurde ein Lysimeterkeller eingerichtet, in dem das Wasser aus den einzelnen Lysimetern in Polyethylen-Kanistern aufgefangen wurde. Die Wände waren aus Holz und gegen die Bodenfeuchte mit Folie abgedichtet.

Die Niederschläge wurden in einem Hellmann-Niederschlagsmesser in $1 \mathrm{~m}$ Höhe gemessen. Um ackerbauliche Nutzung zu simulieren wurde im Oktober 1996 
eine Fläche von $30 \mathrm{~m}^{2}(3 \cdot 10 \mathrm{~m})$ mit dem Spaten etwa $25 \mathrm{~cm}$ tief umgegraben. Diese Fläche wird im folgenden als Ackerfläche bezeichnet. Im Frühjahr 1997 wurde die gesamte Ackerfläche zeitweilig mit einer Gießkanne mit einem fein verteilendem Brausekopf bewässert. Zeitweilig wurde die gesamte Ackerfläche mit einer Plane bedeckt, um Verdunstung und Niederschläge vom Boden während einer Beregnungsphase fernzuhalten.

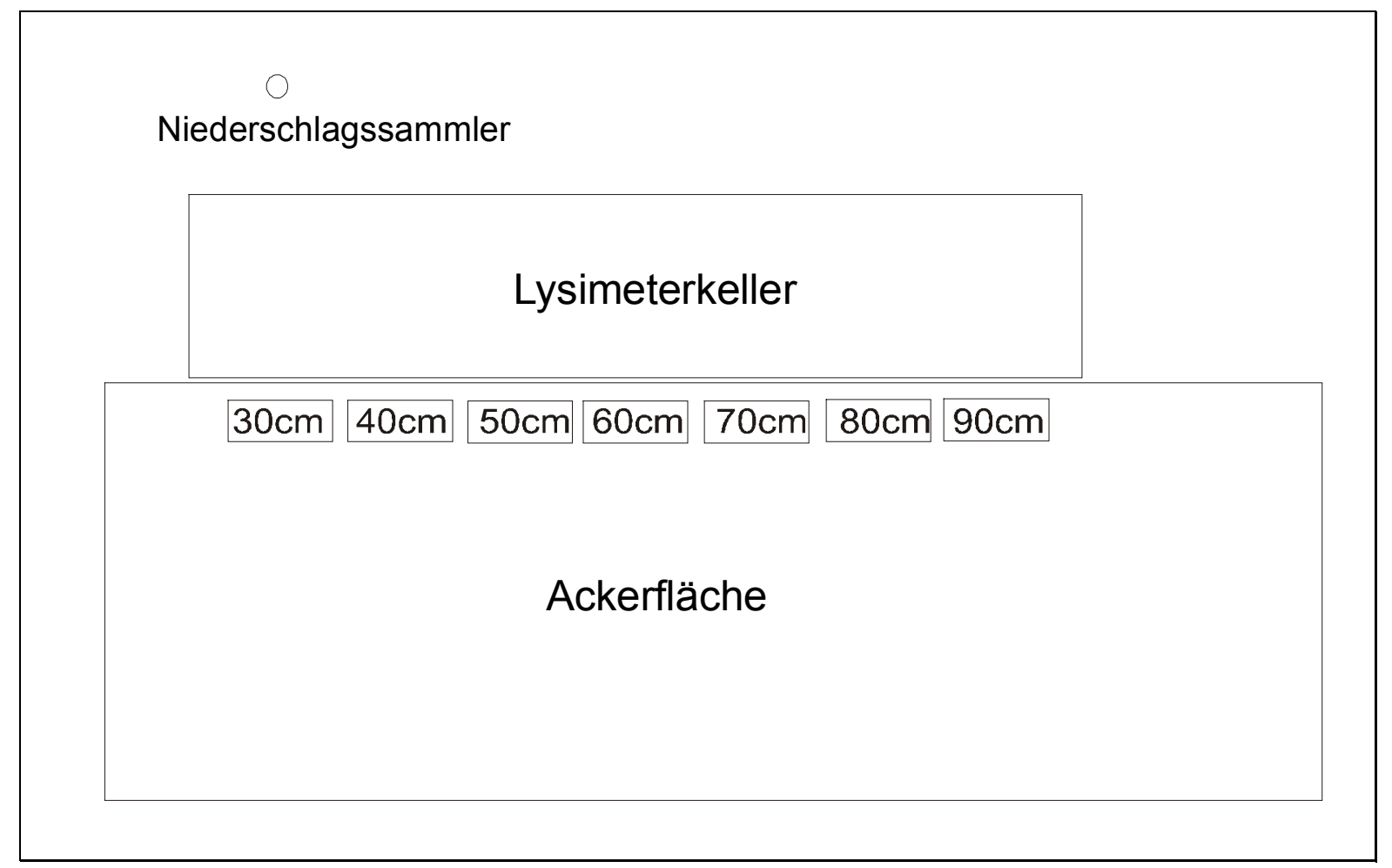

Abbildung 3: Anordnung der Plattenlysimeteranlage am Kleinen Knüll; Breite der Ackerfläche 10 m; Norden ist oben, weitere Erläuterung im Text

Die Lage der Lysimeteranlage wurde so gewählt, dass es zum Lysimeterkeller und damit zu den Lysimetern leicht ansteigt. So sollte zusätzliches InterflowWasser in den Lysimetern vermieden werden.

Im zeitigen Frühjahr wurden die Wassergehalte des Bodens bestimmt. Zusammen mit den Wassergehalten im Sommer im ausgetrockneten Zustand bekommt man die nutzbare Feldkapazität im effektiven Wurzelraum. Die Wassergehalte müssen deshalb als Volumenprozente vorliegen. Zu diesem Zweck wurde parallel zu den Bohrungen ein Tiefenprofil mit einem Wurzelbohrer er- 
stellt, welches ein definiertes Volumen hat. Der gemessene Wassergehalt wird dann auf das definierte Bodenvolumen bezogen.

Für die meisten Böden kann man für die Festsubstanzdichte von Quarz $\left(2,65 \mathrm{~g} \mathrm{~cm}^{-3}\right)$ einsetzen (HARTGE \& HORN, 1989). Mit deren Hilfe man dann die Trockendichte $\left[\mathrm{g}^{\mathrm{cm}}{ }^{-3}\right]$ errechnet. Ist Festsubstanzdichte bekannt, so kann man die Porenziffer $\left(\varepsilon_{P}\right)$ ableiten:

$$
\varepsilon_{P}=\frac{d_{B}}{d_{F}} \cdot 100[\%]
$$

Dieser Parameter wird in der Bodenmechanik verwendet, wenn man Gefügeveränderungen, wie z. B. Lockerung oder Verdichtung beurteilt. Wie in der Bodenmechanik kann man die für die Wasseraufnahme des Bodens eine Wasserziffer $\left(\varepsilon_{w}\right)$ definieren:

$$
\varepsilon_{w}=\frac{V_{w}}{V_{\text {fest }}} \cdot 100[\%]
$$

Die Wasserziffer $\left(\varepsilon_{\mathrm{w}}\right)$ ist der Quotient aus dem wassergefüllten Porenvolumen $\left(V_{w}\right)$ und dem Volumen der Festsubstanz $\left(V_{\text {fest }}\right)$. Der Bezug auf die Festsubstanz bringt Aufschlüsse, wie sich Veränderungen der Aggregierung im Profil auf die Wasserspeicherung einzelner Bodenschichten auswirken. Es gibt das Verhältnis an, wie viel Gramm Wasser ein Gramm Boden speichern kann.

\subsubsection{Ergebnisse und Diskussion}

In Tabelle 4 sind die Niederschlagssummen und Sickerwassersummen vom 1.10. 1996 bis zum ersten Schneefall aufgetragen. Im September 1996 hatte es an der Niederschlagsmessstation am Institut für Bodenwissenschaften in Göttingen 25,9 mm geregnet. Das liegt weit unter dem Jahresmittelwert von $48 \mathrm{~mm}$ für Göttingen für den Monat September im langjährigen Mittel (SCHIRMER \& VENT-SCHMIDT, 1979). Es wird deshalb davon ausgegangen, dass die Böden beim Einbau der Lysimeteranlage Ende September stark ausgetrocknet waren. Hierfür sprechen die im Gelände festgestellte Schrumpfrisse bis in $90 \mathrm{~cm}$ Bodentiefe. 
Tabelle 4: $\quad$ Summen von Niederschlag $(N)$ und Sickerwasser $(S)$ aus tiefengestaffelten Plattenlysimetern in 30-90 cm Bodentiefe in $\mathrm{mm}$ nach der sommerlichen Austrocknung (Messbeginn: 1.10.96)

\begin{tabular}{lrrrrrrrr}
\hline Datum & N & S 30 & S 40 & S 50 & S 60 & S 70 & S 80 & S 90 \\
\hline 18.10 .96 & 45,3 & 24,8 & 13,8 & 16,7 & 17,9 & 39,3 & 33,3 & 44,6 \\
04.11 .96 & 27,1 & 26,6 & 10,1 & 8,8 & 11,0 & 39,0 & 42,9 & 41,7 \\
22.11 .96 & 20,1 & 4,4 & 16,0 & 24,7 & 24,9 & 36,3 & 40,7 & 45,3 \\
09.12 .96 & 55,9 & 4,5 & 14,1 & 22,1 & 24,9 & 69,9 & 64,7 & 55,3 \\
\hline Summe & 148,4 & 60,3 & 54,0 & 72,3 & 78,7 & 184,6 & 181,7 & 186,8 \\
\hline $\begin{array}{l}\text { \%S von } \\
\text { N }\end{array}$ & & 40,6 & 36,4 & 48,7 & 53,0 & 124,4 & 122,4 & 125,9 \\
\hline
\end{tabular}

Die Sickerung begann sofort mit einsetzenden Niederschlägen, also vor Auffüllen des Bodenwasservorrates. Daraus folgt, dass die Vorstellung fallen gelassen werden muss, mit einem Verdrängungsmodell (BÖTTCHER et al., 1983) arbeiten zu können, bei dem der ausgeschöpfte Bodenwasservorrat aufgefüllt wird und erst anschließend die Sickerung einsetzt. Im Zeitraum vom 1.10. bis zum 31.12.1996 fielen an der Messstation am Institut für Bodenwissenschaft maximal 4,2 $\mathrm{mm} / \mathrm{h}$, im Median nur 0,3 $\mathrm{mm} / \mathrm{h}$. Zwar können diese Werte streng genommen nicht nach Reinhausen übertragen werden, jedoch stützen sie die eigene Beobachtung, dass in diesem Zeitraum keine Starkniederschlagsereignisse vorgekommen sind. Nach WEISCHET (1995) spricht man von Starkregen erst ab einer Intensität von $5 \mathrm{~mm}$ in 5 Minuten oder von $16 \mathrm{~mm}$ in einer Stunde. Bei diesen Niederschlagsintensitäten erfolgt dennoch eine Infiltration bis in $90 \mathrm{~cm}$ Bodentiefe. Auch ohne die Niederschlagsintensität eines Starkregenereignisses kommen demzufolge präferenzielle Flüsse vor.

Weiterhin ist zu folgern, dass die Wasserflüsse die teilweise noch ungesättigte Matrix des Bodens umgehen. Nach BoumA et al. (1981) werden solche Flüsse als „short circuiting“, später (BoumA, 1984) als „bypass flow“ bezeichnet. Der „bypass flow" ist also ein Sonderfall des „preferential flow", der auch in wassergesättigten porösen Medien vorkommen kann (WEBB \& ANDERSON, 1996). 
BoOLTINK (1995) beobachtete 84 \% der winterlichen Niederschläge im Dränagenwasser. Begleitende Untersuchungen zeigten hier, dass die Feldkapazität erst zu einem späterem Zeitpunkt erreicht wurde.

Tabelle 5: $\quad$ Monatssummen von Niederschlag $(N)$, Beregnung $(B)$ und Sickerwasser (S) aus tiefengestaffelten Plattenlysimetern in 30$90 \mathrm{~cm}$ Bodentiefe in $\mathrm{mm}$

\begin{tabular}{lrrrrrrrr}
\hline Monat & N + B & S 30 & S 40 & S 50 & S 60 & S 70 & S 80 & S 90 \\
\hline Oktober & 67,5 & 51,1 & 23,8 & 25,5 & 28,2 & 39,3 & 33,3 & 45,2 \\
November & 55,9 & 5,1 & 16,4 & 24,7 & 26,6 & 37,2 & 41,8 & 50,2 \\
Dezember & 25,0 & 41,9 & 21,9 & 29,3 & 56,3 & 105,1 & 94,5 & 86,6 \\
Januar & 0,0 & 4,2 & 0,0 & 0,4 & 2,2 & 5,1 & 1,7 & 2,4 \\
Februar & 76,4 & 39,8 & 33,5 & 47,9 & 67,5 & 63,2 & 91,8 & 101,0 \\
März & 102,4 & 30,2 & 32,6 & 33,9 & 77,3 & 79,0 & 113,6 & 86,4 \\
April & 85,1 & 27,8 & 22,0 & 16,4 & 46,0 & 35,5 & 41,5 & 26,4 \\
\hline Summe & 412,3 & 200,1 & 150,1 & 178,1 & 304,0 & 364,4 & 418,2 & 398,2
\end{tabular}

In der Summe liegen die Wasserbilanzen der Lysimeter in 30 bis $60 \mathrm{~cm}$ Bodentiefe unter $100 \%$. Dies ist als Folge der Evapotranspiration zu erwarten. Die Lysimeter in 70 bis $90 \mathrm{~cm}$ Bodentiefe haben in der Summe über $100 \%$ Sickerwassermenge gegenüber dem gefallenden Niederschlag. Daraus folgt, dass unklar ist, wie groß die Einzugsgebiete der einzelnen Lysimeter sind. Naheliegend ist, dass die Einzugsgebiete der Lysimeter größer sind als ihre eigentliche Oberfläche von $0,3 \mathrm{~m}^{2}$.

Tabelle 5 zeigt die Monatssummen der Tiefeninfiltration in den einzelnen Bodentiefen im Winterhalbjahr und April 1996 / 97. Zu keinem Zeitpunkt kommt die Tiefeninfiltration zum Erliegen. Die Annahme, dass die Makroporen beim Quellen des Bodens verschwinden, muss fallen gelassen werden, sonst gäbe es wegen der geringen gesättigten Leitfähigkeit des Tonsubstrates kaum Sicker- 
wasser. Das Sickerwasser müsste außerdem in den größeren Bodentiefen zeitlich versetzt im Vergleich zu den geringeren Bodentiefen ankommen. Das ist nicht der Fall. Die Betrachtung der Einzelwerte (Anhang A) liefert keine zusätzliche Erkenntnis, da es sich nicht um Tageswerte, sondern um Summenwerte für einige Tage handelt.

Die Niederschlagssumme von Oktober bis einschließlich März (Winterhalbjahr) betrug 327,2 mm. Die winterliche Evaporation für den Boden liegt in der Region Südniedersachsen etwa bei 60 mm (HASE \& MEYER, 1969). Nach AG BODEN (1994) beträgt die nFkWe, 110-145 mm für Tu2 und Ld3. Es ist davon auszugehen, dass der Boden bei der genannten maximalen Ausschöpfung im Winterhalbjahr mit den etwa verbleibenden $270 \mathrm{~mm}$ (= Niederschlag - Evaporation) einen maximalen Grad der Aufsättigung erreicht hatte. Es blieben dann 182,2217,2 mm für die Sickerwasserspende. In diesem Wertebereich lag nur das Lysimeter 69 mit $258 \mathrm{~mm}$ Sickerwasser im Winterhalbjahr. Auch im aufgesättigten Zustand gab es erhebliche Sickerwassermengen. Daraus ist zu schließen, dass der Boden im gesättigten Zustand nicht dicht quillt und deshalb der Wassertransport nicht mit einem Ein-Regionen-Modell zu beschreiben ist. Präferenzielle Flüsse sind auch im gequollenem Zustand weiterhin dominierend. Dieser Befund wird von BOUMA \& BOOLTINK (1990) bestätigt, sie fanden bei bodenphysikalischen und morphologischen Untersuchungen an niederländischen Tonböden mit ähnlich hohen Tongehalten, dass sich die wasserführenden Makroporen unter Sättigung nicht völlig schließen und auch im gequollenen Zustand ein präferenzieller Fluss möglich ist.

In Tabelle 6 ist eine Bewässerungsphase dargestellt. Bei allen Lysimetern blieb die Sickerwasser-Wiederfindungsrate deutlich hinter dem Wert des Niederschlages und der Bewässerung zurück. Die Unterschiede in der Wiederfindungsrate der einzelnen Lysimeter untereinander waren sehr groß. Wenn man die tägliche Evaporation auf maximal $2 \mathrm{~mm}$ ansetzt, so ist das Lysimeter S 60 mit Sickerwassermengen von 3,4-4,7 der erwarteten Sickerwassermenge am nächsten. Andere Sickerwassermengen, wie die der Lysimeter S 30 und S 40, blieben weit hinter den zu erwartenden Sickerwassermengen zurück. 
Tabelle 6: Tageswerte von Niederschlag, Beregnung und Sickerwasser Anfang März 1997

\begin{tabular}{rrrrrrrrr}
\hline Datum & N + B & S 30 & S 40 & S 50 & S 60 & S 70 & S 80 & S 90 \\
\hline 05.03. & 5 & 0,0 & 0,0 & 0,0 & 1,5 & 1,1 & 0,4 & 1,2 \\
06.03. & 5,1 & 1,0 & 0,5 & 0,7 & 4,0 & 0,6 & 3,0 & 2,4 \\
07.03. & 5 & 1,3 & 1,3 & 1,3 & 4,7 & 3,4 & 4,3 & 2,8 \\
08.03. & 5 & 0,7 & 0,8 & 0,6 & 3,4 & 2,5 & 2,4 & 2,6 \\
09.03. & 5,1 & 0,9 & 2,0 & 1,9 & 4,5 & 3,2 & 3,1 & 3,4 \\
10.03. & 5 & 0,8 & 0,8 & 0,7 & 3,9 & 2,7 & 2,7 & 2,9 \\
11.03. & 5 & 1,0 & 0,9 & 0,7 & 3,5 & 2,8 & 3,4 & 2,5 \\
12.03. & 5 & 1,4 & 1,5 & 1,4 & 3,8 & 3,4 & 4,0 & 3,5 \\
13.03. & 5 & 1,1 & 0,4 & 1,1 & 4,1 & 3,6 & 4,2 & 3,6 \\
14.03. & 0 & 0,4 & 0,9 & 0,5 & 3,5 & 2,7 & 3,3 & 2,3 \\
\hline Summe & 45,2 & 8,4 & 9,1 & 8,8 & 37,0 & 26,1 & 30,8 & 27,0 \\
\hline
\end{tabular}

In der ersten Versuchsphase mit natürlichen Niederschlägen wurde die Höhe der einzelnen Wiederfindungsraten sehr von der Intensität der einzelnen Niederschlagsereignisse beeinflusst. Um die Variation der Niederschlagsintensität zu eliminieren, wurde die Ackerfläche $\left(30 \mathrm{~m}^{2}\right)$ mit einer Rate von täglich $5 \mathrm{~mm}$ mittels Gießkanne bewässert. Um die natürlich fallenden Niederschläge von der Anlage fernzuhalten und die Verdunstung zu minimieren, wurde die gesamte Ackerfläche mit einer Plane abgedeckt. In Abbildung 4 sind die Sickerwasserraten dieser Bewässerungsphase gegen die Tage (vom 14.-24 April) aufgetragen. Das Sickerwasser vom 14.4. ist der vorhergehenden Beregnung vom 9. und 10. April zuzuordnen. Das wird besonders aus der deutlichen Abnahme von S 70, S 80 und $S 90$ zum 15.4. deutlich. Etwa ab dem vierten Tag zeigen alle Lysimeter den Endwert, d.h., dass sich nach vier Tagen bei der Beregnungssumme von $20 \mathrm{~mm}$ ein neues Fließgleichgewicht eingestellt hat. Nur das Lysimeter S 60 erreichte eine akzeptable Wiederfindungsrate von $90 \%$. Alle anderen Lysimeter hatten niedrigere Wiederfindungsraten, die niedrigste hatte das Lysimeter S 50 mit $34 \%$. Für Wasserhaushaltsuntersuchungen sind diese Werte nicht akzeptabel. 


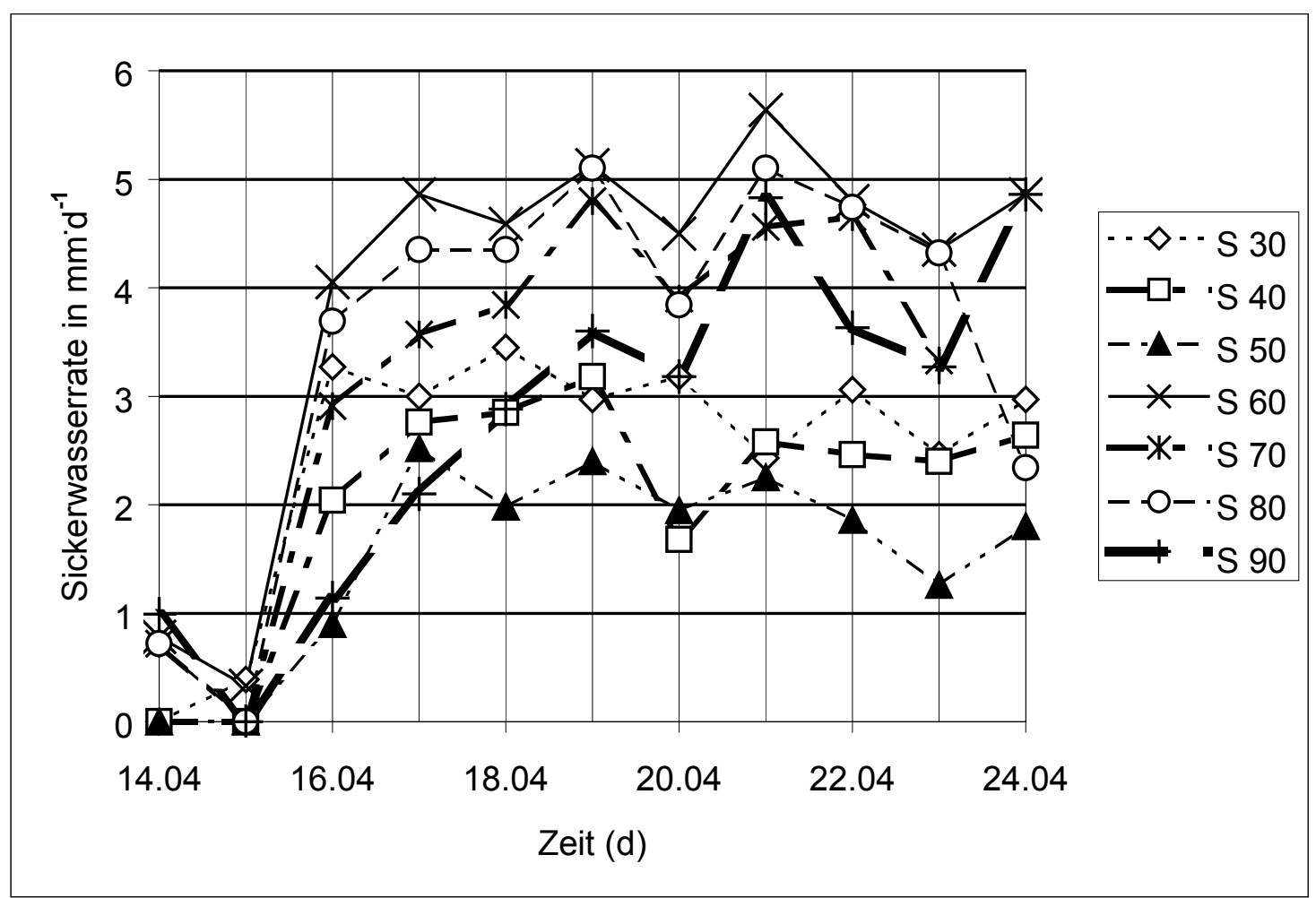

Abbildung 4: Tägliche Sickerwasserraten in den Plattenlysimetern in $30-90 \mathrm{~cm}$ Bodentiefe bei einer Beregnungsrate von $5 \mathrm{~mm}^{-1}{ }^{-1}$, Verdunstung ist ausgeschlossen

Zwischen dem 5. Mai und dem 13. Mai gab es ein Starkregenereignis mit 41 $\mathrm{mm}$. Obwohl die gesamte Anlage mit einer Plane abgedeckt war, wurde im 70 cm tiefen Lysimeter 32,2 mm Sickerwasser gemessen. Die Lysimeteranlage lag an einem Hang mit etwa 3 \% Hangneigung nach Süden (Abbildung 3). Das Wasser, welches das $70 \mathrm{~cm}$ tiefe Lysimeter erreichte, musste mehr als 3 Meter den Hang abwärts in den Boden infiltriert sein, da die umliegende Fläche mit einer Plane abgedeckt war. Das ist ein erster Beweis dafür, dass starke laterale Flüsse vorhanden sind.

Es folgen einige Betrachtungen zum wasserführenden Porenraum in Pelosolen. Für die hydrologischen Parameter sind die Gesamtporenvolumina und die Porengrößenverteilung wichtig. In der Bodenmechanik ist es üblich, die Veränderungen von Aggregaten nicht mit der Veränderung des Porenvolumens zu beschreiben, sondern mit der Porenziffer (HARGTE \& HORN, 1991). Dabei wird das Porenvolumen nicht auf das Gesamtvolumen bezogen, sondern auf das Volumen der Festsubstanz. Die Veränderung von Aggregaten ist entscheidend, da solche Aggregate, wenn sie erst einmal verdichtet sind, sich nur äußerst lang- 
sam regenerieren. Dabei ist der wasserführende Porenraum entscheidend. Im Pelosol ist dies der Interaggregatraum, wenn man postuliert, dass der IntraAggregatraum wegen der geringen Äquivalentporendurchmesser nur eine geringe Beteiligung am Gesamtwasserfluss hat. Dieser Interaggregatraum wurde im Versuch durch das Umgraben im Herbst in den oberen $28-30 \mathrm{~cm}$ wesentlich verändert (Tabelle 7). Außerdem können die Bodenaggregate durch die Pedogenese verändert werden.

Tabelle 7: $\quad$ Tiefenverteilung der Trockendichte, Wassergehalte, Gesamtporenvolumen und der Wasserziffer aus einer im Herbst umgegrabenen Fläche im Sommer 1997

\begin{tabular}{lccccc}
\hline $\begin{array}{l}\text { Tiefenabschnitt } \\
(\mathrm{cm})\end{array}$ & $\begin{array}{c}\mathrm{TD} \\
\mathrm{g} \cdot \mathrm{cm}^{-3}\end{array}$ & $\begin{array}{c}\mathrm{H}_{2} \mathrm{O} \\
\% \text { Gew. }\end{array}$ & $\begin{array}{c}\mathrm{H}_{2} \mathrm{O} \\
\% \text { Vol. }\end{array}$ & $\begin{array}{c}\mathrm{GPV} \\
\% \text { Vol. }\end{array}$ & $\begin{array}{c}\text { Wasserziffer } \\
(\%)\end{array}$ \\
\hline $0-18$ & & & & & \\
$18-26$ & 0,58 & 22,3 & 16,5 & 78,3 & 76,0 \\
$26-36$ & 1,28 & 19,6 & 31,1 & 51,8 & 64,4 \\
$36-48$ & 1,49 & 20,3 & 37,8 & 43,9 & 67,4 \\
$48-58$ & 1,64 & 18,4 & 37,1 & 38,1 & 59,9 \\
$58-67$ & 1,83 & 18,4 & 41,3 & 30,8 & 59,7 \\
$67-77$ & 1,47 & 19,5 & 35,8 & 44,4 & 64,2 \\
& 1,57 & 19,5 & 38,0 & 40,6 & 64,0 \\
\hline
\end{tabular}

Vergleicht man in Tabelle 7 die obersten beiden Schichten, so kann festgehalten werden, dass die Wasserziffer nach unten hin kaum ab- oder zunimmt, während der volumetrische Wassergehalt zunimmt. Nach eigenen Beobachtungen ist die Ursache darin zu sehen, dass die oberste Bodenschicht durch das Umgraben im Herbst und dem nachfolgenden Winter in viele kleine Bröckelaggregate von einigen Millimeter Durchmesser zerlegt worden ist. Dadurch ist einerseits das Gesamtporenvolumen erhöht worden, der volumetrische Wassergehalt ist jedoch niedriger als in der Probentnahmeschicht darunter.

Andererseits ist die Wasserziffer höher als in allen Schichten darunter, also können die kleinen Aggregate je Einheit Festsubstanz mehr Wasser speichern. Dafür gibt es zwei Erklärungsansätze: Die höhere Wasseraufnahme ist eine Folge der Bodenbearbeitung. Durch die Bearbeitung sind kleinere Aggregate 
entstanden. Kleinere Aggregate haben eine größere spezifische Oberfläche als größere. Wenn wir davon ausgehen, dass die Wasserbewegung und damit auch die Wasseraufnahme in den Aggregaten durch die Textur bedingt gering ist, so könnte unter unseren Klimabedingungen die Wasseraufnahmerate begrenzend auf die insgesamt im Winterhalbjahr aufgenommene Wassermenge sein. Die vergrößerte spezifische Oberfläche der kleinen Bodenaggregate könnte so zu einer höheren Wasseraufnahme führen. In der Schicht $18-26 \mathrm{~cm}$ nimmt die Wasserziffer bereits stark ab. Dieser Bereich ist jedoch auch durch die Bodenbearbeitung erfasst worden, jedoch sind die Polyederaggregate wesentlich größer als in der Schicht 0-18 cm.

Ein anderer und hier zutreffender Erklärungsansatz ist, was die Frostverwitterung direkt an der Oberfläche bewirkt hat, dass dort die Aggregate nicht nur zerkleinert wurden, sondern auch in den Aggregaten durch Eislamellen zusätzliche Mittelporen entstanden sind, die das zusätzliche Wasser je Einheit Festsubstanz speichern (FLÖRKEMEIER, 1990). In Tabelle 7 ist kein Zusammenhang zur Grenze des lateralen Flusses zu sehen. Man hätte erwarten müssen, dass in 0-40 cm Bodentiefe eine sehr gering wasserleitende Schicht ist, die mit der Wasserziffer anschaulich gemacht werden kann. Das ist hier nicht der Fall.

\subsubsection{Schlussfolgerungen zur Anwendung von Plattenlysimetern für Wasserhaushaltsmessungen}

Pelosole aus Röt sind auch im gequollenem Zustand fähig, Wasser relativ schnell zu leiten. Die durch Schrumpfung entstandenen Makroporen schließen nicht vollständig. Damit bleibt der Boden auch im gequollenem Zustand ein mehrregionales Fließmedium. Beim Rückquellen des Bodens kommt es zur Aggregatverdichtung. Für das Rückhaltevermögen gelöster Stoffe bedeutet es, dass die Aggregate durch die geringe Korngröße und durch die zusätzliche Verdichtung nur in sehr geringem Umfang in der Lage sind, gelöste Stoffe aufzunehmen. Das Verhalten des Bodens gegenüber gelösten Stoffen soll im nächsten Arbeitsabschnitt untersucht werden.

Die Wasserbilanzen der Lysimeter sind unstimmig. Die Wasserhaushaltsfunktionen sind deshalb mit der Methode der Plattenlysimeter nicht zu bestimmen.

Es gibt laterale Flüsse, die nicht hangparallel verlaufen. Die Anwendung von Plattenlysimetern für die Messung der Sickerwasserbildung ist nicht zulässig. 
Die Einzugsgebiete der einzelnen Lysimeter sind unklar. Aus diesem Grunde ist die Möglichkeit, die Lysimeter für die Messung der Stoffverlagerung zu nutzen, sehr eingeschränkt. Dafür müssen die Einzugsgebiete der einzelnen Lysimeter lokalisiert werden. Hierzu sollen im folgenden Tracerversuche durchgeführt werden.

\subsection{Untersuchungen zum Stofftransport}

Die Tiefenstaffelung der Plattenlysimeter wurde gewählt, um die Infiltrationsraten einzelne Tiefenabschnitte und die Sickerwasserbildung zu messen. Es wurde darüber hinaus erwartet, die Verdrängung der Stoffkonzentration des Sickerwassers zu erfassen und dann mit der Wassermenge die Austragsmenge und Rate je Flächeneinheit zu berechnen. Die Einzugsgebiete der Lysimeter waren, wie bereits dargestellt, unklar, d.h. die Aufstellung einer flächenbezogene Wasserbilanz war nicht möglich. So sollte versucht werden, mit Tracerversuchen die Größe der Lysimetereinzugsgebiete abzuschätzen und festzustellen, wie weit die lateralen Flüsse aus den gedachten Lysimetermonolithen heraus und auf diese hingerichtet reichen.

\subsubsection{Methodik}

\subsubsection{Wahl des Tracers}

Für die Wahl des anzuwendenden Tracers sind folgende Kriterien zu berücksichtigen: Der Tracer soll leicht zu analysieren und umweltverträglich sein. Da es hier nur um die Transportkenngrößen geht, soll der Stoff nicht abgebaut und möglichst nicht sorbiert werden, da es das Ziel ist, den Transport gelöster Stoffe, insbesondere Nitrat, zu simulieren. Ein Farbstoff ist im Sickerwasser gut zu erkennen und kolorimetrisch einfach zu quantifizieren. Viele Farbstoffe werden aber sorbiert (MCLAUGHLIN, 1982). Falls eine Trübung durch Bodenpartikel im Sickerwasser auftritt, kann die kolorimetrische Bestimmung verfälscht werden. BEESE \& VAN DER PLOEg (1979) und BÖTTCHER et al. (1983) haben die Verlagerung von Chlorid und Nitrat miteinander verglichen. SAFFIGNA et al. (1977) haben Chlorid und Bromid als Tracer für Nitrat verglichen. Die genannten Autoren kommen zu dem Schluss, dass Chlorid und Bromid gute Tracer für die Simulation des Nitrattransportes sind. 
Die Wahl fiel deshalb auf Bromid und Chlorid als Tracer. Beim Chlorid muss in der Natur die vorhandene Grundbelastung berücksichtigt werden. Auf landwirtschaftlich genutzten Flächen gibt es hohe Chloridkonzentrationen durch chloridhaltige Düngemittel, wie Kaliumchlorid. Die Versuchsfläche ist seit 1991 nicht mehr in landwirtschaftlicher Nutzung, deshalb dürfte die Chloridgrundbelastung nur dem Niveau der atmospärischen Einträge liegen.

\subsubsection{Durchführung der Tracerversuche}

Die Tracerversuche wurden im Frühjahr 1997 parallel zu den Wasserhaushaltsuntersuchungen (vorheriger Abschnitt) durchgeführt. Es gab zwei Tracerversuche: eine Tracerphase mit Bromid und eine mit Chlorid.

Die Bestimmung des Bromid bzw. Chlorid erfolgte durch potentiometrische Titration mit $\mathrm{AgNO}_{3}$ mit einem Titroprozessor der Firma Methrom.

\section{BROMID-TRACERVERSUCH:}

Beim ersten Tracerversuch wurde das Puls-Verfahren gewählt, damit zu einem späteren Zeitpunkt ein weiterer Tracerversuch durchgeführt werden konnte. Die Messung der Konzentration im Sickerwasser sollte Durchbruchskurven liefern. Dabei wird ein Puls mit $2000 \mathrm{mg}^{-1}$ Bromid mit $1 \mathrm{~mm}$ Wasser ausgebracht. Zur Verlagerung des Tracers wird anschließend mit einer Summe von $60 \mathrm{~mm}$ bei täglich $5 \mathrm{~mm}$ mit destilliertem Wasser beregnet. Mit den natürlich fallenden Niederschlägen ergibt dies eine Summe von $88,9 \mathrm{~mm}$. Das Bromid wurde ausschließlich direkt über den Flächen der einzelnen Plattenlysimeter ausgebracht, um aus dem Bromid-Austrag den Anteil an vertikal fließenden Wasser zu erhalten. Die Bromidfracht, die in den Lysimetern gemessen wird, ist relativ bezogen auf die ausgebrachte Bromidmenge die Wiederfindungsrate. Sie wird in Prozent angegeben und wird zur Bewertung der Güte der Tracerversuche herangezogen. Ideal ist eine Wiederfindungsrate von $100 \%$.

\section{CHLORID-TRACERVERSUCH:}

Zu einem späteren Zeitpunkt wird neben den Lysimetern ein zweiter Tracerversuch angelegt, der speziell die lateral fließende Wassermenge und die Einzugsgebiete neben den Lysimetern sichtbar machen soll. Dieser Versuch wird als Dauertracerversuch angelegt, d.h., dass die Tracerlösung dauerhaft bis zu einer Summe von $70 \mathrm{~mm}$ ausgebracht wird. Zur Markierung lateraler Flüsse wird ein 
1 Meter breiter Streifen $1 \mathrm{~m}$ den Hang abwärts vom Lysimeterkeller mit $1000 \mathrm{mg}^{-1} \mathrm{~L}^{-1}$ Chlorid beregnet (Abbildung 14, S. 76). Der Streifen liegt in $75 \mathrm{~cm}$ Entfernung zum Rand der Plattenlysimeter. Weiterhin wird die restliche im Herbst des Vorjahres umgegrabene Fläche $\left(30 \mathrm{~m}^{2}\right)$ mit destilliertem Wasser beregnet.

Zur Erfassung der Tracerverlagerung wurden auf einer Fläche von $20 \mathrm{~m}^{2}$ in einem Raster von 50 x $50 \mathrm{~cm}$ Bohrungen mit einem Bohrstock mit $10 \mathrm{~cm}$ Durchmesser durchgeführt. Das Bodenmaterial von je $10 \mathrm{~cm}$ Bodentiefe wurde zu einer Probe vereint, die maximale Beprobungstiefe betrug 2 m. $200 \mathrm{~g}$ der feldfeuchten Bodenproben werden bei $105^{\circ} \mathrm{C}$ getrocknet, um den Wassergehalt zu bestimmen. Anschließend wird der getrocknete Boden mit $250 \mathrm{ml} \mathrm{0,01 \textrm {m }}$ $\mathrm{MgSO}_{4} 8$ Stunden lang geschüttelt. Aus der so gewonnenen Lösung wurde das Chlorid bestimmt. Die Wiederfindungsrate wurde folgendermaßen errechnet: Die Konzentrationen der Bodenlösung werden mit den Wassergehalten aus Tabelle 7 verrechnet und auf einen Quadratmeter hochgerechnet.

Es wird ein Verdrängungsmodell angewendet (BÖTTCHER et al. 1983; BACH 1987; GERIES 1991), welches davon ausgeht, dass das Bodenwasser durch infiltrierende Tracerlösung sukzessiv von oben her durch die Tracerlösung nach unten verdrängt wird. Die Wassermenge, die notwendig ist, um das Bodenwasser aus einem gegebenen Tiefenabschnitt zu verdrängen, ist die Feldkapazität. Die Verlagerungsstrecke wird nach dem Konzept der Feldkapazität errechnet.

\subsubsection{Ergebnisse und Diskussion zum Soluttransport}

\subsubsection{Bromid-Tracer-Versuch}

Tabelle 8: $\quad$ Wiederfindungsraten von einem Bromid-Tracer-Puls mit 2000 mg $\mathrm{Br}^{-} \mathrm{L}^{-1}$ nach $187 \mathrm{~mm}$ Niederschlag und Beregnung.

\begin{tabular}{lrrrrrrr}
\hline $\begin{array}{l}\text { Wiederfindungs- } \\
\text { raten }\end{array}$ & Lys 30 & Lys 40 & Lys 50 & Lys 60 & Lys 70 & Lys 80 & Lys 90 \\
\hline$\%$ & 7,7 & 0,2 & 3,5 & 8,1 & 2,6 & 0,9 & 3,3
\end{tabular}

Die Wiederfindungsraten des Bromid-Tracer-Versuchs in den einzelnen Lysimetern lagen zwischen 0,2 und 8,1 \% (Tabelle 8). Der Versuch zeigt, dass unter natürlichen Niederschlagsbedingungen das Wasser überwiegend lateral 
fließt. Solche lateralen Flüsse sind in Tonböden schon beobachtet worden (GAESE, 1979), andererseits ist besonders hervorzuheben, dass dieser laterale Fluss auch im feldgesättigten Zustand auftritt. Vor dem Versuch wurde erwartet, dass laterale Flüsse nur im sommertrockenen Zustand in Schrumpfrissen stattfinden, die mit zunehmender Bodentiefe eine geringeren Äquivalentporendurchmesser haben. Sinnvolle Durchbruchskurven lassen sich mit so niedrigen Wiederfindungsraten nicht erstellen.

\subsubsection{Chlorid-Tracer-Versuch}

Abbildung 5 zeigt zwei repräsentative Chloridtiefenprofile aus Bohrungen, die direkt an den Lysimetern, also $75 \mathrm{~cm}$ neben dem Tracerstreifen genommen wurden. Es gibt Bereiche, in denen sehr niedrige Chlorid-Konzentrationen anzutreffen sind. Die Chlorid-Grundbelastung des Sickerwassers liegt bei etwa $5 \mathrm{mg}^{-1}$. Dennoch gibt es Bodenproben, die unter $1 \mathrm{mg}^{-1}$ Chlorid enthalten wie die Tiefen 140-200 neben dem $90 \mathrm{~cm}$ tiefen Lysimeter in Abbildung 5. Dies ist auf die Bewässerung mit destilliertem Wasser zurückzuführen, oder auf den Sachverhalt, dass dort das Niederschlagswasser wie auch das Tracerwasser nicht hingelangt. Auf einen Abzug der Grundbelastung wurde deshalb verzichtet. Die Unklarheiten bei den Wasserbilanzen aus Tabelle 5 können folgendermaßen erklärt werden: Am $90 \mathrm{~cm}$ tiefen Lysimeter ist in der Schicht 90-100 cm ein deutlicher Chlorid-Konzentrationsanstieg zu verzeichnen. Dieses Ansteigen kennzeichnet eine laterale Fließbahn aus dem Tracerfeld. Es wird davon ausgegangen, dass eine solche Fließbahn für die Wasserbilanzüberschüsse verantwortlich ist.

Am $40 \mathrm{~cm}$ tiefen Lysimeter ist der laterale Fluss in der Bodentiefe von 50-60 cm zu finden. Zum Lysimeter hin ist kein lateraler Zufluss aus dem Tracerfeld erkennbar. Das Einzugsgebiet der tieferen Lysimeter ist größer als für die in näher an der Bodenoberfläche gelegenen Lysimeter. Über dem $40 \mathrm{~cm}$ tiefen Lysimeter muss also auch eine solche Solifluktionslage vorhanden sein. Beim Abbohren des Beregnungsfeldes fielen bereits die mechanisch dichteren Solifluktionslagen auf. Die Solifluktionslagen waren z.T. mechanisch so dicht, so dass man sogar mit einem speziellen Bohrkopf für steinige Böden nicht in der Lage war, bis auf $2 \mathrm{~m}$ zu bohren. Man kann verfolgen, wie das Wasser lateral auf den 
Solifluktionslagen fließt. Dies geschieht unabhängig von der Hangneigung, die entgegengesetzt verläuft.
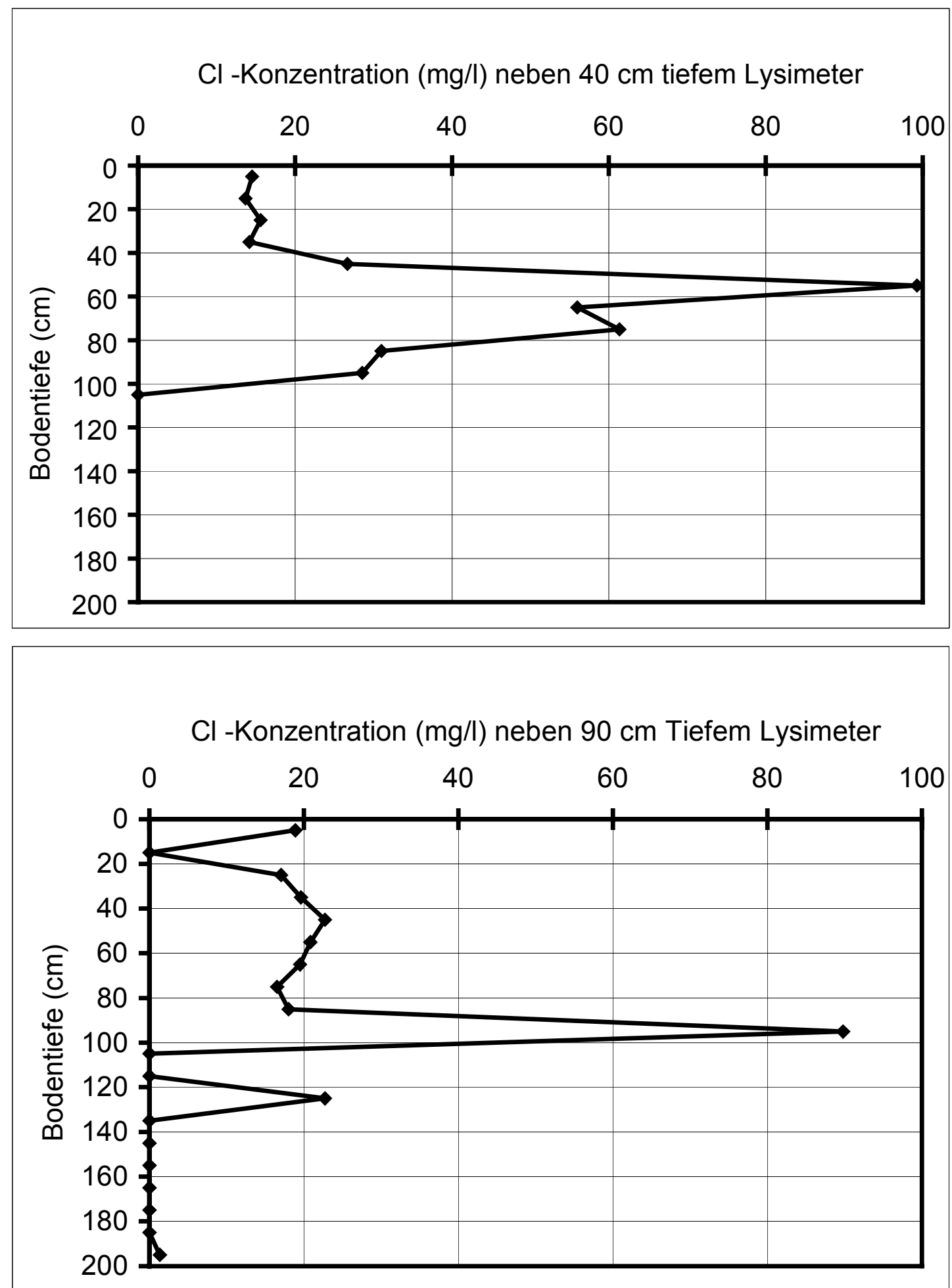

Abbildung 5: Tiefenverteilung der Chloridkonzentrationen in der Bodenlösung neben dem $40 \mathrm{~cm}$ und dem $90 \mathrm{~cm}$ tiefen Plattenlysimeter; Lage: $75 \mathrm{~cm}$ neben dem Tracerstreifen 
Die vertikale Verlagerung des Tracers ist in Abbildung 6 aufgetragen. Es sind vier Chloridprofile unter dem Tracerstreifen neben dem $60 \mathrm{~cm}$ tiefen Lysimeter aufgetragen.

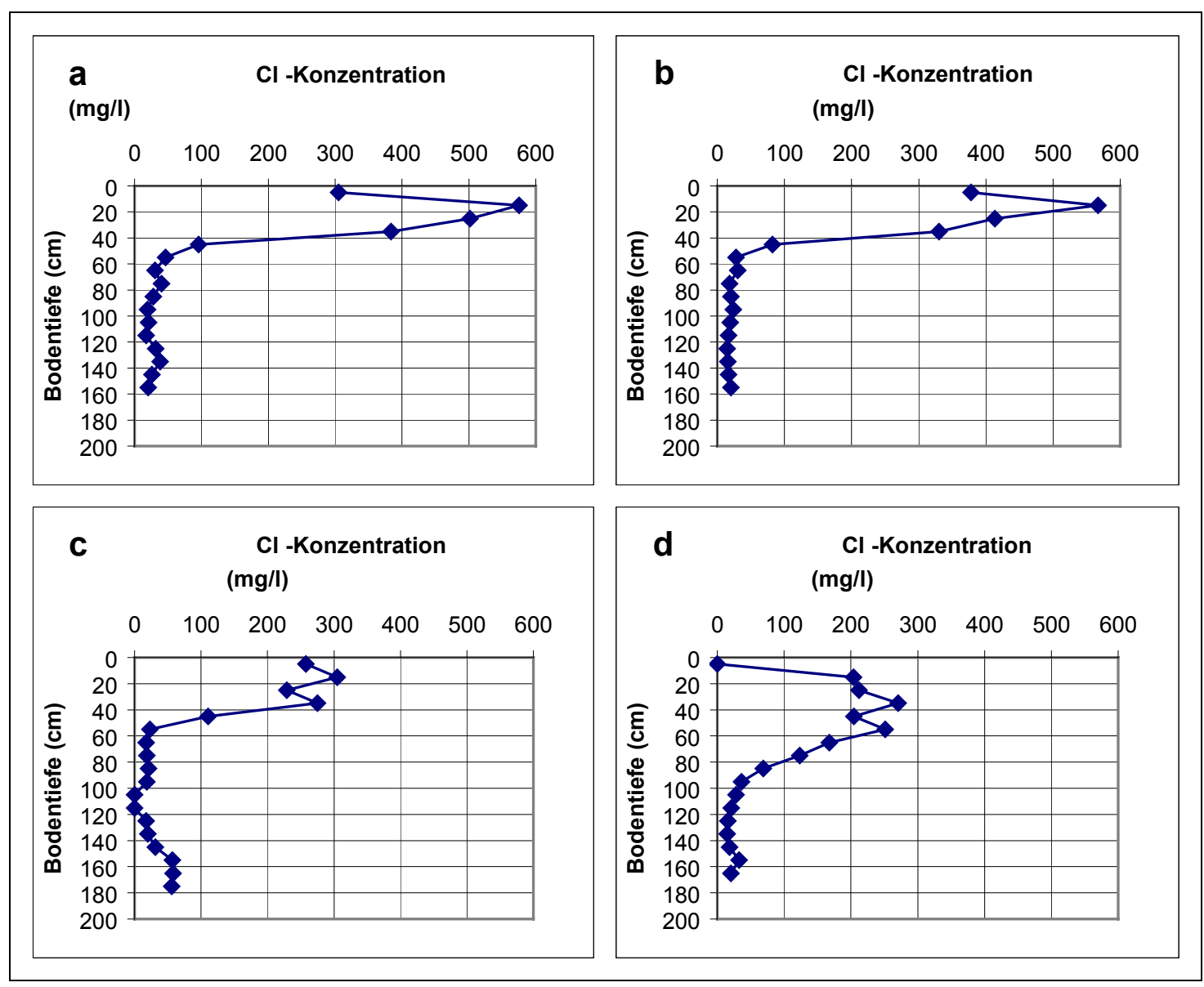

Abbildung 6: Chloridkonzentrationen in der Bodenlösung in Abhängigkeit von der Bodentiefe neben dem $60 \mathrm{~cm}$ tiefen Plattenlysimeter unter den Tracerstreifen (70 mm mit 1000 mg Chlorid je Liter

Die Chloridkonzentrationen in der Ackerkrume und im direkt anschließenden Bereich sind deutlich erhöht im Bereich von ca. 200-600 mg $\mathrm{L}^{-1}$ gegenüber einer Ausgangskonzentration von $1000 \mathrm{mg} \mathrm{L}^{-1}$ Chlorid. Bei allen Profilen ist die Konzentration an der Bodenoberfläche geringer als darunter. Das kann nur durch die Versuchsanordnung erklärt werden: Der Bereich der obersten Bodenschicht erwärmte sich unter der Plane stark. Die Bodenluft und die Luft unter der Plane kann nun aufgrund der höheren Temperatur mehr Wasser als im kalten Zustand aufnehmen. Die Folge ist, dass bei jeder Bewässerung die oberste Bodenschicht stark ausgetrocknet ist. Dadurch ist der Boden geschrumpft, es entstehen zusätzliche Makroporen, und der Wasserfluss kann diese Schicht entspre- 
chend schnell passieren. Weiterhin gibt es bei ausgetrockneten Böden gegenüber vorbefeuchteten Böden einen höheren Benetzungswiderstand, der eine zeitliche Verzögerung der Wasseraufnahme bewirkt. Dieser Benetzungswiderstand, der vor allem in sandigen Böden bekannt ist (DEKKER \& RITSEMA, 1994), wurde auch für Tonböden beschrieben (DEKKER \& RITSEMA, 1996).

In den darunter befindlichen Schichten fließt das Wasser langsamer, die Verweildauer in der Schicht ist länger und somit kann mehr Chlorid in die Bodenaggregate diffundieren und aufgenommen werden. Da der Boden darunter nicht ausgetrocknet ist, kann er auch schneller chloridhaltiges Wasser aufnehmen. Das Ergebnis ist dann eine höhere Konzentration in der Bodenlösung.

Bei den Profilen a, b und $\mathrm{c}$ ist eine rapide Abnahme der Chloridkonzentration im Bereich von 40-50 cm Bodentiefe, bei Profil d eine allmähliche Abnahme im Bereich von 60-100 cm Bodentiefe zu beobachten. Das zeigt zwei wichtige Sachverhalte: Erstens kann daran die Heterogenität der Fließmuster gesehen werden. Diese vier Bohrpunkte liegen alle auf einem Quadratmeter. Sie zeigen sowohl im Ober-, als auch im Unterboden starke Konzentrationsunterschiede. Die Bohrung d hat bis in $1 \mathrm{~m}$ stark erhöhte Chlorid-Konzentrationen, die anderen Bohrungen nur bis in ca. $50 \mathrm{~cm}$. Unter der ehemaligen Ackerkrume, also unter $20 \mathrm{~cm}$ befindet sich ungestörter P-Horizont. Dieser Bereich zeichnet sich durch Prismen und Polyeder-Aggregate (siehe Kapitel 3) mit 5-15 cm Durchmesser aus. Die Interaggregaträume schließen sich beim Quellen des Bodens nicht vollständig. Eine solche weite, kluftähnliche Pore oder ein Regenwurmgang ist anscheinend bei Bohrung d getroffen worden. Es wird davon ausgegangen, dass der Durchmesser des Bohrers mit $10 \mathrm{~cm}$ nicht immer eine solche Wasserleitungsbahn vollständig trifft. Zu den hohen Konzentrationen im Profil d kommt noch hinzu, dass auch in den anderen Profilen die Konzentrationen bis in $2 \mathrm{~m}$ Bodentiefe erhöht sind.

Es ist aus den Profilen ersichtlich, dass im Bereich der Ackerkrume eine hohe Chloridakkumulation stattfindet. Die Wiederfindungsrate im Bereich 0-40 cm beträgt 49,9 \% im Durchschnitt aller Bohrungen. Die Beispiele zeigen aber auch, dass Untersuchungen zur Stoffverlagerung im Feldmaßstab in Pelosolen wegen der bevorzugten Abflussbahnen besonderen Schwierigkeiten unterliegen, da die wasserführenden kluftartigen Poren oder Regenwurmgängen eine 
Probenentnahmegröße von mehreren Dezimetern erfordern. Zusätzlich wird die Aufklärung der Transportpfade für gelöste Stoffe durch die gezeigten lateralen Flüsse erschwert.

Tabelle 9: $\quad$ Tiefenverteilung der Wassergehalte und der daraus errechneten Bodenwassermenge in $\mathrm{mm}$ auf einer im Herbst 1996 umgegrabenen Fläche im Frühjahr 1997; Ackerkrume: 0-30 cm

\begin{tabular}{lcc}
\hline Tiefenabschnitt (cm) & $\begin{array}{c}\mathrm{H}_{2} \mathrm{O} \\
\% \text { Vol. }\end{array}$ & $\begin{array}{c}\mathrm{H}_{2} \mathrm{O} \\
(\mathrm{mm})\end{array}$ \\
\hline $0-18$ & 16,5 & 29,7 \\
$18-26$ & 31,1 & 24,9 \\
$26-36$ & 37,8 & 37,8 \\
$36-48$ & 37,1 & 44,8 \\
$48-58$ & 41,3 & 41,3 \\
$58-67$ & 35,8 & 32,2 \\
$67-77$ & 38,0 & 38,0 \\
\hline
\end{tabular}

In Tabelle 9 sind die Wassergehalte aus dem vorherigen Kapitel aufgetragen. Das gespeicherte Wasservolumen je Tiefenabschnitt ist auf einen Quadratmeter hochgerechnet. In den obersten zwei Schichten (0 bis 18 und 18 bis $26 \mathrm{~cm}$ ) sind demzufolge 54,6 mm Wasser gespeichert. Demgegenüber sind $70 \mathrm{~mm}$ mit Tracerlösung beregnet worden. Bei der Anwendung des Verdrängungsmodells (GERIES, 1991) müssten die oberen $26 \mathrm{~cm}$ komplett und vom darunterliegenden Tiefenabschnitt teilweise mit Tracerlösung ausgetauscht worden sein. Demzufolge muss nach dem Verdrängungsmodell der Boden unter etwa $30 \mathrm{~cm}$ Bodentiefe chloridfrei sein. In Abbildung 7 a, b und c zeigt sich unter $30 \mathrm{~cm}$ Bodentiefe eine Abnahme der Konzentrationen auf weniger als $50 \mathrm{mg} \mathrm{L}^{-1}$, in Abbildung $7 \mathrm{c}$ ist diese Abnahme erst bei $90 \mathrm{~cm}$ zu beobachten. Berücksichtigt man weiterhin, dass die obersten $10 \mathrm{~cm}$ Boden weniger Tracerwasser aufgenommen haben und das infiltrierte Wasser gleich in größere Bodentiefen gelangte, so ergeben die Abbildungen 7 a, b und c ein befriedigendes Ergebnis für das Verdrängungsmodell, wenn auch die maximal erreichten Konzentrationen in der Bodenlösung weit hinter der ausgebrachten Tracerkonzentration zurückbleiben. 
In Abbildung 7 ist ein weiteres, typisches Chloridtiefenprofil im Tracerfeld abgebildet. Hier ist zu erkennen, dass es Tiefenabschnitte gibt, in denen kein chloridhaltiges Wasser in die Matrix gelangt ist. Es ist der Bereich $40-50 \mathrm{~cm}$, in dem keine messbare Chloridkonzentration anzutreffen sind. Darunter sind alle gemessenen Tiefenabschnitte in der Konzentration gegenüber der Grundbelastung erhöht. Die Vermutung liegt nahe, dass einzelne Tiefenabschnitte, vermutlich Solifluktionslagen, nahezu porenfrei sind und andere Bereiche sehr gute Meso- oder Makroporen entwickelt haben.

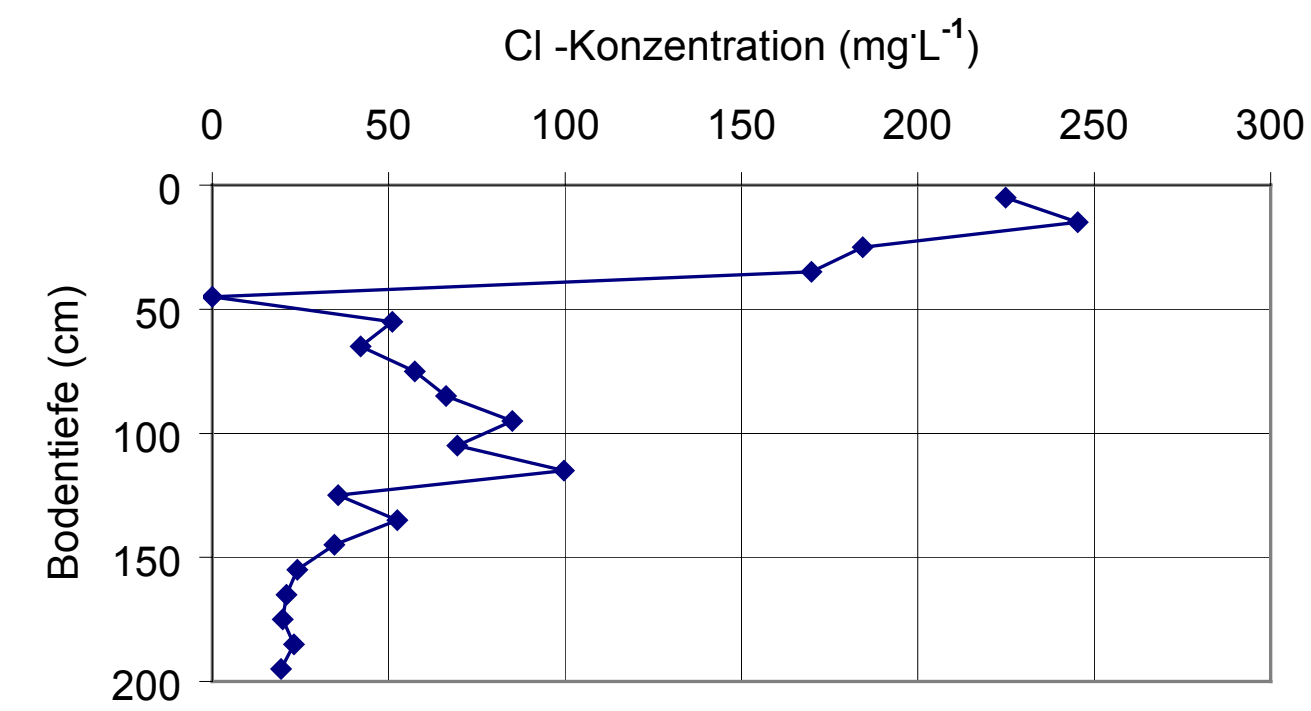

Abbildung 7: Chloridkonzentrationen in der Bodenlösung in Abhängigkeit von der Bodentiefe neben dem $70 \mathrm{~cm}$ tiefen Plattenlysimeter (Bohrung 47) unter einen Tracersteifen nach Beregnung von $70 \mathrm{~mm}$ mit $1000 \mathrm{mgL}^{-1}$ Chlorid

\subsubsection{Betrachtungen zum Partikeltransport}

Ein wichtiges Ziel dieser Arbeit ist, den Stofftransport in Pelosolen vor allem in Hinblick auf die wasserwirtschaftliche Nutzung zu untersuchen. Neben dem Soluttransport ist für die Wasserqualität auch der partikuläre korpuskuläre Stofftransport von Bedeutung. Im Folgenden soll der Begriff Partikeltransport verwendet werden. Für die Trinkwasserqualität ist dabei nicht wie bei den gelösten Stoffen die Höhe der in das Grundwasser eingetragenen Stoffkonzentra- 
tion entscheidend, sondern es interessiert wegen der äußerst niedrigen zulässigen Konzentration im Trinkwasser eher, ob der Stoff eingetragen wird oder nicht. Ursprünglich war an die Aufbringung von Bakterien oder Microspheres gedacht. Diese relativ aufwendigen Verfahren wurden wegen der Unstimmigkeiten bei den Wasserhaushaltsbilanzen zurückgestellt. Dennoch haben sich bei der weiteren Versuchsdurchführung für den Partikeltransport in Pelosolen wichtige Erkenntnisse ergeben, die hier dargestellt werden sollen.

Die Fragestellung, ob ein Partikeltransport stattfinden kann, ist durch das Vorhandensein von Tontapeten bis in $90 \mathrm{~cm}$ Bodentiefe, bestehend aus braunem Ton aus dem Oberboden, eindeutig positiv zu beantworten. Nach der Vorstellung von DIEZ (1959) entstehen solche Tapeten im geschrumpften Zustand dadurch, dass suspendierter Ton in die Schrumpfrisse infiltriert. Anschließend quillt der Boden und es kommt durch die zusätzlich pro Raumeinheit vorhandene Bodenmenge zur Aggregatverdichtung. Der Transport geschieht nach DIEZ (1959) nur im ausgetrockneten und geschrumpften Zustand. Im feldgesättigten Zustand sollen die Makroporen geschlossen sein. Es stellt sich daher die Frage nach dem Transport unter feldgesättigten Bedingungen. Die ChloridTracerversuche (Kapitel 4.2.3) haben im Zustand der Feldsättigung gezeigt, dass ein präferenzieller Transport stattfindet, der auch nach einer Beregnungsmenge von $70 \mathrm{~mm}$ eine Bodentiefe von $200 \mathrm{~cm}$ (Abbildung 7) erreicht. Es ist die Frage zu beantworten, ob unter diesen Bedingungen auch Partikel bis unter die ET-Zone gelangen können.

Die Frage nach dem Transport von Tonpartikeln kann durch den Tonaustrag aus den Plattenlysimetern beantwortet werden. Es handelt sich hierbei um braunen Ton, der aus dem Oberboden stammt und mobilisiert worden sein muss. Durch die Beregnung mit destilliertem Wasser begann der Boden im Frühjahr 1997 oberflächlich zu verschlämmen. Um weiterhin eine vertikale Infiltration zu gewährleisten, wurde die Versuchsfläche mit einem Gartengrubber am 14. März 1997 vorsichtig oberflächlich aufgelockert. In den folgenden sechs Tagen fielen mit künstlicher Beregnung 38,9 mm Niederschlag. In diesem Zeitraum wurde im Sickerwasser plötzlich eine deutliche Trübung durch braunen Oberbodenton beobachtet. Die Korngrößenanalyse nach der Sedimentationsmethode nach Atterberg (SCHLICHTING et al. 1995) ergab folgende Ergebnisse: Die höchste Schwebstoffkonzentration an braunem Ton beträgt $0,71 \mathrm{~g}^{-1}$ in 
$30 \mathrm{~cm}$ Bodentiefe, selbst in $70 \mathrm{~cm}$ sind noch bis zu 0,19 $\mathrm{g} \mathrm{L}^{-1}$ gemessen worden. Die verlagerten Frachten stiegen nach einem großen Niederschlagsereignis am 17. März (Tabelle 10) stark an, am folgenden Tag wurden auch die höchsten Konzentrationen gemessen. Es zeigt sich also, dass die hohe Niederschlagsenergie eines Ereignisses zur Verlagerung von Partikeln aus dem Oberboden ausreicht.

Tabelle 10: $\quad$ Sickerwasserfrachten von braunem Ton $\left(g^{-1} L^{-1}\right)$ in Plattenlysimetern verschiedener Bodentiefen und Niederschläge $(N)$ im Zeitraum vom 15.3.-2.4.1997 in g (leere Felder = nicht nachweisbar)

\begin{tabular}{|c|c|c|c|c|c|c|c|c|c|c|}
\hline $\begin{array}{l}\text { Tiefe der } \\
\text { Lysimeter }\end{array}$ & \multicolumn{3}{|c|}{$30 \mathrm{~cm}$} & $40 \mathrm{~cm}$ & \multicolumn{2}{|c|}{$50 \mathrm{~cm}$} & \multirow{2}{*}{\begin{tabular}{|r|}
$60 \mathrm{~cm}$ \\
$<2,0$
\end{tabular}} & \multirow{2}{*}{\begin{tabular}{|l|}
$70 \mathrm{~cm}$ \\
$<2,0$
\end{tabular}} & \multirow{2}{*}{\begin{tabular}{|r|}
$80 \mathrm{~cm}$ \\
$<2,0$ \\
\end{tabular}} & \multirow{2}{*}{$\begin{array}{c}\mathrm{N} \\
(\mathrm{mm})\end{array}$} \\
\hline $\begin{array}{c}\text { Korn- } \\
\text { größe } \\
(\mu \mathrm{m})\end{array}$ & $>0,63$ & $\begin{array}{l}0,63 \\
-2,0\end{array}$ & $<2,0$ & $>0,63$ & $>0,63$ & $<2,0$ & & & & \\
\hline 15.03 .97 & 0,018 & & 0,053 & & & & & & & 6,2 \\
\hline 16.03 .97 & 0,097 & 0,049 & 0,016 & & & & & & & 8,2 \\
\hline 17.03 .97 & & & & & & & & & & 18,0 \\
\hline 18.03.97 & 0,543 & 0,310 & 0,310 & 0,224 & 0,219 & 0,219 & 0,127 & 0,619 & 1,074 & 0,1 \\
\hline 20.03 .97 & 0,075 & 0,030 & 0,060 & & & & & & & 6,4 \\
\hline 02.04 .97 & & & & & 0,045 & 0,015 & & & & 16,2 \\
\hline $\bar{\Sigma}$ & 0,733 & 0,398 & 0,440 & 0,224 & 0,264 & 0,234 & 0,127 & 0,619 & 1,074 & 55,1 \\
\hline
\end{tabular}

Die durchschnittlichen Schwebstoffkonzentrationen der einzelnen Lysimeter liegen in dieser Zeit zwischen 0,05-0,37 g: $\mathrm{L}^{-1}$. Im Vorfluter eines ähnlichen Gebietes wurden bis zu $38 \mathrm{~g} \mathrm{~L}^{-1}$ während einer Hochwasserwelle nach sommerlichen Starkniederschlagsereignissen gemessen (PÖRTGE, 1979).

Rechnet man die Schwebstoffkonzentrationen des $60 \mathrm{~cm}$ tiefen Lysimeters von der Auffangfläche der Lysimeterplatte hoch auf einen Hektar, so ergeben sich

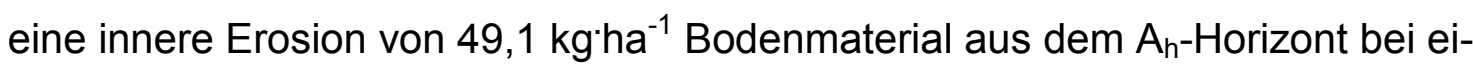
ner Niederschlagssumme von 38,9 mm. Selbst im $80 \mathrm{~cm}$ tiefen Lysimeter werden auf einen Hektar hochgerechnet $33,6 \mathrm{~kg}$ Oberbodenmaterial aufgefangen.

Es stellen sich die Fragen, ob die genannten Austragsmengen realistisch sind und ob diese Zahlen relevant für die Praxis sind. Die Fracht im 60 bzw. $80 \mathrm{~cm}$ tiefen Lysimeter kann als Wert nicht auf einen Hektar hochgerechnet werden, da die Sickerwassermenge, die im betreffenden Zeitraum angefallen ist, $151 \%$ 
über der Niederschlagsmenge lag. Im betreffenden Zeitraum sind in den ersten drei Tagen je $5 \mathrm{~mm}$ an zusätzlicher Beregnung ausgebracht worden. Im Zeitraum vom 1. März bis zum 2. April fielen insgesamt 111,6 mm Niederschlag, gegenüber einem langjährigen Mittel von 39 mm (SCHIRMER \& VENT-SCHMIDT, 1979) für den Monat März. Das bedeutet fast eine Verdreifachung des Monatsniederschlages.

Wenn es jedoch in jedem Jahr zu solchen Ereignissen kommt, können solche Verluste an humosem Oberbodenmaterial für ackerbaulich genutzte Standorte nicht als unwesentlich betrachtet werden.

Die Ergebnisse von Tabelle 10 zeigen außerdem, dass der Partikeltransport von Tonteilchen in Pelosolen auch im feldgesättigten Zustand bis in Bodentiefen von $80 \mathrm{~cm}$ möglich ist. Der weitere Verbleib ist ungewiss, zwei Austragswege sind denkbar: Erstens können die Austräge an Bodenmaterial über Dräne in die Vorfluter gelangen und dort zu einer merklichen Phosphatbelastung führen, da Phosphat an der Festphase des Bodens sorbiert ist. Oberflächengewässer reagieren sehr empfindlich auf die Anhebung der P- Konzentration. Zweitens ist denkbar, dass solche Partikel auch in grössere Bodentiefen gelangen oder durch ausgelaugte Gipslinsen im Röt 1 bis in den Mittleren Buntsandstein gelangen.

Aus der Beobachtung des Tontransportes ist zu folgern, dass ein weitreichender Transport von solchen suspendierten Stoffen ausdrücklich nicht ausgeschlossen werden kann.

\subsubsection{Schlussfolgerungen und Ausblick}

Für die Messung der Stoffflüsse sind Plattenlysimeter nur sehr begrenzt einsetzbar. Der Bilanzierungsansatz kann nicht angewendet werden, weil die Wasserbilanzmengen unstimmig sind. Eine Bestimmung der Einzugsgebiete der einzelnen Lysimeter ist nicht gelungen. Der schlüssige Versuchsansatz war, einen Lysimetertyp zu nutzen, der keine seitlichen Randeffekte hat. Diese Randeffekte sind in Böden mit starker Quellungs- und Schrumpfungsdynamik schwer auszuschließen. Laterale Flüsse sind für den Versuchsstandort typisch und konnten auch im Tracerversuch erkannt werden. Der Tracerversuch lässt auch erkennen, dass das Maximum der Chloridaufnahme in einem 0,25 $\mathrm{m}^{2}$ großem Quadrat stark variiert (Abb. 6). Die Messung von bodenphysikalischen 
Parametern mit gebräuchlichen 100 oder 200 cm³ Stechzylindern kann solche für die Hydrologie in Pelosolen entscheidenden Strukturen nicht erfassen.

Aus den gezeigten präferenziellen Flüssen ist zu folgern, dass ein einfaches Verdrängungsmodell nach dem Konzept der Feldkapazität nicht anwendbar ist. Dies wird von BLAKE et al. (1973) für Pelosole bestätigt.

Zum Aufklären der Fließstrukturen in Pelosolen aus Röt setzte SPANGENBERG (1998) die Computertomographie ein. Sie erlaubte jedoch nur eine Auflösung der Poren bis $300 \mu \mathrm{m}$ in einem Gefäßquerschnitt von $12 \mathrm{~cm}$. Nach unseren bisherigen Erkenntnissen zur Heterogenität ist dieser Querschnitt zu gering, um Aussagen zum Feldmaßstab zu machen. Auch die von DE MoL (1996) angewendete Infiltrometermethode erlaubte nur einen minimalen Porendurchmesser von $300 \mu \mathrm{m}$. Im Folgenden soll versucht werden sowohl dem Maßstab als auch den möglichen vorkommenden Porengrößen gerecht zu werden. Besonders muss das laterale Fließen berücksichtigt werden, was in Pelosolen aus Röt am Versuchsstandort typisch ist.

Hierzu sollen zwei Versuchsansätze dienen. Zum einen sollen die hydrologischen Eingangsparameter in einem eindimensionalen Lysimeterversuch unter Berücksichtigung der lateralen Solifluktionslagen bestimmt werden. Zum anderen sollen auf Feldmaßstabsebene die Solifluktionslagen erkundet und mit begleitenden bodenphysikalischen Messungen hinreichend charakterisiert werden.

Die Versuchsfrage nach dem partikulären- korpuskulären Stofftransport ist für Belange der Landbewirtschaftung folgendermaßen zu beantworten: Die Gefahr, dass feste Stoffe ausgewaschen werden, ist ganzjährig hoch. Andere Autoren stellen die besondere Gefahr der Verlagerung von Stoffen im trockenem Zustand in Schrumpfrissen und anderen Makroporen heraus. Wir können unsere untersuchten Pelosole auch für den feldgesättigten Zustand ein hohes Verlagerungsrisiko festhalten. Schon hier kann festgehalten werden, dass Pelosole aus Röt nicht in einer solchen Weise quellen, dass die durch Schrumpfung entstandenen Makroporen sich schließen. Das ist auch an den präferenziellen Flüssen des Chlorids im feldgesättigten Boden zu sehen (Abbildung 7). Ausgetrocknete Tonböden haben ein hohes Verlagerungsrisiko wegen der Schrumpfrisse mit großen Porendurchmessern und einer hohen Porenkontinuität. Im trockenen 
Zustand kann der Boden jedoch das eindringende Wasser bis zu einem gewissen Grad aufnehmen, wodurch die Auswaschungsgefahr bis zu einer bestimmten Niederschlagsmenge und -rate vermieden wird. Im feldfeuchten $\mathrm{Zu}$ stand ist die Wasseraufnahme für den Boden nicht oder nur sehr begrenzt möglich. Das Wasser gelangt schneller und in größerer Menge durch eine Volumeneinheit Boden. So kann die Oberflächensorption für suspendierte partikuläre Stoffe nicht oder nur sehr schlecht wirksam werden. Auch für Solute gilt, dass erst bei längerer Verweildauer eine Diffusion in die Bodenaggregate und damit ein Zurückhalten der gelösten Stoffe möglich ist. 


\section{Bodenhydrologische Untersuchungen an mo- nolithischen, wassergesättigten Laborlysime- tern}

Die Messung der Transportparameter für den Lösungstransport in Pelosolen steht bei der nachfolgend beschriebenen Versuchsanordnung im Vordergrund. Sie besteht aus monolithischen Lysimetern, synonym werden auch die Begriffe Laborlysimeter oder Bodenmonolithe verwendet.

\subsection{Methodik}

\subsubsection{Methodenwahl}

In Kapitel 4.2 konnte gezeigt werden, wie das Wasser nach der Infiltration zuerst vertikal im Boden fließt und dann auf einer Solifluktionslage lateral zu fließen beginnt. Diese Solifluktionslagen können bereits im Gelände anhand der unterschiedlichen Bodenfarben gut voneinander unterschieden werden.

Pelosole werden in der landwirtschaftlichen Praxis häufig nicht mehr ackerbaulich genutzt, sondern sind stillgelegt. Die im Folgenden beschriebenen Säulenversuche wurden daher unter einer Dauerbrache entnommen, die letztmalig 1991 gepflügt worden ist und seitdem mit Kleegras (Lolium perenne L. und Trifolium repens L.) bewachsen ist. Die Orte, an denen die Lysimeter entnommen worden sind, befinden sich $4 \mathrm{~m}$ südlich von der Plattenlysimeteranlage (siehe Abb. 14).

Es wurden vier monolithische, also ungestörte Lysimeter entnommen, die sich in der Länge unterschieden und dadurch die oberen 30, 31,5, 53,1 und 67,5 cm Boden einschließen. In den kürzeren Lysimetern wurde der ehemalige $A p(P)-$ Horizont etwa 0-20 cm und weitere $10 \mathrm{~cm}$ P-Horizont aufgenommen. In den längeren Lysimetern wurde zusätzlich noch P-Horizontmaterial mit der obersten Solifluktionslage in etwa 35-60 cm Bodentiefe aufgenommen. Im Nachfolgenden wird der Tiefenabschnitt 0-30 cm als P-Horizont, der darunter befindliche Boden als Solifluktionslage bezeichnet.

Randeffekte sind bei Lysimetern mit strukturdynamischen Böden ein besonderes Problem, wie bereits in Kapitel 4.1.2 diskutiert worden ist. Um den Kontakt zwischen Boden und Lysimeterwand für wassergesättigte Versuche herzustellen, wurden die Lysimeter folgendermaßen angefertigt: Als Lysimeterbehälter 
dienten PVC-Rohre mit einem Innendurchmesser von $40 \mathrm{~cm}$. Der Boden wurde abgegraben, so dass zylindrische Monolithe mit einem Durchmesser von $37 \mathrm{~cm}$ stehen blieben. Das PVC-Rohr wurde im Gelände über den Monolithen gestülpt und der Zwischenraum zwischen Boden und PVC-Rohr mit Polyuhrethanschaum ausgeschäumt. Direkt am Bodenkörper befand sich eine Polyethylenfolie, damit der Schaum nicht mit dem Boden in Berührung kam. Der Polyuhrethanschaum übt beim Aushärten einen leichten Druck auf den Bodenkörper aus. Das kann einerseits zu einer leichten Randverdichtung führen, andererseits wird dadurch die Bildung von künstlichen Randporen an der Lysimeterwand und ein gegenüber dem natürlich gelagertem Boden zusätzlicher, präferenzieller Fluss vermieden. Bei einem Durchmesser des Bodenkörpers von $37 \mathrm{~cm}$ wird erwartet, dass der Effekt der Randverdichtung keine große Rolle spielt.

Der Durchmesser von $37 \mathrm{~cm}$ wurde relativ groß gewählt, um die vorhandenen Porenstrukturen und Porengrößenklassen möglichst in allen Gefäßen repräsentativ zu erfassen. Der Heterogenität des Bodens könnte auch bei der Verwendung von gebräuchlichen Stechzylinderproben (100-200 cm³) mit einer größeren Anzahl an Wiederholungen begegnet werden, jedoch können die Strukturen im Bereich der Makroporen in kleineren Proben nicht abgebildet werden. Die gesättigte Leitfähigkeit hängt im Wesentlichen von den Makroporen ab. MESSING \& JARVIS (1995) fanden Unterschiede in der gesättigten Leitfähigkeit zwischen Stechzylinderproben $\left(407,2 \mathrm{~cm}^{3}\right)$ und Bodenmonolithen $\left(39642,6 \mathrm{~cm}^{3}\right)$ in der Größenordnung von zwei Zehnerpotenzen. Unsere Monolithe enthalten je nach Länge zwischen 32256,3 und $74189,5 \mathrm{~cm}^{3}$ Boden. Somit sind sie geeignet, um für Feldbedingungen realistische Kenngrößen abzuleiten.

Die Unterseite der Lysimeter wurde mit einer Bodenplatte verklebt, die Dränung der Monolithe erfolgte durch eine $2 \mathrm{~cm}$ starke Feinkieslage an der Unterseite. In der Bodenplatte befindet sich ein Wasserablauf, damit das ausfließende Wasser aufgefangen werden kann.

\subsubsection{Wasserleitfähigkeit}

Die Messung der gesättigten Wasserleitfähigkeit wird durch Anlegen eines Druckgefälles an das gesamte Lysimeter (Tabelle 11) bestimmt. Das Druckgefälle muss sehr klein gewählt werden, damit keine innere Erosion und damit ab- 
nehmende Wasserdurchflussraten vorkommen, die die hydrologischen Eigenschaften des Bodens verändern. Eine solche innere Erosion trat aufgrund der hohen Permeabilität beim Lysimeter 1 auf (Abbildung 8). Die Wasseraustragsraten nehmen im Versuchsverlauf deutlich ab.

Anschließend wird der Versuch noch einmal mit einem geringerem Druckgefälle durchgeführt, bei dem innere Erosion vermieden wird. Das Druckgefälle in Tabelle 11 ist in der Höhe der Wassersäule angegeben (cm WS).

Die ausfließende Wassermenge wird mit Hilfe einer Waage erfasst und in verschiedenen Zeitintervallen abgelesen.

Tabelle 11: $\quad$ Druckdifferenzen bei der Messung der gesättigten hydraulischen Leitfähigkeit für die verschieden langen Laborlysimeter

\begin{tabular}{lllll}
\hline $\begin{array}{l}\text { Lysimeterlänge } \\
(\mathrm{cm})\end{array}$ & 30 & 30,5 & 51,3 & 69 \\
\hline Druckdifferenz & 0,5 & 2 & 3 & 3,5 \\
$(\mathrm{~cm}$ WS $)$ & & & & \\
\hline *WS $=$ Wassersäule & & &
\end{tabular}

Die Wasserleitfähigkeit (k) wird nach SCHLICHTING \& BLUME (1995) berechnet,

$$
k=\frac{V \cdot l}{F \cdot t \cdot h}\left[\mathrm{~cm} \cdot d^{-1}\right]
$$

wobei V das Wasservolumen $\left(\mathrm{cm}^{3}\right)$, t die Zeit in Tagen, I die Länge des Bodenkörpers, $F$ ist der Fließquerschnitt $\left(\mathrm{cm}^{2}\right)$ und $h$ der hydraulische Gradient $(\mathrm{cm})$ ist. Im wassergesättigten Boden wird die Wasserleitfähigkeit als $k_{s}$ bezeichnet. In nicht strukturierten Böden hängt die wassergesättigte Leitfähigkeit stark von der Lagerungsdichte und vom Gesamtporenvolumen ab. In strukturierten Böden müssen gesättigte Wasserleitfähigkeit und das Gesamtporenvolumen nicht in einer positiven Korrelation zueinander stehen, da die gesättigte Leitfähigkeit maßgeblich von den Grobporen abhängig ist, die u.U. nur einen geringen Volumenanteil ausmachen. Nach AG BODEN (1994) werden Werte der gesättigten Leitfähigkeit von 1-27 $\mathrm{cm}^{\cdot} \mathrm{d}^{-1}$ für die Bodenarten schwach schluffiger Ton (Tu2) und mittel schluffiger Ton (Tu3) erwartet. 


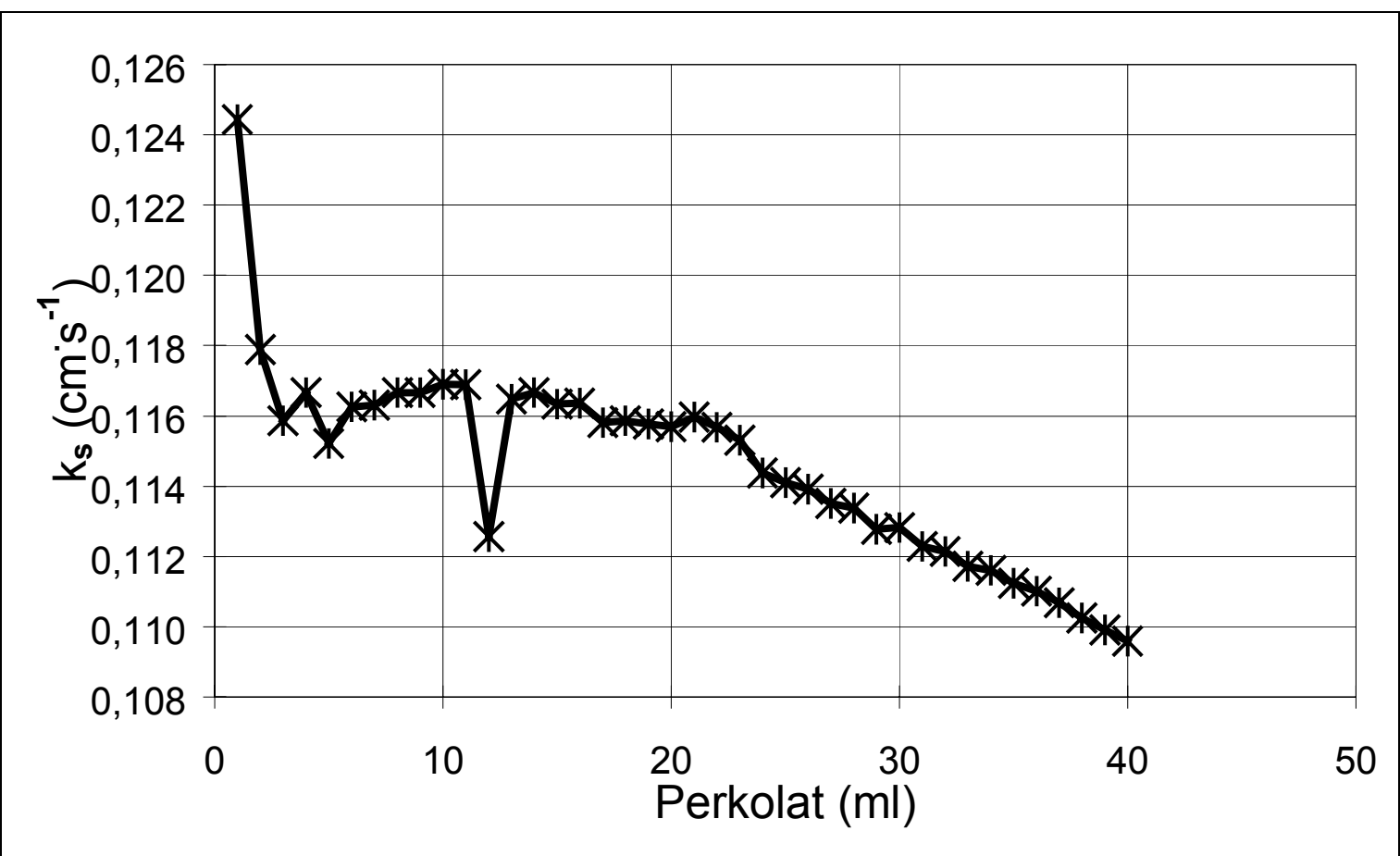

Abbildung 8: Entwicklung der gesättigten Wasserleitfähigkeit bei innerer Erosion bei Lysimeter 30 mit 1,2 cm WS Druckdifferenz

\subsubsection{Chlorid-Dauertracerversuch}

Die Beeinflussung des hydrologischen Verhaltens durch die Matrix und / oder den Sekundärporen soll anhand des Chlorid-Tracerversuches geklärt werden. Der körnungsbedingt geringe Äquivalentporendurchmesser führt dazu, dass die Matrix von schweren Böden im gesättigten Zustand nur einen sehr geringen Beitrag an der Wasserbewegung und am Soluttransport leistet. Im ausgetrockneten Zustand ist die Matrix in der Lage, Wasser und darin gelöste Stoffe direkt aufzunehmen, weil ein Wasserspannungsgradient in Richtung vom freien Wasser zur ausgetrockneten Matrix vorliegt. So kann die Matrix die schnelle Verlagerung in Makroporen mindern. Im feldgesättigten Zustand fehlt dieser Gradient. Ein im Wasser gelöster Stoff kann nur über die Diffusion in das Bodenaggregat gelangen. Die Diffusion in die Matrix ist im Vergleich zur schnellen Wasserbewegung in den Makroporen ein sehr langsamer Prozess und deshalb fast zu vernachlässigen (HOOGHMOED \& BOUMA, 1980).

Die Feldversuche (Kapitel 4) haben gezeigt, dass die Makroporen durch das Quellen des Bodens nicht geschlossen werden. Wenn im trockenen wie im feuchten Zustand die Makroporen als Transportwege zur Verfügung stehen, 
kann angenommen werden, dass die größte Austragsgefahr für Solute im gequollenem Zustand im zeitigen Frühjahr ist. Die Bodenmatrix ist weitgehend von der mobilen Phase ausgeschlossen (BECHER 1985) und kann deshalb Solute nur im geringen Maß zurückhalten. Deshalb wird die Bestimmung des Retentionsverhaltens im gesättigten Zustand durchgeführt. In der Phase der Feldversuche traten einige Niederschlagsereignisse von 10 bis $20 \mathrm{~mm}$ pro Tag auf. Die Perkolationsrate wird auf $15 \mathrm{~mm}$ pro Tag gewählt, also mit der Stärke eines im zeitigen Frühjahr häufig vorkommenden Tagesereignisses. Die Perkolationsrate wird mit einer Schlauchpumpe an den Lysimeterausflüssen eingestellt. Dadurch werden steady-state Bedingungen eingehalten, d.h., dass die Durchflussrate konstant gehalten wird. Der Tracerversuch wird mit den Lysimetern 1, 2 und 3 mit den Längen 30, 53,1 und 69 cm durchgeführt.

Sobald alle Monolithe die Wasserdurchflussrate von $15 \mathrm{~mm}^{\cdot \mathrm{d}^{-1}}$ erreichen, wird auf die Bodenoberfläche der Lysimeter dauerhaft eine Chloridlösung mit der Konzentration von $1000 \mathrm{mg}^{-1} \mathrm{~L}^{-1}$ zugeführt. Das Chlorid im Wasser-Ausfluss der Monolithe wird potentiometrisch bestimmt (siehe Kap.4.2.1).

Die Zeitintervalle der Probenahme werden so gewählt, dass komplette SolutDurchbruchskurven (DBK) erstellt werden können. Diese Durchbruchskurven werden in der gebräuchlichen Form als relative Konzentration (C/Co = Tracerkonzentration im Perkolat / Tracerkonzentration in der Tracerlösung) gegen die Austauschhäufigkeit des Porenvolumens aufgetragen (BIGGAR \& NIELSEN, 1962). Das Porenvolumen ist das wassergefüllte Porenvolumen, welches im gesättigten Zustand dem Gesamtporenvolumen (GPV) entspricht.

Um das Retentionsverhalten zu charakterisieren, sind besonders drei Punkte der DBK zu beachten: 1. Der Punkt, an dem das erste Solut durchbricht, 2. die Relativkonzentration des Tracers nach Abfluss eines wassergefüllten Porenvolumens und 3. die verlagerten wassergefüllten Porenvolumina, die benötigt werden, um eine Relativkonzentration von 0,9 zu erreichen. Die erste Zahl gibt Aufschluss über den präferenziellen Fluss, die zweite kann im Vergleich zum piston-flow-Modell ( $\mathrm{C} / \mathrm{Co}=0,5$ bei $1 \mathrm{PV})$ betrachtet werden und die dritte gibt Auskunft über die Schnelligkeit der langsamen Stoffbewegung im Boden.

Der Versuch endet erst, wenn die Konzentration im Ausfluss der Konzentration im Zulauf entspricht. Die zu diesem Zeitpunkt im Monolithen aufgenommene 
Chloridmenge (mg) kann als Porenvolumen (\%-Vol.) der Monolithe ausgedrückt werden, welches komplett mit Tracerlösung (1000 $\left.\mathrm{mg}^{-1} \mathrm{~L}^{-1}\right)$ ausgetauscht worden ist $\left(P V_{C l}\right)$ :

$$
\begin{aligned}
& P V_{C l}=\frac{\left(C_{O}\left[m g \cdot L^{-1}\right] \cdot O[L]\right)-\left(C_{A}\left[m g \cdot L^{-1}\right] \cdot A[L]\right)}{G V[L] \cdot C_{O}\left[m g \cdot L^{-1}\right]} \times 100[\%] \\
& \text { Co }=\text { zugeführte Tracerkonzentration }\left[\mathrm{mg}^{-1} \mathrm{~L}^{-1}\right] \\
& \mathrm{C}_{\mathrm{A}}=\text { Tracerkonzentration im Ausfluss }\left[\mathrm{mg}^{-1} \mathrm{~L}^{-1}\right] \\
& \mathrm{O}=\text { zugeführte Wassermenge [L] } \\
& \text { A } \quad=\quad \text { Ausflussmenge [L] } \\
& \text { GV = Gesamtvolumen des Lysimeters [L] }
\end{aligned}
$$

Mit dieser Methode ist es möglich, Unterschiede im austauschbaren Porenvolumen zwischen den verschiedenen Lagen herauszufinden. Diese ergeben zusammen mit der gesättigten Wasserleitfähigkeit wichtige bodenhydrologische Grunddaten für die Pelosole aus Röt.

Nicht der gesamte Porenraum des Bodens ist durch Chlorid austauschbar. Die Differenz aus Gesamtporenvolumen und chloridaustauschbarem Porenvolumen ergibt ein mit Wasser gefüllt Porenvolumen, welches jedoch für Solute nicht zugänglich ist. Man spricht hierbei von Anionenausschluss (BoND et al., 1984; SMILES et al., 1982). Es ist das Wasser der diffusen Doppelschicht auf den Tonteilchen, welches von $\mathrm{Cl}^{-}-$Ionen nicht erreicht werden kann.

\subsubsection{Zur Abschätzung der ungesättigten Wasserleitfähigkeit}

Auch im feldgesättigten Zustand ist der Boden nie oder nur vorübergehend vollständig wassergesättigt. Deshalb ist die Funktion der ungesättigten Wasserleitfähigkeit relevant, zu deren Abschätzung mehrere Methoden existieren (z.B. ARYA, 1976; Schindler, 1980; Plagge, 1991 ; Wendroth, 1990). Alle Methoden haben gemeinsam, dass im Bodenkörper ein Wasserspannungsgradient angelegt wird. Aus dem Verlauf des Wasserspannungsgradienten wird mit der Bodenfeuchte dann die ungesättigte Wasserleitfähigkeitsfunktion $\left(k_{u}\right)$ abgeleitet.

Die ungesättigte Wasserleitfähigkeit $\left(k_{u}\right)$ des Bodens ist von WENDROTH (1990) mit der langsamen Verdunstungsmethode für den gleichen Standort bestimmt worden. Dabei ist die Messung an den Varianten Acker, Grasland und Wald an 
$300 \mathrm{~cm}^{3}$ Stechzylindern erfolgt. Die Graslandvariante war von einem Grünstreifen neben dem Ackerland entnommen worden.

Zum Vergleich zu unseren Messungen ist folgendes zu beachten: Die Werte der hydraulischen Leitfähigkeit der Makroporen aus Stechzylindern sind nicht in einen größeren Maßstab übertragbar (MESSING \& JARVIS, 1995). Für die Matrix ist diese Übertragung möglich, da in einem Stechzylindervolumen von $300 \mathrm{~cm}^{3}$ die Strukturen der Matrixporen abgebildet werden können. Deshalb soll hier der Bereich der ungesättigten Leitfähigkeit nahe der Sättigung, also bei niedrigen Saugspannungen abgeschätzt werden, da die Makroporen bei der Stechzylindermethode nach WENDROTH (1990) nicht gut abgebildet werden und die hydraulische Leitfähigkeit in diesem Bereich unterschätzt wird.

Das Problem bei dieser Methode ist, dass der betrachtete Bodenausschnitt (Bodenmonolith) jeweils in sich homogen sein muss. Aus den vorangegangenen Versuchen ist bekannt, dass dies in den Versuchslysimetern nicht der Fall ist, bis auf das Lysimeter 1, welches nur Oberbodenmaterial ohne Solifluktionslage beinhaltet. Durch die Solifluktionslage in den längeren Lysimetern wird auch der Ausfluss des darüberliegenden Bodens beeinflusst.

Eine andere Methode zur Abschätzung der ungesättigten hydraulischen Leitfähigkeit des Bodens ist die Ausflussmethode (Outflow-method), die auf GARDNER (1956) zurückgeht und vielfach angewendet und modifiziert worden ist (z. B. VEREECKEN et al., 1997; VAN DAM et al., 1992; VAN DAM et al., 1994; STOLTE et al., 1994; TOORMAN et al., 1992; HOPMANS et al., 1992; VEREECKEN et al., 1990; BOHNE et al., 1993).

Der Wasserspiegel in den Monolithen befindet sich zu Beginn an der Bodenoberfläche. Ein Ausflussversuch an den Lysimetern 1, 2 und 4 wird vorgenommen, wobei der Wasserspiegel jeweils in $10 \mathrm{~cm}$ - Schritten schlagartig abgesenkt wird. Für den $10 \mathrm{~cm}$-Abschnitt des Bodens bedeutet dies, eine mittlere Saugspannung von $5 \mathrm{~cm}$ WS, also einen pF-Wert von 0,7 , bei dem die Poren mit einem Äquivalentdurchmesser von $600 \mu \mathrm{m}$ entwässert werden. Beim nächsten Absenkungsschritt werden zusätzlich zu den $600 \mu \mathrm{m}$ Poren der nächsten $10 \mathrm{~cm}$ die Poren bis $200 \mu \mathrm{m}$ der ersten $10 \mathrm{~cm}$ des Bodens entwässert. 
Nach dem Absenkungsversuch wird der Wasserspiegel im Lysimeter in der Absenkungstiefe konstant gehalten und so lange gewartet, bis das Restporenwasser der teilentwässerten Poren des betrachteten Lysimeterabschnitts ausgeflossen ist. Die Absenkungstiefen von der Bodenoberfläche sind in Tabelle 12 aufgeführt. Anschließend werden die Monolithe in $5 \mathrm{~cm}$ Tiefenabschnitten in Scheiben zerlegt. Jede Scheibe wird im feuchten Zustand gewogen und anschließend bei $105^{\circ} \mathrm{C}$ getrocknet und wieder gewogen (Trockensubstanz). Damit wird der gravimetrische Wassergehalt bestimmt. Mit den Trockensubstanzwerten wird mit Hilfe der Festsubstanzdichte $\left(d_{F}\right)$ die Trockendichte des Bodens $\left(d_{B}\right)$ und das Gesamtporenvolumen (GPV) errechnet:

$$
G P V=\left(\frac{d_{B}}{d_{F}}-1\right) \cdot 100[\%]
$$

Da die Lysimeter eine unterschiedliche Länge und hydraulische Druckhöhe haben, stellen sich in den einzelnen Tiefenabschnitten unterschiedliche Saugspannungen ein. Wenn wir davon ausgehen, dass jedes Lysimeter im selben Tiefenabschnitt die gleiche Porengrößenverteilung hat, können auf diese Weise die Wassergehalte im Oberboden von pF-Werten 0 bis 1,6 bestimmt werden. In Tabelle 8 sind die dabei entwässerten Äquivalentporendurchmesserbereiche in $10 \mathrm{~cm}$ Bodenabschnitten aufgetragen.

Tabelle 12: $\quad$ Berechnete Werte entwässerter Äquivalentporenbereiche (ÄPB) in Abhängigkeit von der Absenkungstiefe von der Oberfläche $(A T)$ in einzelnen Bodentiefenabschnitten von vier Lysimetern (Lysim.)

Lysim.- Lysim.- AT ÄPB $(\mu \mathrm{m})$ in verschiedenen Tiefenabschnitten $(\mathrm{cm})$

Nr. länge

\begin{tabular}{rccrrrrrr} 
& $(\mathrm{cm})$ & $(\mathrm{cm})$ & $0-10$ & $10-20$ & $20-30$ & $30-40$ & $40-50$ & $50-60$ \\
\hline 1 & 30 & 25 & $>150$ & $>300$ & & & & \\
4 & 31 & 30 & $150-120$ & $300-200$ & $>600$ & & & \\
2 & 53,1 & 50 & $120-67$ & $200-86$ & $600-120$ & $>200$ & $>600$ & \\
3 & 69 & 65 & $67-50$ & $86-60$ & $120-75$ & $200-100$ & $600-150$ & $>300$
\end{tabular}


Die Porenbereiche werden durch Differenzbildung errechnet. Man beginnt mit dem ermittelten Volumenanteil vom kleinsten Äquivalentporendurchmesser und kann diesen Volumenanteil dann vom nächst größeren Äquivalentporendurchmesser abziehen. So erhält man durch Differenzbildung einzelne Porenanteile für verschiedene Porengrößenklassen aus verschiedenen Lysimetern.

Nach Tabelle 12 können unter der Annahme, dass die Monolithe untereinander homogen sind, im Tiefenabschnitt 0-10 cm Bodentiefe die Volumenanteile der Poren mit Äquivalentdurchmessern von >150, 150 bis120, 120 bis 67 und 67 bis $50 \mu \mathrm{m}$ bestimmt werden. Diese Porenvolumen sollen dann mit den anderen Daten der ungesättigten hydraulischen Leitfähigkeit in Beziehung gebracht werden.

Abschließend wird aus dem getrockneten Boden der Skelettanteil (> $2000 \mu \mathrm{m}$ Durchmesser) durch Nasssiebung für die einzelnen Tiefenabschnitte bestimmt.

\subsection{Ergebnisse und Diskussion}

\subsubsection{Gesättigte Wasserleitfähigkeit}

Die gesättigten Leitfähigkeiten für die Lysimeter sind in Tabelle 13 aufgetragen. Mit zunehmender Lysimeterlänge sinkt die hydraulische Leitfähigkeit der Lysimeter. Dies spricht dafür, dass die Solifluktionslage eine deutlich niedrigere hydraulische Leitfähigkeit besitzt als der darüberliegende Boden.

Die Werte sind nach Bodenkundlicher Kartieranleitung (AG BODEN, 1994) für das Lysimeter 3 als sehr hoch bzw. für alle anderen Lysimeter als äußerst hoch einzustufen. Die Bodenart schwach schluffiger Ton (Tu2) und mittel schluffiger Ton (Tu3) lässt nur geringe bis mittlere hydraulische Leitfähigkeitswerte erwarten. Die für diese Einstufung verwendete standardmäßige $\mathrm{k}_{\mathbf{s}}$-Bestimmung erfolgte an Stechzylindern, bei denen Makroporen, wie Regenwurmgänge gemieden werden. Die Lysimeter enthalten hingegen einige Regenwurmgänge, die zur Erklärung der hohen hydraulischen Leitfähigkeit herangezogen werden können. Die Werte liegen in der Größenordnung der von DE MOL (1996) gefundenen gesättigten Infiltrometerwerten. Die von WENDROTH (1990) unter Gras-

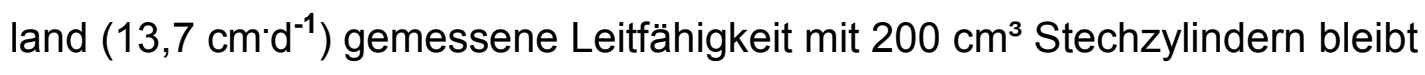
weit hinter den hier bestimmten Werten zurück. 
Tabelle 13: Gesättigte hydraulische Leitfähigkeiten bei Pelosol-Lysimetern mit und ohne Solifluktionslage

\begin{tabular}{cccc}
\hline Lysimeternummer & Lysimeterlänge $(\mathrm{cm})$ & $\mathrm{k}_{\mathbf{s}}\left(\mathrm{cm}^{-1} \mathbf{s}^{-1}\right)$ & $\mathrm{k}_{\mathbf{s}}\left(\mathrm{cm}^{\prime} \mathbf{d}^{-1}\right)$ \\
\hline 1 & 30,0 & $9,19 \cdot 10^{-2}$ & 7940,2 \\
4 & 31,5 & $4,04 \cdot 10^{-2}$ & 3490,3 \\
2 & 53,1 & $2,64 \cdot 10^{-2}$ & 2278,1 \\
3 & 67,5 & $5,19 \cdot 10^{-4}$ & 44,9 \\
\hline
\end{tabular}

Die Lysimeter 1 und 4 unterscheiden sich in der Wasserleitfähigkeit stark voneinander, obwohl sie sich in der Länge nur geringfügig voneinander unterscheiden. HARTGE (1994) zeigte, dass Stechzylinder mit einem Volumen von $346 \mathrm{~cm}^{3}$ in aggregierten Tonböden keine reproduzierbaren Werte liefern, da die Makroporen zu stark variieren. Diese Variation von wasserleitenden Poren, besonders von Makroporen, wie Regenwurmgängen, scheint sich auf einer Querschnittsfläche von $1075 \mathrm{~cm}^{2}$ auszuwirken. Lysimeter 1 hat im Gegensatz zu den anderen Lysimetern an der Oberfläche auffallend viele offene Regenwurmgänge, gezählt wurden 16 Stück mit einem Durchmesser von 0,13-0,9 cm. Solche Wurmgänge haben sehr hohe Kontinuitäten und bedingen die besonders hohen Leitfähigkeiten.

Die Größenordnung der gesättigten Leitfähigkeit erklärt, dass im Freiland zu keinem Zeitpunkt Oberflächenwasser auftritt. Auch im gequollenem Zustand ist die Leitfähigkeit außerordentlich hoch. Folglich ist auch die Austragsgefährdung für gelöste Stoffe hoch.

\subsubsection{Soluttransport}

Die Transportparameter für den Soluttransport werden im gesättigten Zustand ermittelt. In Abbildung 9 ist die Chlorid-Konzentration gegen die aus den Lysimetern ausfließende Wassermenge aufgetragen. Zu sehen ist der Zusammenhang zwischen Lysimeterlänge und Chloriddurchbruch.

Der Konzentrationsanstieg erfolgt bei allen Kurven anfänglich sehr steil und nähert sich dann asymptotisch dem Endwert. Eine Besonderheit ist in den Kurvenabschnitten zu sehen, bei denen die Konzentration zeitweise im Versuchsablauf abnimmt. Diese Abnahme ist bei Lysimeter 1 zwischen 0 und $50 \mathrm{~mm}$ undeutlich, bei den Lysimetern 2 und 3 zwischen 300 und 450 mm sehr deutlich (Abbildung 9) zu sehen. Diese beobachteten Konzentrationsverläufe lassen 
sich nur durch Lufteinschlüsse erklären. Auch beim langsamen Aufsättigen von der Lysimeterbasis her wird Luft im Boden eingeschlossen. HASE \& MEYER (1969) beschreiben diesen Vorgang des Lufteinschlusses durch kapillare Aufsättigung von Stechzylindern in einem Lössboden in den Poren mit dem Äquivalentdurchmesser von $3-8,5 \mu \mathrm{m}$.

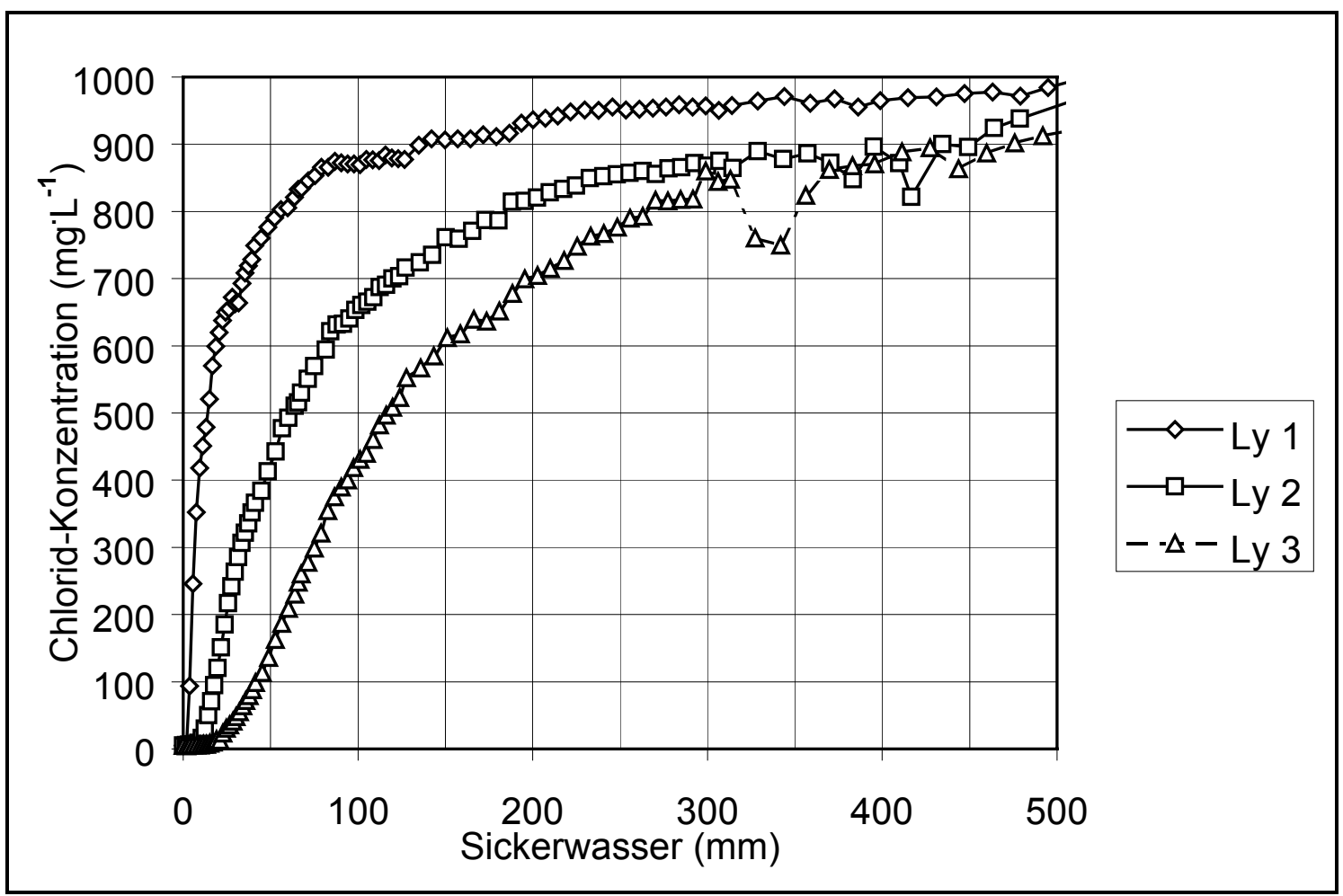

Abbildung 9: Chlorid-Austragskurve: Chlorid-Konzentration $\left(\mathrm{mg}^{-L^{-1}}\right)$ im Sickerwasser [mm] aus Pelosol-Lysimetern mit unterschiedlichen Längen ( Ly $1=30 \mathrm{~cm}$, Ly $2=53,1 \mathrm{~cm}$, Ly $3=67,5 \mathrm{~cm}$ ) bei dauerhafter Chlorid-Applikation (1000 $\mathrm{mg}^{-1} \mathrm{~L}^{-1}$ ) bei gesättigtem Fluss von $15 \mathrm{~mm}^{-1}$

Bei Stechzylindern versucht man diese Lufteinschlüsse durch die Aufsättigung mit Wasser unter Vakuum zu vermeiden. Für die Lysimeter ist dies aufgrund der Größe der Gefäße nicht möglich gewesen. Diese Luftblasen lösen sich im Versuchsablauf spontan vom Boden und entweichen nach oben. Dadurch kommt Bodenlösung, die vorher durch die Luft vom Tracer abgetrennt worden ist, in Kontakt mit der Tracerlösung und wird in relativ kurzer Zeit ausgetauscht. So kommt es zu einem mehr oder weniger starken Absenken der Konzentration im Ausfluss. 


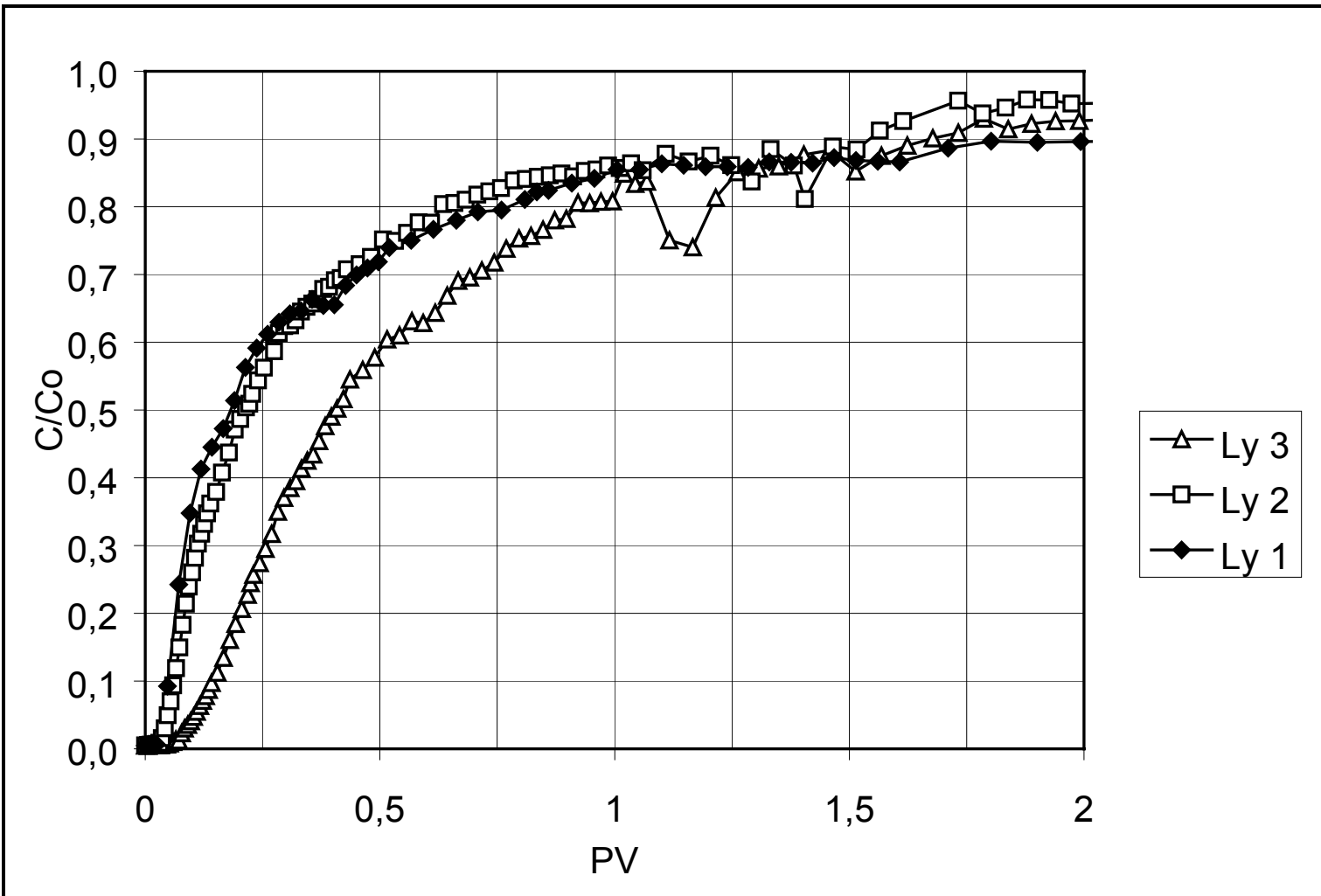

Abbildung 10: Chlorid-Durchbruchskurven: $C / C o=$ relative Konzentration; $P V=$ Perkolat der Lysimeter ausgedrückt in Gesamtporenvolumen (Lysimeterlängen: Ly $1=30 \mathrm{~cm}$, Ly $2=53,1 \mathrm{~cm}$, Ly $3=67,5 \mathrm{~cm}$ )

Weiterhin lässt sich aus den Konzentrationskurven der Lysimeter folgendes ableiten: Mit steigender Lysimeterlänge kommt der Chloriddurchbruch erst später im Sickerwasserausfluss an. Die Konzentration von $500 \mathrm{mg} \mathrm{L}^{-1}$ ist bei Lysimeter 1 nach 15, bei Lysimeter 2 nach 64, bei Lysimeter 3 nach $120 \mathrm{~mm}$ überschritten. Das bedeutet, dass die aufgegebene Tracermenge, die zur gleichen Konzentrationserhöhung führt, sich nicht linear zur Lysimeterlänge verhält. Deshalb wird die gebräuchliche Darstellung als Durchbruchskurve (BIGGAR \& NiELSEN, 1962), abgekürzt DBK, verwendet (Abbildung 10).

Zur Bewertung und Einordnung der DBK sind die Retentionseigenschaften in Tabelle 14 aufgeführt: Der erste Solutdurchbruch ist nach 0,02 bis 0,05 PV zu finden. Die relative Konzentration von 0,9 wird zwischen 1,2 bis 2 PV erreicht. Das bedeutet, dass ein stark präferenzieller, d.h. gegenüber dem „piston-flow“Modell ein bevorzugter Fluss vorherrscht. Die Endkonzentration wird erst nach 153 Tagen bzw. 5,6 bis 29,1 ausgetauschten Porenvolumen erreicht. Nach dem „piston-flow“-Modell hätte die Endkonzentration bereits nach einem Porenvolu- 
men erreicht werden müssen. Der Teil der DBK zwischen 1 PV und dem Erreichen der Endkonzentration wird als „tailing“ bezeichnet. Dieses „tailing“ ist gemessen an anderen miscible-displacement-Versuchen sehr ausgeprägt.

Tabelle 14: Retentionsparameter der Bodenmonolithe: GPV = Gesamtporenvolumen (\%-Vol.); PV $\left(\mathrm{Cl}^{-}\right)=$durch Chlorid ausgetauschtes Porenvolumen (\%-Vol.); $P V_{\text {ges }}=$ Anzahl durchflossener Porenvolumen; 1.DB = erster messbarer Solut-Durchbruch

\begin{tabular}{rrrrrrr}
\hline Lysimeter & GPV & PV $\left(\mathrm{Cl}^{-}\right)$ & $\mathrm{PV}_{\text {ges }}$ & $\begin{array}{r}\mathrm{C} / \mathrm{Co}=0,5 \\
(\mathrm{PV})\end{array}$ & $\begin{array}{r}\mathrm{C} / \mathrm{Co}=0,9 \\
(\mathrm{PV})\end{array}$ & 1.DB (PV) \\
\hline 1 & 41,9 & 25,0 & 29,1 & 0,2 & 2,2 & 0,02 \\
4 & 38,5 & & & & & \\
2 & 41,9 & 30,1 & 5,6 & 0,2 & 1,1 & 0,01 \\
3 & 40,5 & 29,8 & 5,8 & 0,3 & 1,2 & 0,03 \\
\hline
\end{tabular}

Zusammen mit dem präferenziellen Fluss sind die Parameter für den Soluttransport folgendermaßen zu charakterisieren: Das „tailing“ der Austragskurve ist auf diffusive Solutbewegung zurückzuführen. Dafür sind sogenannte Sackgassenporen (,dead-end-pores“) oder Diffusion in immobile Wasserbereiche verantwortlich (SCHLINDWEIN 1992). Der schnelle erste Durchbruch spricht in Pelosolen gegen Sackgassenporen, sondern für ein Makroporensystem mit hoher Kontinuität. Die langsame Annäherung an die Endkonzentration kommt durch die langsame Diffusion in die Bodenaggregate hinein zustande. Die aktive Wasserbewegung wird durch die hohe Aggregatdichte bis $1,9 \mathrm{~g} \mathrm{~cm}^{3}$ (Tabelle 7) behindert. Vom Prinzip her ist deshalb das hydrologische Verhalten dem einer Kalkstein-Rendzina ähnlich, da die Polyeder-Bodenaggregate sich hier hydrologisch wie die Steine der Rendzina verhalten. ScHULIN et al. (1984) geben für eine Kalkstein-Rendzina sogar eine höhere Retentionsleistung an, welche auf die vorhandenen Krümelaggregate zurückzuführen ist. KISSEL et al. (1973) haben unter gesättigten Bedingungen ähnliche Retentionsparameter in einem Tonboden gefunden, die Ergebnisse von TYLER \& THOMAS (1981) entsprechen auch im ungesättigten Zustand etwa den Parametern, die wir gefunden haben. BOUMA \& ANDERSON (1977) zeigten an gestörten Bodenzylindern, dass das „piston-flow“-Modell näherungsweise auf Tonböden ohne Sekundärporen an- 
gewendet werden kann, bei Proben mit einer künstlichen Makropore jedoch nicht.

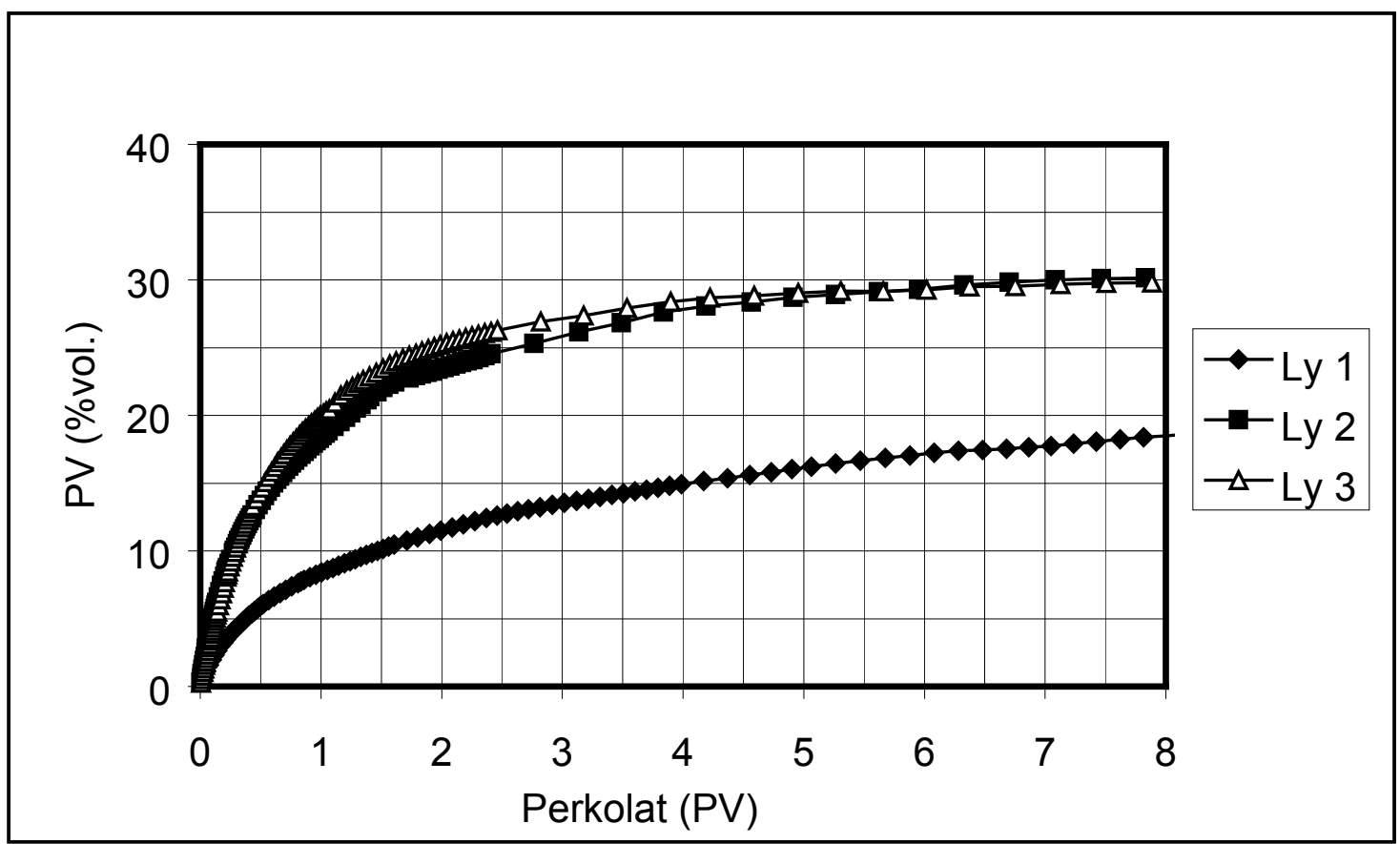

Abbildung 11: Verlauf der Chlorid-Aufnahme des Porenraumes (\%-vol.) in Abhängigkeit vom Perkolationsvolumen ausgedrückt als Porenvolumen der Lysimeter (\%-vol.) (Lysimeterlängen: Ly $1=30 \mathrm{~cm}, \mathrm{Ly}$ $2=53,1 \mathrm{~cm}$, Ly $3=67,5 \mathrm{~cm}$ ) berechnet nach Gleichung (6)

Bei unseren Monolithen ist die Diffusion in die Bodenaggregate davon abhängig, ob ein Monolith die Solifluktionslage enthält (Ly2 und 3) oder nicht (Ly1). Abbildung 11 zeigt die Chloridaufnahme in Abhängigkeit von der Perkolationsmenge. Es zeigt sich, dass nach einem durchflossenen Porenvolumen Ly 2 und 3 etwa $18 \%$-vol. und Ly 1 etwa 7 \%-vol. Chlorid ausgetauscht haben. Das bedeutet, dass die Solifluktionslage schneller Chlorid aufnehmen kann als das darüberliegende P-Horizontmaterial. Die Lysimeter mit Solifluktionslage nehmen auch in der Summe mehr Chlorid auf als das Lysimeter ohne. Das mit Chlorid austauschbare Porenvolumen ist also bei den Lysimetern mit Solifluktionslage größer, wenn auch die gesättigte Wasserleitfähigkeit niedriger ist.

Das lässt folgende Hypothese zu: Im Bereich der Solifluktionslagen muss eine Diskontinuität der Makroporen gegenüber dem Oberboden sein. Gleichzeitig ist die Diffusionsbewegung in immobile Bereiche des Bodens erhöht. Folglich 
muss die Solifluktionslage eine andere Porengrößenverteilung mit einem geringerem Maximum besitzen.

Es ist noch die Frage zu klären, ob solche gesättigten Versuche auch auf Feldbedingungen übertragen werden können oder ob es unter Feldbedingungen keinen präferenziellen Fluss gibt. Die Gefäßgröße ist nach dem bisherigen Kenntnisstand als gut geeignet zu beurteilen. Vielmehr stellt sich die Frage, ob die Höhe der Flussrate für Feldbedingungen zu hoch ist und nur durch die Höhe der Flussrate präferenzielle Flüsse zustande kommen, die es, möglicherweise, unter Feldbedingungen nicht gibt. Nach EDWARDS et al. (1979) kommt präferenzieller Fluss in Makroporen zustande, wenn die Flussrate in der Matrix und die Flussrate in die Matrix hinein überschritten wird. Im feldgesättigten Zustand ist die Flussrate in die Matrix gleich null, da es keinen Flussgradienten gibt. Die maximale Flussrate in der Matrix ist wegen der geringen Porendurchmesser auch sehr gering. Deshalb kann auch im feldgesättigten Zustand präferenzieller Fluss, wie auch in Kapitel 4.2.3 gezeigt, erwartet werden.

Ein größeres Problem für die Übertragung der Daten in die Fläche stellt die Variation des Porenraumes dar, welches im folgenden Kapitel untersucht werden soll.

\subsubsection{Zur Abschätzung der ungesättigten Wasserleitfähigkeit}

Die Funktion der ungesättigten Leitfähigkeit haben für Röt-Pelosole PLAGGE (1991) und WENDROTH (1991) erstellt. Es wurde aber weiter oben gezeigt, dass die absoluten Werte der gesättigten hydraulischen Leitfähigkeit von Stechzylindern sich von größeren Bodenmonolithen stark unterscheiden. Die ungesättigte Leitfähigkeitsfunktion ist an den Bodenmonolithen schwer zu messen.

Ausflussversuche mit einer plötzlichen Absenkung des Wasserspiegels um 10 cm ergeben folgende Erkenntnis: Bei den längeren Bodenmonolithen (Ly2 und 3) kann die Ausfluss-Methode nicht angewendet werden, da die geringe gesättigte Leitfähigkeit im unteren Lysimeterabschnitt stark hemmend auf den Abfluss wirkt und deshalb die Entwässerung der obersten $10 \mathrm{~cm}$ mehrere Tage benötigt, während die kurzen Lysimeter in der ersten Stunde etwa 30 ml Wasser abgeben. 
In Abbildung 12 ist die Ausfluss-Zeit-Funktion des 57,5 cm langen Bodenmonolithen abgebildet. Sie verhält sich grundsätzlich anders als die Funktionen in Abbildung 14, da die Zeitdauer für den gesamten Ausfluss sehr viel größer ist als bei den anderen. Dies ist auf den Einfluss der Solifluktionslage zurückzuführen.

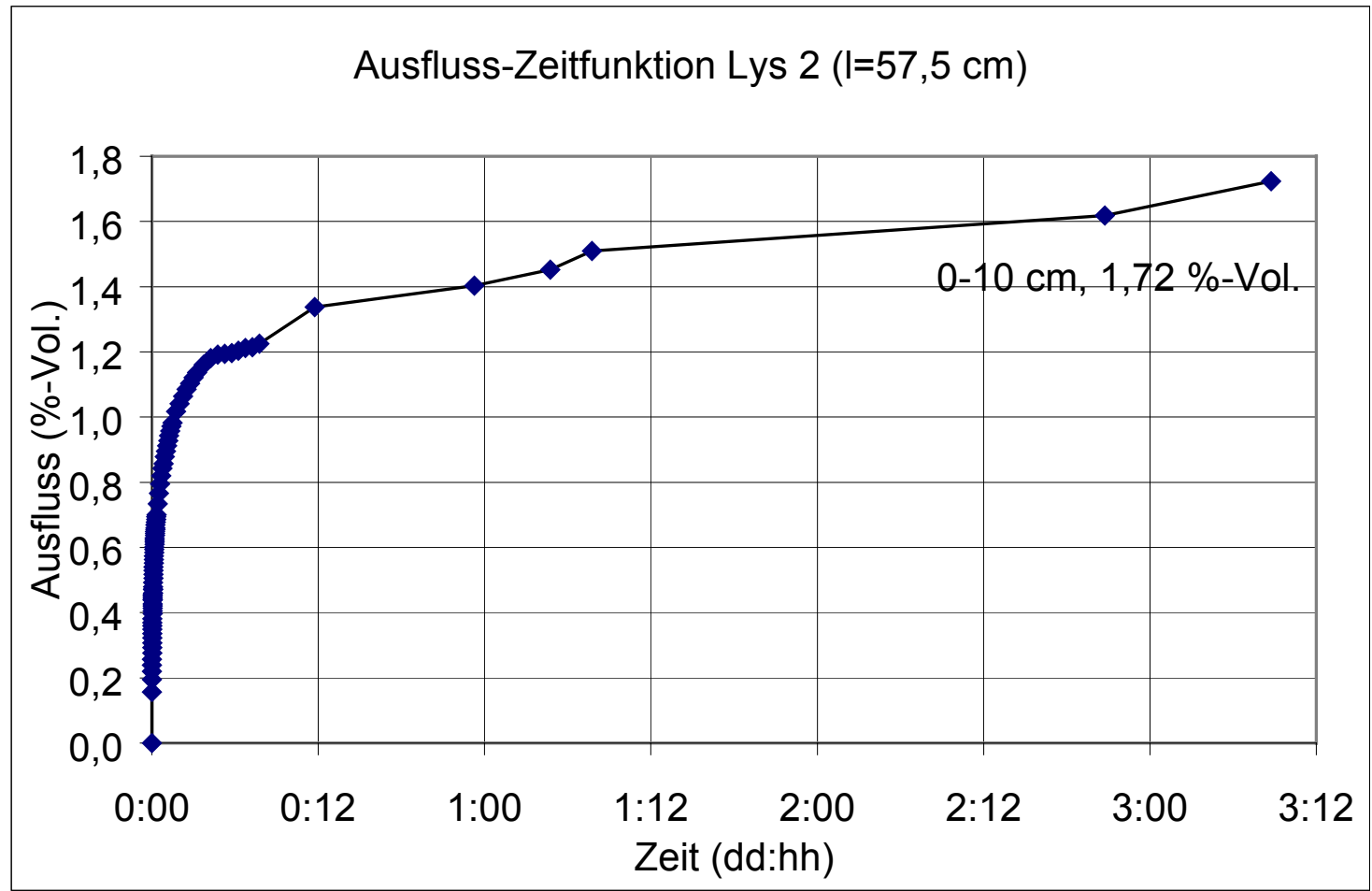

Abbildung 12: Ausfluss-Zeitfunktion aus den oberen $10 \mathrm{~cm}$ bei plötzlicher $A b$ senkung des Wasserspiegels bei dem $57,5 \mathrm{~cm}$ langen Bodenmonolith

Aus den Ausfluss-Zeitfunktionen der beiden kürzeren Monolithe (Abbildung 13) ist folgendes abzuleiten: Bei Lys 1 ist die Gesamtentwässerungsmenge im obersten Abschnitt am höchsten, die darunterliegenden Abschnitte weisen weniger Wasser auf. Erwartet wurde, dass bei der Entwässerung der unteren Abschnitte auch aus den oberen Abschnitten Wasser mit höherer Saugspannung nachfließt. Die Wassermenge, die aus dem oberen Abschnitt zusätzlich fließt, muss also sehr gering sein.

Das Porenvolumen von Lys 1, welches durch die Absenkung entwässert wird, nimmt von oben nach unten ab. Das höhere Grobporenvolumen in der obersten Schicht kann durch Wurzelgänge der Grasnarbe erklärt werden. Bei Lys 4 ist das entwässerte Porenvolumen in der untersten Schicht höher als in der darü- 
berliegenden, die Ausflussfunktion zeigt, dass das Wasser langsamer abfließt als aus den darüberliegenden Schichten.

Lys 1 und 4 unterscheiden sich im Ausflussverhalten und im Gesamtausflussvolumen in der Bodentiefe 20-28,5 bzw. 30 cm deutlich voneinander. Eine Bodenfläche von $0,1 \mathrm{~m}^{2}$ reicht also nicht aus, um die Porengrößenklasse $>600 \mu \mathrm{m}$ Äquivalentporendurchmesser repräsentativ zu erfassen. Die Berechnung der ungesättigten Leitfähigkeit in diesem Intervall nach GARDNER (1956) ergibt keine plausiblen Werte. Das kann nur darauf zurückzuführen sein, dass das GardnerKonzept auf der Darcy-Gleichung, also auf einem System mit laminarem Fluss, basiert. In Makroporen hingegen herrscht turbulenter Fluss (LOGSDON, 1995).

Die entwässerten Porenvolumen für den Tiefenabschnitt 0-10 cm liegen zwischen 1,42 und 1,72 \%-Vol. und somit in den gleichen Größenordnungen wie die von WENDROTH (1991) mitgeteilten Werten. Diese gelten für einen Äquivalentporendurchmesser von $600 \mu \mathrm{m}$. Bei Lys 1 wurden alle an der Oberfläche sichtbaren Regenwurmporen ausgemessen. Unter der Annahme, dass jede Pore einen senkrecht stehenden Zylinder bildet, wird das Porenvolumen errechnet. Es beträgt 0,25 \%-Vol. und kann nur einen Teil der 1,42 \%-Vol. erklären. Diese Regenwurmgänge konnten beim Zerstören der Bodenmonolithe zum Versuchsende verfolgt werden. Der andere Teil des Grobporensystems könnten Kluftporen sein, die während der Bodenbildung durch die P-Horizontbildung entstanden sind und visuell im Anschnitt nicht sichtbar sind. 


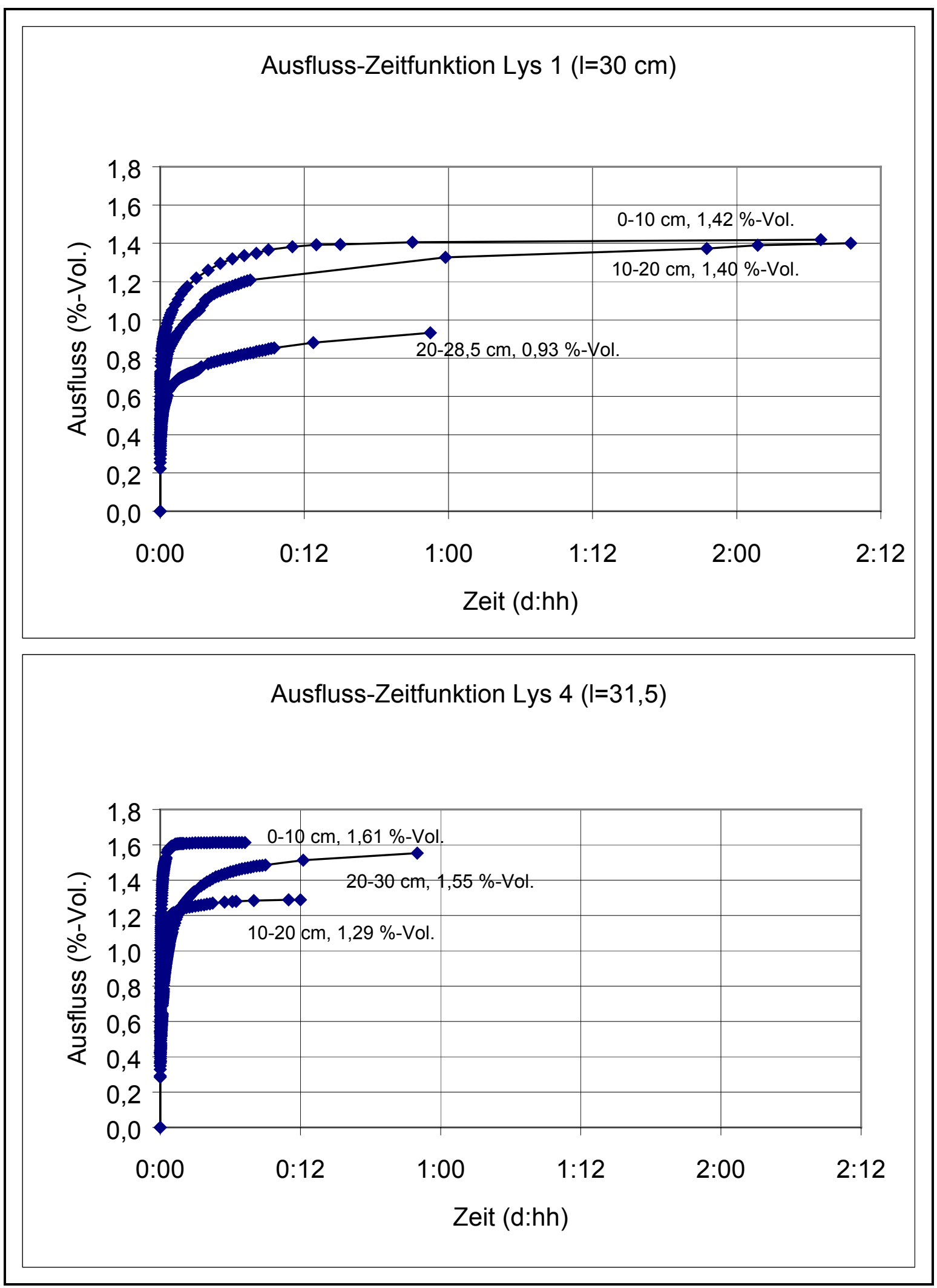

Abbildung 13: Ausfluss-Zeitfunktion aus Lysimetern bei plötzlicher Absenkung des Wasserspiegels um 10 bzw. $8,5 \mathrm{~cm}$ 
Das Gesamtporenvolumen und der Skelettanteil des Bodens liefern folgende Aussagen: Beim Gesamtporenvolumen (Tabelle 15) sind in den obersten $10 \mathrm{~cm}$ die größten Werte, meist über 40 \%-Vol, zu beobachten. Darunter geht das Gesamtporenvolumen zurück. In den Bodentiefen $>45 \mathrm{~cm}$ steigt das Gesamtporenvolumen wieder über Werte von 40 \%-Vol. an. Der Skelettgehalt (Tabelle 16) ist in den oberen $20 \mathrm{~cm}$ relativ einheitlich bei Werten um $4 \%-G e w$. , in den darunterliegenden Tiefenabschnitten sind größere Schwankungen von 0,7-20,2 \%-Gew. zu beobachten.

Tabelle 15: Gesamtporenvolumen (\%-Vol.) in Abhängigkeit von der Bodentiefe von 4 Bodenmonolithen unter Dauerbrache

\begin{tabular}{lllll}
\hline Bodentiefe $(\mathrm{cm})$ & Ly 1 & Ly 4 & Ly 2 & Ly 3 \\
\hline $0-5$ & 42,6 & 41,6 & 41,7 & 39,8 \\
$5-10$ & 45,3 & 44,0 & 42,5 & 42,0 \\
$10-15$ & 38,0 & 40,3 & 38,0 & 34,6 \\
$15-20$ & 41,9 & 22,6 & 40,2 & 35,8 \\
$20-25$ & 33,9 & 35,2 & 40,7 & 38,8 \\
$25-30$ & & 37,1 & 41,4 & 35,5 \\
$30-35$ & & 38,4 & 43,9 \\
$35-40$ & & & 42,4 & 37,3 \\
$40-45$ & & & 36,6 & 34,7 \\
$45-50$ & & & & 40,9 \\
$50-55$ & & & & 41,9 \\
$55-60$ & & & & 40,8 \\
$60-65$ & & & & 40,3 \\
\hline
\end{tabular}

Die Erklärung für die Veränderung der Gesamtporenvolumina und der Skelettanteile ist durch die Genese der Böden und des Gesteins selbst wie folgt darzustellen: Pelosole entstehen aus Pelitgestein. Das diagenetisch verfestigte Pelitgestein wirkt hydrologisch wie ein Kluftgestein, also hoch wasserdurchlässig. Mit zunehmender Verwitterung des Pelitgesteins nimmt die Wasserleitfähigkeit ab. Die feldgesättigte Wasserleitfähigkeit korreliert mit dem Skelettgehalt des Bodens. Der Skelettanteil des Bodens kann also als Indiz für eine hohe Wasserleitfähigkeit genommen werden, niedrige Skelettanteile sind mit niedrigeren Leitfähigkeiten verbunden. Die gesättigten Leitfähigkeiten unserer Bodenmonolithe sind hoch und in Abhängigkeit mit zunehmender Länge abnehmend. Die Monolithe, die einen Tiefenabschnitt mit niedrigem Skelettanteil haben, weisen 
auch eine geringe hydraulische Leitfähigkeit auf. Diese skelettarme Schicht wirkt als begrenzender Faktor auf die Leitfähigkeit.

Tabelle 16: Skelettanteil des Bodens (\%-Gew.) in Abhängigkeit von der Bodentiefe von 4 Bodenmonolithen unter Dauerbrache

\begin{tabular}{r|rrrr}
\hline Bodentiefe $(\mathrm{cm})$ & Ly 1 & Ly 4 & Ly 2 & Ly 3 \\
\hline $0-5$ & 4,7 & 4,4 & 4,0 & 4,1 \\
$5-10$ & 4,1 & 5,7 & 4,1 & 4,3 \\
$10-15$ & 4,3 & 5,0 & 5,3 & 4,0 \\
$15-20$ & 4,7 & 3,9 & 4,0 & 3,6 \\
$20-25$ & 4,5 & 4,5 & 0,9 & 0,7 \\
$25-30$ & & 0,9 & 2,1 & 0,7 \\
$30-35$ & & & 8,3 & 1,1 \\
$35-40$ & & & 8,7 & 5,6 \\
$40-45$ & & & 3,2 & 20,2 \\
$45-50$ & & & & 17,2 \\
$50-55$ & & & & 2,9 \\
$55-60$ & & & & 4,8 \\
$60-65$ & & & & 5,3 \\
\hline
\end{tabular}

In Tabelle 17 sind die Mittelwerte des Skelettanteils und das Gesamtporenvolumen der vier Bodenmonolithe in $5 \mathrm{~cm}$ Tiefenabschnitten aufgeführt. Im Gesamtporenvolumen unterscheiden sich die einzelnen Tiefenabschnitte nicht wesentlich voneinander. Die Gesamtporenvolumen nehmen mit der Bodentiefe ab. Diese Abnahme ist jedoch nicht signifikant. Der Skelettgehalt in 20-25 cm Bodentiefe unterscheidet sich signifikant $(p<0,05)$ von den Skelettgehalten der einzelnen Tiefenabschnitte in 0-20 cm. Die Variation der Skelettgehalte ist in den oberen $20 \mathrm{~cm}$ im Vergleich den größeren Bodentiefen gering.

Da die oberen $20 \mathrm{~cm}$ in früheren Jahren gepflügt worden sind, sind die Skelettanteile gleichmäßig verteilt. In den Bodentiefen darunter schwanken sie von 0,7 - 20,2 \%-Gew. Die hydrologischen Verhältnisse, die durch den Verwitterungsgrad des Pelitmaterials beeinflusst werden, variieren damit sehr stark. Die Trockendichten des Bodens schwanken zwischen 1,45 und 1,73, so dass die geringe Diffusion der Solute in die Aggregate hinein wegen der hohen Trockendichte und der geringen Teilchengröße erklärt werden kann. 
Tabelle 17: $\quad$ Mittelwerte und Variationskoeffizient (Vk.) vom Skelettanteil und Gesamtporenvolumen (GPV) von vier Bodenmonolithen in verschiedenen Tiefenabschnitten

\begin{tabular}{l|cc|cc}
\hline \multirow{2}{*}{ Bodentiefe (cm) } & \multicolumn{2}{|c|}{ Skelettanteil $(\%-G e w)}$. & \multicolumn{2}{c}{ GPV (\%-Vol.) } \\
\cline { 2 - 5 } & Mittelwert & Vk. & Mittelwert & Vk. \\
\hline $0-5$ & 4,3 & 7,51 & 41,4 & 2,79 \\
$5-10$ & 4,5 & 16,62 & $* * 43,5$ & 3,39 \\
$10-15$ & 4,6 & 12,92 & 37,7 & 6,16 \\
$15-20$ & 4,0 & 11,39 & $* * 35,1$ & 24,83 \\
$20-25$ & $* 2,6$ & 81,28 & 37,1 & 8,53 \\
\hline
\end{tabular}

$*=20-25 \mathrm{~cm}$ unterscheidet sich signifikant $(p<0,05)$ von allen anderen

${ }^{* *}=5-10$ und $15-20 \mathrm{~cm}$ unterscheiden sich signifikant $(p<0,05)$ voneinander

In Tabelle 18 sind die aus dem Absenkungsversuchs entwässerten Porenbereiche aufgetragen. Die Werte der ermittelten Porenvolumen müssen nach unserer Erwartung vom größeren Porendurchmesser zum kleineren Porendurchmesser hin zunehmen, da bei zunehmender Entwässerung der entwässerte Porenraum zunehmen muss. Das Ziel, für die verschiedenen Bodentiefenabschnitte einzelne Porengrößenbereiche zu ermitteln, ist gescheitert, da man durch Differenzbildung in einigen Fällen auch zu negativen Porenanteilen kommt. Hierfür gibt es zwei Erklärungsmöglichkeiten:

1. Die Variabilitäten der Porenanteile sind zwischen den einzelnen Lysimetern so groß, dass die Fläche der Lysimeter von $1075 \mathrm{~cm}^{2}$ nicht ausreicht, um alle Porengrößenklassen repräsentativ zu erfassen. Die Streuung einer Volumenanteile einer Porengrößenklasse ist größer als der Volumenanteil, der durch die nächst kleinere Porengröße hinzukommt.

2. Beim Entnehmen der Lysimeter im Freiland gab es die Schwierigkeit, die zylindrischen Bodenmonolithe sauber abzustechen. Durch den Skelettanteil wurde in bestimmten Bodentiefen mehr Material aus der Wandung herausgebrochen als ursprünglich geplant war. Es besteht die Möglichkeit, dass so einzelne Tiefenabschnitte einen etwas geringeren Durchmesser als $37 \mathrm{~cm}$ haben. Dies wirkt sich bei der Messung von Makroporen besonders stark aus, da ihr Volumenanteil sehr gering ist und eine Ungenauigkeit einen rela- 
tiv hohen Effekt auf den Messwert hat. Die Ermittlung der Gesamtporenvolumina werden hierdurch weniger beeinflusst.

Tabelle 18: Berechnete Werte entwässerter Äquivalentporenbereiche (ÄPB) und Messwerte des entwässerten Porenvolumens (PV) in Abhängigkeit von der Absenkungstiefe (AT) in einzelnen Bodentiefenabschnitten von vier Lysimetern (Zahl in Klammern ist die Absenkungstiefe des Wasserspiegels von der Bodenoberfläche)

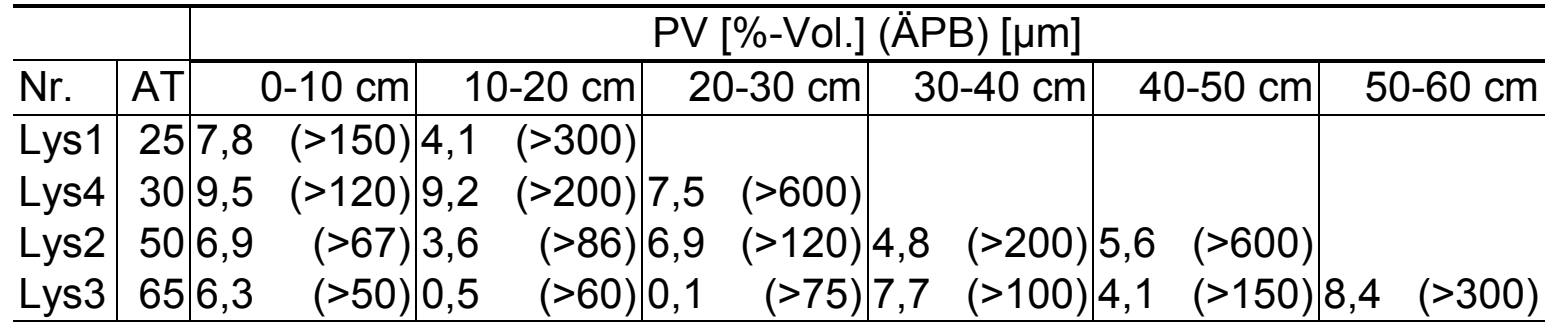

\subsection{Schlussfolgerungen aus den Laborlysimeterversuchen}

Die hydraulische Leitfähigkeit nimmt mit zunehmender Bodentiefe ab. Das Makroporensystem des Bodens besteht, neben einem Teil aus röhrenförmigen Regenwurmgängen, vor allem aus einem Kluftporensystem, welches nach unten hin abnimmt, mit der Folge einer nach unten abnehmenden Leitfähigkeit. Dadurch kommt es in Abhängigkeit von der Niederschlagshöhe zu unterschiedlichen Anteilen von vertikalen und lateralen präferenziellen Wasserflüssen. Die vertikalen Flüsse sind in den Transporteigenschaften hinreichend charakterisiert, die lateralen Flüsse, welche in Pelosolen typisch sind, können auf Basis der Lysimeterergebnisse noch nicht beschrieben werden. Die Anwendung von herkömmlichen Transportmodellen für die Feldskala scheidet durch die Variabilität der Stoffflüsse in räumlicher und zeitlicher Hinsicht aus. Die bisher angewendeten Verfahren zur Aufklärung der Porenstruktur (SPANGENBERG, 1996; DE MoL, 1996) können diese heterogenen Strukturen nicht abbilden.

Tonböden werden gemeinhin als gering auswaschungsgefährdet für gelöste Stoffe eingestuft. Die Versuche mit Chloridtracer haben gezeigt, dass die Pelosole eine hohe potentielle Auswaschungsgefährdung aufweisen, welche differenziert betrachtet werden muss: Das Retentionsvermögen für Solute ist gering, das Transportpotential ist, wie auch für partikuläre Substanzen (z.B. Bodenpar- 
tikel, Pflanzenschutzmittel) hoch. Der komplette Austausch der Bodenlösung vollzieht sich jedoch sehr langsam, da die Bewegung in den Bodenaggregaten fast nur durch Diffusion erfolgt. Eine besonders hohe Austragsgefährdung ergibt sich deshalb im feldgesättigten Zustand im zeitigen Frühjahr, wenn direkt nach der Ausbringung von Dünger (z.B. Nitrat) oder Pflanzenschutzmittel ein Regenereignis folgt. Die Matrix ist dann für den Stofftransport nur äußerst gering wirksam, und eine Verlagerung kann über schnellen Transport in den Makroporen erfolgen. Wenn der zeitliche Abstand zwischen Ausbringung und Niederschlagsereignis groß genug ist, dann kann das Solut in die Matrix diffundieren. Die Austragsgefahr ist dann gering, da die Diffusion aus der Matrix heraus nur stattfindet, solange die Makropore mit Wasser gefüllt ist. Dies kommt in der ungesättigten Bodenzone nur ausnahmsweise oder in Teilbereichen vor. In unseren Pelosolen ist dies in den Horizontbereichen mit Pseudogleymerkmalen möglich (vgl. Kapitel 3.4.2).

Die Pflanzenwurzel kann sich nur in Sekundärporen bewegen. Die Nährstoffausnutzung für gelöste Stoffe kann auch deshalb wegen der örtlich geringen Durchwurzelung der Bodenaggregate erschwert sein. Hinzu kommt eine hohe Aggregatdichte, die die Diffusion in der Matrix behindert.

Die Variabilität der Makroporen und des Verwitterungsgrades des Pelitmaterials ist äußerst hoch. Mit konventionellen Methoden, wie der Stechzylindermethode, sind repräsentative Messwerte nicht zu bekommen, da der Einzelausschnitt zu klein ist.

Die Solifluktionslage in den tieferen Bodentiefen hat bei der gesättigten hydraulischen Leitfähigkeit gegenüber dem P-Horizontmaterial einen hemmenden Einfluss. Der präferenzielle Fluss wird dadurch etwas verlangsamt. Der Austausch von Solut gegen Bodenlösung erfolgt in der Solifluktionslage schneller als im PHorizont. Die insgesamt ausgetauschte Solutmenge (Chlorid) ist in der Solifluktionslage höher als im P-Horizont, obwohl alle Monolithe das gleiche Gesamtporenvolumen haben. Die Solifluktionslage hat, wie erwähnt andere hydrologische Eigenschaften als der P-Horizont. Das Retentionsvermögen ist zwar etwas höher als beim P-Horizont, im Gelände muss jedoch berücksichtigt werden, dass die einzelne Solifluktionslagen ausschließlich für den Wassertransport verantwortlich sind und dadurch die Filterwirkung bei entsprechend hohen 
Fließgeschwindigkeiten gering sind. Je schlechter das Pelitmaterial verwittert ist, desto höher ist die hydraulische Leitfähigkeit. Ein Durchbruch des Sickerwassers, welches sich den bevorzugten Fließbahnen bewegt, in tiefere Bodenschichten und schließlich bis in den mittleren Buntsandstein hinein, kann nicht ausgeschlossen werden. Aus diesem Grunde sollen diese Solifluktionslagen anschließend näher betrachtet werden.

Die Beobachtung, dass es auch bei Starkregenereignissen in unserem Versuchsgebiet keine Anzeichen für nennenswerten Oberflächenabfluss gibt, kann durch die äußerst hohe hydraulische Leitfähigkeit der Bodenmonolithe erklärt werden. Innere Erosion konnte an einem Bodenmonolith demonstriert werden.

Es wird davon ausgegangen, dass auch unter Graslandvegetation ein Abschwemmen und Austragen von Bodenteilchen möglich ist. So können auch solche Standorte über den Zwischenabfluss und über Dränagen zur Gewässer Eutrophierung, z.B. durch an den Bodenpartikeln sorbiertes Phosphat, beitragen. 


\section{Aufnahme der Feinstratigraphie und boden- physikalischer Parameter}

Abschließend soll eine Vorstellung vom Stofftransport auf der Feldskala und der dazugehörigen Bodenstrukturen gewonnen werden. Dies geschieht anhand einer großen Profilaufnahme im Gelände.

\subsection{Methodik}

\subsubsection{Profilansprache}

Es wird ein $18 \mathrm{~m}$ langer und ca. 1,5 m breiter und $2 \mathrm{~m}$ tiefer Profilgraben angelegt, an dessen Profilwand der Boden hinsichtlich Bodenfarbe (MUNSELL SOIL COLOR CHARTS, 1975), freiem Carbonat, Struktur und Horizontierung angesprochen wird.

Das Profil wird fast senkrecht zum Verlauf des Lysimeterkellers der Plattenlysimeteranlage angelegt, damit für das Verständnis der Lysimeterversuche die lateralen Fließstrukturen von der Seite her studiert werden können. Die Lage des Profilgrabens ist in Abbildung 14 zu sehen. Der Profilgraben verläuft in Richtung SSW nach NNE. In Kapitel 4.2 wurde gezeigt, dass die präferenziellen, lateralen Flüsse auf der Versuchsfläche von einzelnen Solifluktionslagen abhängen. Damit man einen räumlichen Eindruck gewinnen kann, wird der Verlauf und das Gefälle der Solifluktionslagen gezeichnet. Senkrecht zur Lage des Grabens wird das Einfallen der Solifluktionslagen vermessen. Mit Salzsäure werden die Bodenabschnitte, die freies Carbonat aufweisen, sichtbar gemacht und in die Zeichnung aufgenommen. Mit Hilfe der Bodenfarbe und des sichtbaren Gefüges wird das Profil angesprochen. Aus dem Bodenprofil werden zwei vertikale Teilprofile ausgewählt, die sich nach der Geländeansprache wesentlich in den visuellen Eigenschaften und im Verwitterungsgrad unterscheiden. Das Vertikalprofil mit stark verwitterten, umgelagerten Pelitmaterial wird als Pelosol-Akkumulationsprofil $(P)$, das weniger stark verwitterte Pelitmaterial als Verwitterungsprofil (Cv) bezeichnet. Diese zwei Profile werden dann im folgenden näher untersucht und die einzelnen Horizonte genauer beschrieben. 


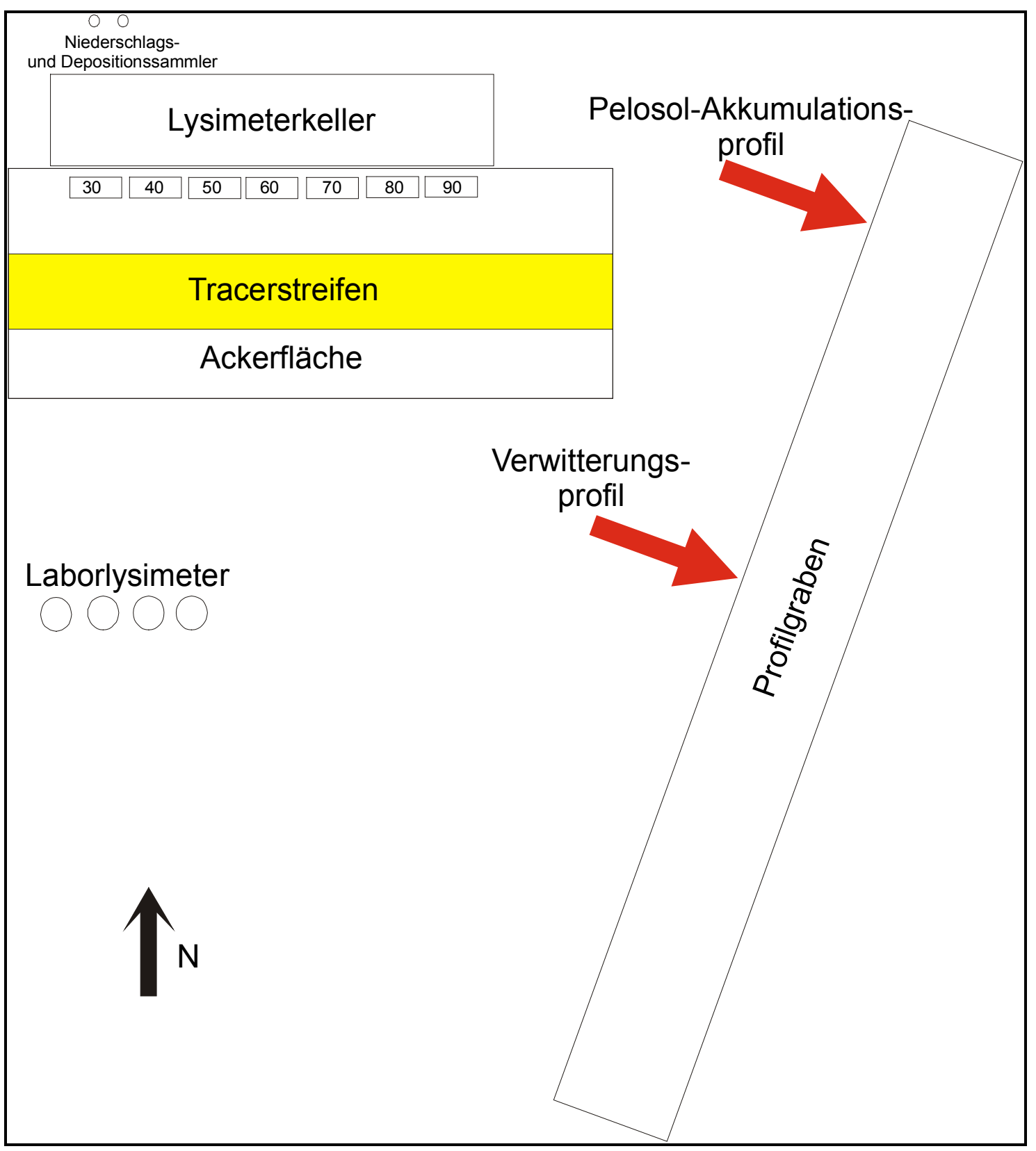

Abbildung 14: Lageskizze der Feldversuche am Kleinen Knüll bei Reinhausen 1997/98 (3 \% Hangneigung nach Süden)

\subsubsection{Bodenphysikalische Parameter}

\subsubsection{Trockendichten}

Von zwei Profilen wird von 0-200 cm Tiefe im Abstand von $20 \mathrm{~cm}$ die Trockendichte bestimmt. Die Dichteproben haben ein Volumen von $732 \mathrm{~cm}^{3}$. Zur Bestimmung der Trockendichte werden die Proben bei $105^{\circ} \mathrm{C}$ bis zur Gewichtskonstanz getrocknet und dann die Trockensubstanz gewogen und auf das Volumen bezogen. 


\subsubsection{Wassergehalts-Saugspannungsbeziehung}

Die Stechzylinder für die Erstellung der pF-Charakteristik haben ein Volumen von 981 bzw. $354 \mathrm{~cm}^{3}$. Die Höhe der eingestellten Saugspannung wird auf die Stechzylindermitte bezogen. Die Höhe der Stechzylinder beträgt 12 bzw. $5 \mathrm{~cm}$, die Bezugshöhe des hydrologischen Gradienten beträgt demnach 6 bzw.

$2,5 \mathrm{~cm}$. Aus diesem Grunde unterscheiden sich die pF-Werte in den unteren Druckstufen bei gleicher angelegter Saugspannung geringfügig um 3,5 cm WS Saugspannung. Bestimmt wird die Desorptionskurve im Drucktopf. Die Proben werden vollständig mit Wasser aufgesättigt, anschließend bestimmt man die pF-Werte bei abnehmenden Wassergehalten. Nach Erreichen der pF-Stufe 3 wird die vertikale Mächtigkeit der Probe auf $4 \mathrm{~cm}$ verkürzt, da der Zeitraum bis zum Erreichen des Gleichgewichtspunktes zu zeitaufwendig wäre. Die pFStufen 3,7 und 4,2 werden durch Herstellung einer Paste aus dem restlichen Stechzylindermaterial im Hochdrucktopf parallel erstellt.

\subsubsection{Korngrößenanalyse}

Von den Stechzylinderproben und von einem Teilprofil werden Korngrößenanalysen erstellt. Die angewendete Methodik der Korngrößenanalyse nach ATterberg (Schlichting et al., 1995) ist die kombinierte Sieb- und Sedimentationsanalyse. Diese Methode wird auf zwei verschiedene Weisen durchgeführt:

Beim ersten Verfahren nach DIN 19683 werden 20 g Boden mit 300 ml destilliertem Wasser versetzt. Nach dem vorher separat bestimmten Carbonatgehalt wird die äquivalente Menge Salzsäure hinzugegeben. Dazu werden $1 \mathrm{~g}$ Natriumdithionit, $5 \mathrm{ml}$ Natriumhydroxid (1,25 \%-Gew.) und $30 \mathrm{ml}$ Natriumcitrat gegeben und anschließend wird die Probe 3 Tage bei $80^{\circ} \mathrm{C}$ in den Trockenschrank gestellt. Die Kittsubstanzen, die die Primärteilchen des Pelites zusammenhalten, werden vollständig zerstört.

Das zweite, etwas modifizierte Verfahren arbeitet ohne Zerstörung der Kittsubstanzen. Dazu werden $20 \mathrm{~g}$ trockener Boden mit $300 \mathrm{ml}$ destilliertem Wasser und $20 \mathrm{ml}$ Ammoniaklösung versetzt. Die Proben werden anschließend 6 Stunden geschüttelt. Dadurch sollen die Bodenteilchen dispergiert werden, ohne die Kittsubstanzen zu zerstören.

Während der P-Horizontbildung im Periglazialzeitraum sind die Kittsubstanzen aufgebrochen worden. Im Gelände gibt es jedoch lokale Bereiche, in denen die 
Kittsubstanzen nicht vollständig zerstört sind und das Pelitausgangsgestein nicht vollständig aufgearbeitet wurde. Diese Bereiche geben im Gelände bei der Fingerprobe den Eindruck, dass es sich um schluffhaltiges oder feinsandhaltiges Material handelt. Da im Feld diese Körnung hydrologisch wirksam sein kann, wird daher bei der zweiten Korngrößenanalyse die Kittsubstanz nicht zerstört. Die Ergebnisse werden daraufhin überprüft, welche Korngrößenanalyse am besten die hydrologischen Eigenschaften wie den Totwassergehalt wiedergibt.

Die hier in den Korngrößenanalysen ermittelten Sandgehalte sind Pseudosande, da es sich um verbackene Tonteilchen handelt. Diese Pseudosande werden mit den Carbonatgehalten im Verwitterungsprofil in Beziehung gebracht um festzustellen, ob einer der Parameter von der Bodentiefe abhängig ist.

\subsection{Ergebnisse der Feinstratigraphie}

\subsubsection{Aufbau des Gesamtprofils}

In Abbildung 15 ist eine Skizze des Profilgrabens mit Blick nach Westen zu sehen. Die einzelnen Solifluktionslagen, die farblich gut zu unterscheiden sind, bilden einen Sattel. An der rechten und an der linken Profilseite ist das Material bis in $2 \mathrm{~m}$ Bodentiefe homogenisiert, nach Fingerprobe stärker tonhaltig als in der Mitte des Profils. Links und rechts neben dem Sattel schließt sich laminare Solifluktion an, die besonders gut an der linken Seite an den ausgedünnten carbonathaltigen Bändern zu erkennen ist. Der Sattel in der Profilmitte scheint durch Solifluktion kaum bewegt worden zu sein. Er ist nur ein Stück, auf der Skizze nach links, den Hang abwärts geflossen, was an der gedrückten Form des Eiskeiles in der Profilmitte zu sehen ist. Dies ist eine Teil des Kartenspielmodells, welches in Kapitel 3.1 näher beschrieben ist: Die tieferen Lagen sind nur gering verlagert worden, während der Oberboden schneller verlagert wird und über dem teilweise Sattel fehlt.

Anhand der Geländeform wurde die Sattelstruktur der Schichten im Untergrund, die nach Norden, also im Profil gegen die Geländesteigung einfällt, nicht erwartet. Die Sattelstruktur lässt den Schluss zu, dass an den beiden Seiten des Profils in größerer Bodentiefe Materialschwund stattgefunden hat und deshalb immer wieder durch das Periglazialklima das von der Oberfläche stammende, 
stärker verwitterte Material nachgeflossen ist. Anders sind die gut homogenisierten Tone bis in 2 m Bodentiefe nicht zu erklären.

Zur Erklärung für diesen Materialschwund soll die folgende Beobachtung dienen: In der Nähe des Versuchsgeländes befindet sich in Richtung Norden ein Wald, in dem sich zahlreiche Erdfälle befinden, die Dolinen der Gipslaugung aus dem Röt 1 darstellen. Die Versuchsfläche befindet sich in der gleichen Höhe über dem Röt 1, deshalb wird davon ausgegangen, dass auch hier im Untergrund Gipslaugung vorkommt. Da sich das Versuchsgelände an einem leicht nach Süden exponiertem Hang befindet, hat sich der Boden im Periglazialklima stärker erwärmt, als im nördlich gelegenen Gebiet des heutigen Waldes, der nach Norden exponiert ist. Die unterschiedliche Erwärmung beruht also nur auf der Exposition. Deshalb ist von einer höheren Solifluktionsaktivität auszugehen, die dann am Versuchsstandort in Form von Soliplanation stattgefunden hat. Dabei werden die kleineren Unebenheiten nahezu komplett ausgeglichen. Daher kann von der Geomorphologie nicht auf das Liegende geschlossen werden. Dieses erschwert die Erkundung der hydrologischen Verhältnisse an periglazial beeinflussten Pelitstandorten erheblich.

\subsubsection{Tiefenverteilung von Pseudosandanteilen und freiem Carbonat}

Es ist davon auszugehen, dass die Bereiche mit Sandgehalten $>20 \%$-Gew. Bahnen zeigen, in denen, neben den bisher betrachteten Makroporen, bevorzugt Sickerwasser fließt. Zur Visualisierung dieser Bahnen werden die Bereiche $>20 \%$-Gew. markiert (Abbildung 15)

Das Einfallen dieser Lagen nach Norden, sowie in Richtung der Plattenlysimeteranlage wird auch an einer Solifluktionslage verfolgt. Aus Abbildung $15 \mathrm{geht}$ so hervor, dass das Wasser in Richtung der Plattenlysimeteranlage in diesen Lage fließen kann. Die oberste Lage mit Sandgehalten > $20 \%-G e w$. ist nach der Bodenfarbe diejenige, die im Chlorid-Tracerversuchs in Kapitel 4.2.3 die hohen Chloridkonzentrationen aufweist. 


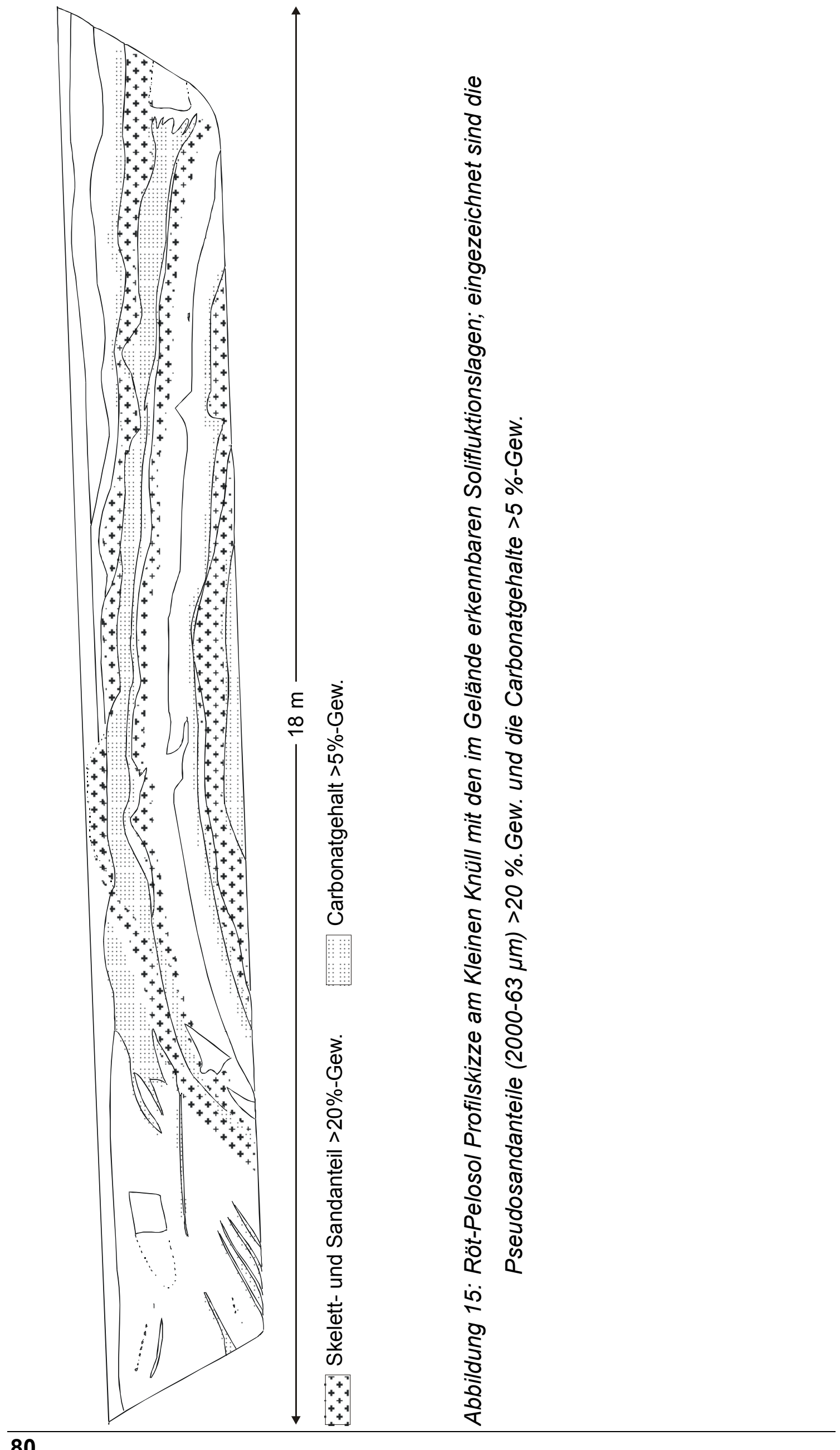


Von den Bereichen mit Carbonatgehalten von > 5 \%-Gew. wird vermutet, dass dort kaum Wasserbewegungen stattfinden, da das primär vorhandene Carbonat nicht durch das Sickerwasser ausgetragen ist. Dies kann in Abbildung 16 aus einem Vertikalprofil in der Mitte des Bodenprofils etwa auf Höhe des Eiskeils erkannt werden: Die Lagen mit hohem Sandanteil sind immer mit niedrigen Carbonatgehalten verbunden. Dies sind neben dem Kluftporensystem des PHorizontes die Zonen des bevorzugten Wasserflusses.

Es schließt sich hier die Frage an, welches der beiden Porensysteme den Hauptbeitrag zur Hydrologie im Boden leistet. Dafür werden im folgenden die pF-Kurven des Akkumulationsprofils und des Verwitterungsprofils betrachtet.

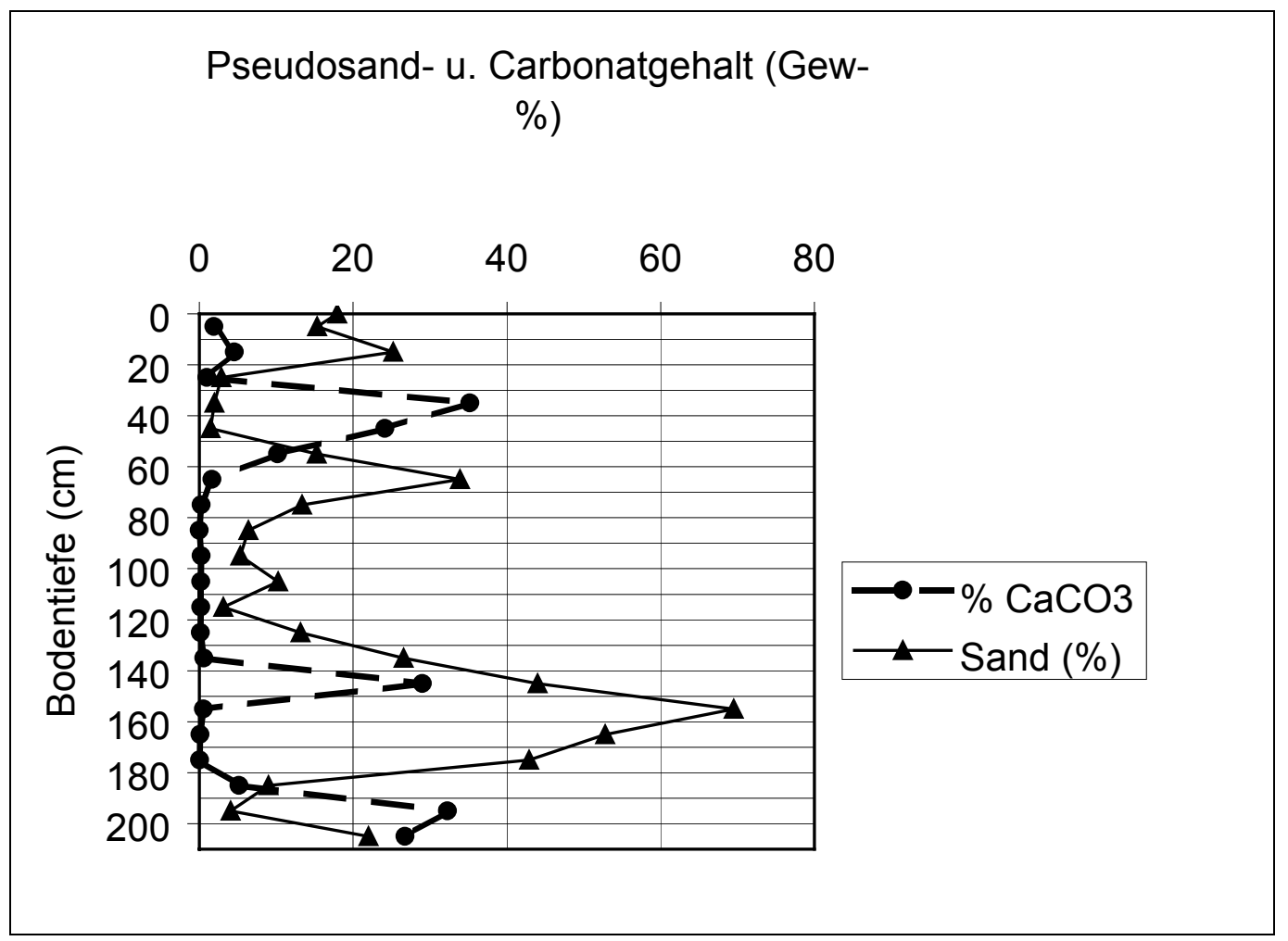

Abbildung 16: Carbonatgehalte (CaCO3) und Summe der Pseudosandanteile (Sand \%-Gew.) in Abhängigkeit von der Bodentiefe in der Mitte des Profilgrabens 


\subsection{Profilansprache}

\subsubsection{Allochthones Pelosol-Akkumulations-Profil}

Tabelle 19: $\quad$ Profilaufbau in der Mitte vom Profilgraben (Allochthones PelosolAkkumulations-Profil)

\begin{tabular}{|l|l|}
\hline Bodentiefe $(\mathrm{cm})$ & Horizontierung und Beschreibung \\
\hline $0-20$ & $\begin{array}{l}\text { Ap (ehemals), leichte Humusanreicherung, Polyedergefüge, } \\
\text { relativ kompakt }\end{array}$ \\
\hline $20-135$ & $\begin{array}{l}\text { P-Horizont, Material gut homogenisiert, untergliedert: oben oh- } \\
\text { ne Manganflecken, Manganflecken in } 60-90 \mathrm{~cm}\end{array}$ \\
\hline $135-160$ & $\begin{array}{l}\text { P-Cv-Übergangshorizont, Mischfarbe aus grauem und rotem } \\
\text { Pelitmaterial, fast komplett homogenisiert, in Polyeder zerfal- } \\
\text { lend }\end{array}$ \\
\hline $160-200$ & Cv, homogener tiefbrauner Horizont, Kompaktgefüge \\
\hline
\end{tabular}

${ }^{*}=$ genaue Angabe der Bodenfarbe nach Munsell-Farbtafel siehe Anhang

Das Profil in Tabelle 19 wird als Typischer Pelosol bezeichnet. Es hat einen sehr mächtigen P-Horizont. Die Einstufung der Reichsbodenschätzung nach $\mathrm{T}$ 5 V 44/39 ist für dieses Profil nachvollziehbar. Man kann den Boden auch wegen der geringen Steinanteile als T 4 V 50/46 einstufen.

\subsubsection{Verwitterungsprofil}

Das Verwitterungsprofil (Tabelle 20) ist wegen der geringen Mächtigkeit des PHorizontes nach der Bodenkundlichen Kartieranleitung (AG BODEN 1994) nicht als Pelosol, sondern als pelosoliger Regosol anzusprechen. Nach Fingerprobe und wegen des hohen Skelettanteiles ergibt die eigene Nachschätzung LT Vg 6 $32 / 27$. 
Tabelle 20: $\quad$ Profilaufbau in der Mitte vom Profilgraben (Verwitterungs-Profil)

\begin{tabular}{|l|l|}
\hline Bodentiefe (cm) & Horizontierung und Beschreibung \\
\hline $0-20$ & $\begin{array}{l}\text { Ap (ehemals), leichte Humusanreicherung, Polyedergefüge, } \\
\text { relativ kompakt }\end{array}$ \\
\hline $20-35$ & $\begin{array}{l}\text { P-Horizont, untergliedert: oben ohne Manganflecken, dann mit } \\
\text { Manganflecken, unten 5-10 cm Vermischung mit der folgenden } \\
\text { Lage }\end{array}$ \\
\hline $35-60$ & $\begin{array}{l}\text { P-Cv-Übergangshorizont, Mischfarbe aus grauem und rotem } \\
\text { Pelitmaterial, steinig u. plattig, z.T. noch in Polyeder zerfallend }\end{array}$ \\
\hline $60-65$ & $\begin{array}{l}\text { Cv, Sandsteinlage (geogen), Sandstein fast aufgelöst teilweise } \\
\text { gebleicht, scharf nach oben und unten abgegrenzt }\end{array}$ \\
\hline $65-90$ & $\begin{array}{l}\text { P-Cv-Übergangshorizont, Kompaktgefüge, im Anschnitt ohne } \\
\text { erkennbare Polyeder, bei Fingerprobe grob, tief rotbraune Far- } \\
\text { be, meist deutlicher Übergang, stellenweise Sandeinlagerun- } \\
\text { gen, in 60-70 cm Tontapeten }\end{array}$ \\
\hline $90-115$ & $\begin{array}{l}\text { Cv, grau, plattig mit roten Pelitplättchen gemischt, Plättchen } \\
\text { waagerecht eingeregelt }\end{array}$ \\
\hline $115-153$ & $\begin{array}{l}\text { Cv, tiefrote Farbe, Kompaktgefüge ohne erkennbare Makropo- } \\
\text { ren }\end{array}$ \\
\hline $153-164$ & $\begin{array}{l}\text { Cv, Sand, z.T. gebleicht } \\
\text { Cv, weniger verwittertes, jedoch mürbes Pelitgestein }\end{array}$ \\
\hline $164-190$ & Cvau kalkig-mergelig, plattig \\
\hline
\end{tabular}

* genaue Angabe der Bodenfarbe nach Munsell-Farbtafel siehe Anhang

\subsection{Bodenphysikalische Parameter}

\subsubsection{Dichteverteilungen}

Pelosole zeigen relativ hohe Trockendichten, die sich meist im Bereich von 1,6 bis $1,8 \mathrm{~g} \mathrm{~cm}^{-3}$ bewegen (Abbildung 17). Es gibt niedrigere Trockendichten bei Profil 1 zwischen 30 und $50 \mathrm{~cm}$ und bei Profil 2 zwischen 40 und $100 \mathrm{~cm}$. Diese Tiefenabschnitte sind bei beiden Profilen durch Beimengungen von grauen, plattigem Material (Gley 5GY 7/1) zu dem rotbraunem Solum (5YR 5/4) gekennzeichnet. Diese Bereiche sind, anders als die Bereiche mit höherer Dichte, nicht so stark miteinander verknetet. Wenn man mit einem Hammer gegen diese Lagen klopft, gibt es ein hohles Geräusch. Makroporen sind dennoch nicht sichtbar. 


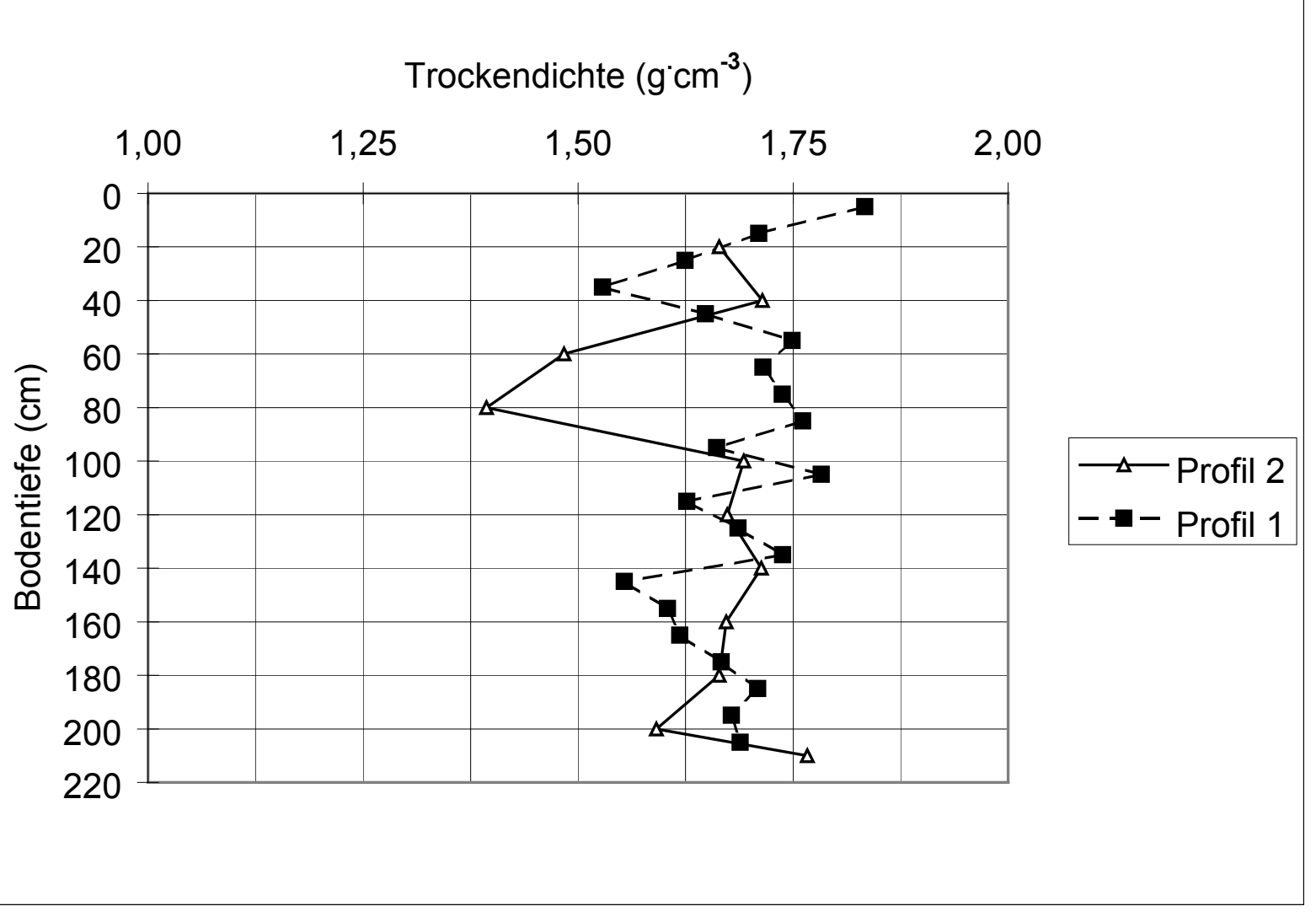

Abbildung 17: Tiefenverteilung der Trockendichte $\left(\mathrm{g} \mathrm{cm}^{-3}\right)$ im VerwitterterungsProfil (Cv) (Profil 1) und im tief verwitterten P-Horizont-Material (Profil 2)

\subsubsection{Wassergehalts-Saugspannungsbeziehung}

Wir erwarten von unseren Pelosolen eine pF-Charakteristik die geprägt ist durch hohe Totwassergehalte $(\mathrm{pF}>4,2)$ um 15-25 \%-Vol. und vergleichsweise niedrige Porenanteile im Bereich pF 2-4,2 bei10-15 \%-Vol. (DIEZ \& WEIGELT, 1991).

Die pF-Charakteristik des Verwitterungsprofiles (Abbildung 18)entspricht nicht diesen für Pelosolen typischen Werten. Sie ähnelt der von GÄTH (1987) für den Ap-Horizont einer Gley-Schwarzerde aus Löss mitgeteilten. 


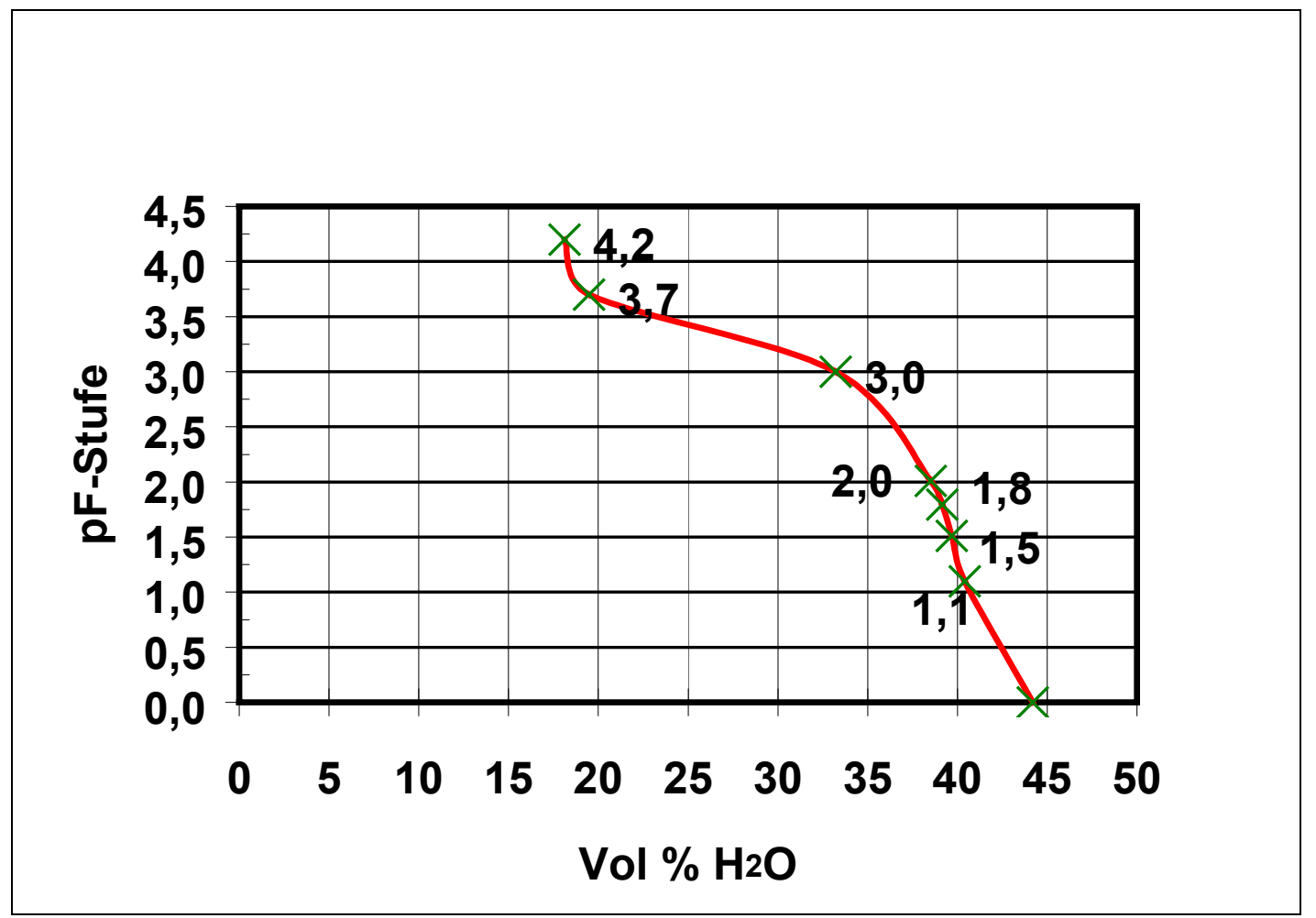

Abbildung 18: Wassergehalts-Saugspannungs-Beziehung von wenig verwitterten Cv-Horizontmaterial (Pelit) aus 60 bis $125 \mathrm{~cm}$ Bodentiefe

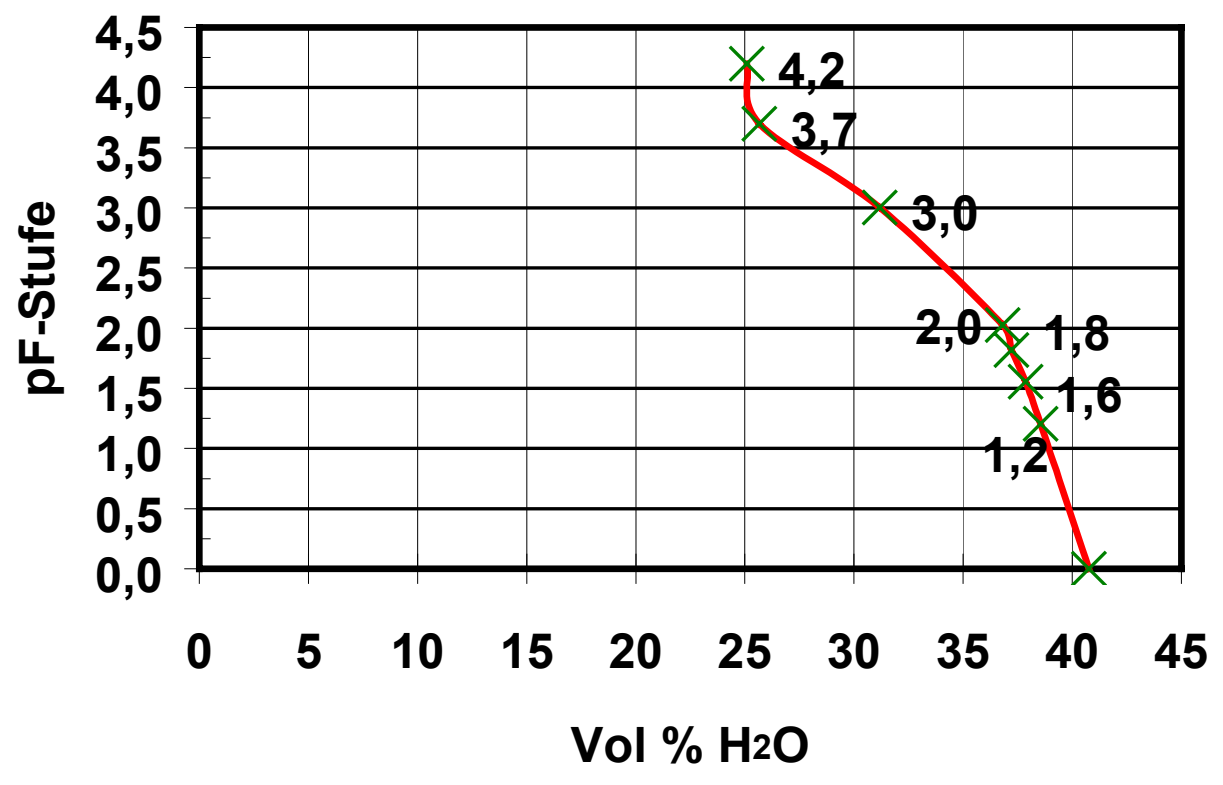

Abbildung 19: Wassergehalts-Saugspannungs-Beziehung vom gut verwitterten P-Horizontmaterial aus 35 bis $200 \mathrm{~cm}$ Bodentiefe 

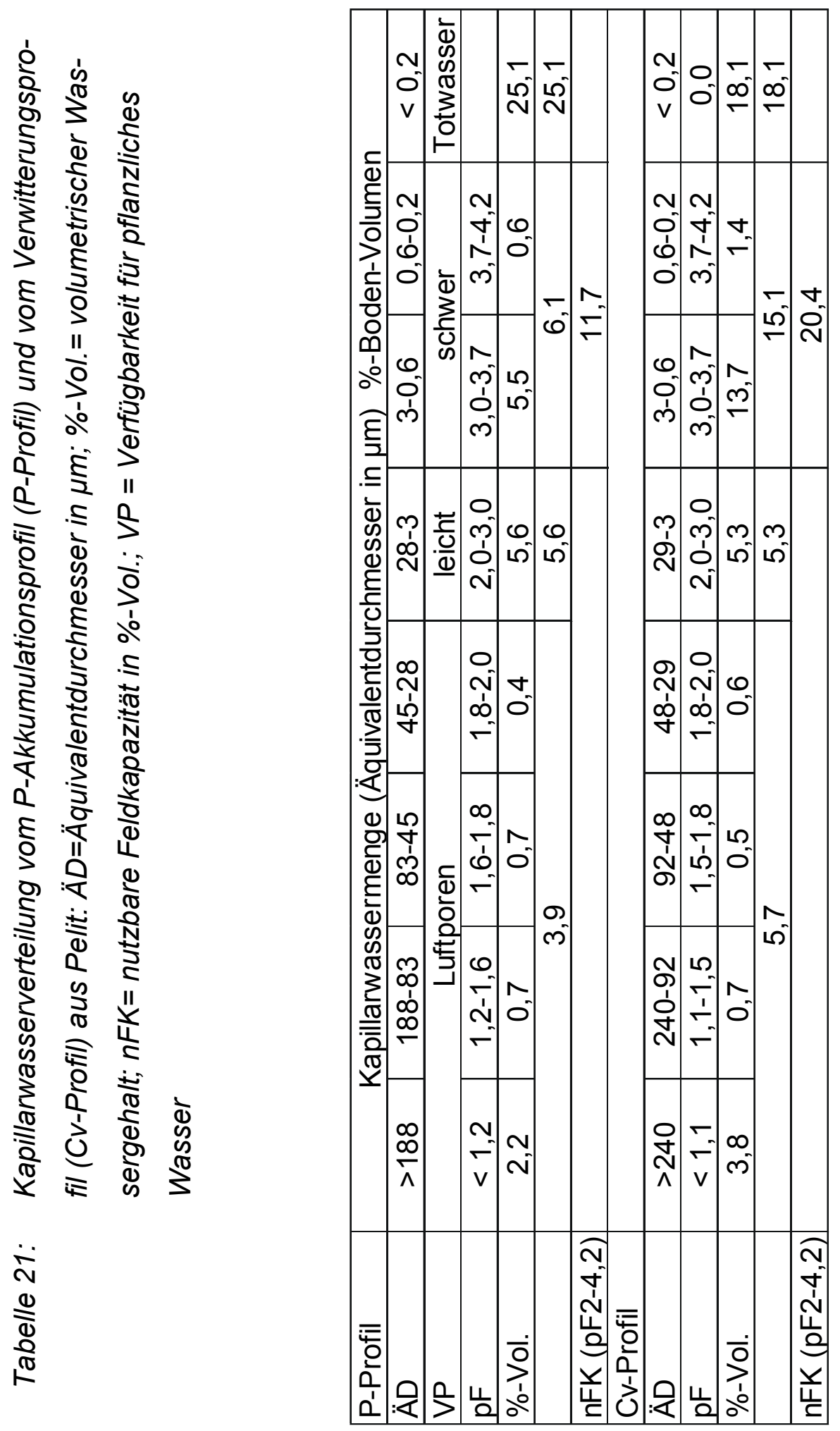

Die pF -Charakteristik des P-Akkumulationshorizontes (Abbildung 19) ist den von KoHL et al. (1971) für typische Pelosole Bayerns sehr ähnlich. 
Die nutzbare Feldkapazität kann aus den vorliegenden Ergebnissen wie in Tabelle 21 kalkuliert werden: In Tabelle 7 sind die Wassergehalte im Cv-Horizont in den Tiefen 26 bis $77 \mathrm{~cm}$ im Frühjahr abgebildet. Dieser hat einen Frühjahrswassergehalt von durchschnittlich 38 \%-Vol. Unter der Annahme, dass die winterlichen Niederschläge die Feldkapazität der Tonböden aufgesättigt haben, geben die 38 \%-Vol. zum Zeitpunkt des Frühjahrs die Feldsättigung wieder. Die pF-Kurve (Abbildung 18) weist diesem Volumenwert einen pF-Wert von 2,0 zu bzw. eine Saugspannung von 100 cm WS. Über die Steighöhengleichung kann man den dabei entwässerten Äquivalentporendurchmesser von 30 um errechnen. Unter Feldbedingungen entwässert der Verwitterungshorizont alle Poren bis $30 \mu \mathrm{m}$ Äquivalentporendurchmesser. Dieses Kalkulationsergebnis ist auch auf das Pelosol-Akkumulationsprofil anwendbar. Die für die Pflanze maximal nutzbare Feldkapazität (nFK) liegt zwischen der Feldkapazität und dem permanenten Welkepunkt (PWP) bei pF 4,2.

$$
\mathrm{nFK}=\mathrm{FK}-\mathrm{PWP} \quad[\%-\mathrm{Vol} .]
$$

Die nutzbare Feldkapazität liegt für das Verwitterungsprofil mit einem permanenten Welkepunkt von 18,1 mit demzufolge bei 20,4 \%-Vol, beim P-

Akkumulationsprofil bei 11,7 \%-Vol. . In Tabelle 21 sind alle relevanten Werte für das pflanzenverfügbare Wasser zusammengestellt. Auffällig ist, dass das pflanzlich verfügbare Wasser sich bei den beiden Profilen nur in der Fraktion des schwer pflanzenverfügbarem Wasser unterscheidet. Dieser Sachverhalt wurde für Lössböden auch von GÄTH (1987) mitgeteilt.

Die Unterschiede der beiden pF-Charakteristiken im Porenbereich von 0,6$3 \mu \mathrm{m}$ Durchmesser (Tabelle 21) können durch rasterelektronische Aufnahmen unterschiedlich stark verwitterter Röt-Materialien von SCHENK (1983) und GRONEMEIER, et al. (1985) folgendermaßen erklärt werden: Die Abbildungen des Mikrogefüges zeigen, dass Proben mit einem geringen Verwitterungsgrad eine sperrige „Kanten-Flächen-Anordnung“, wie bei einem Kartenhaus oder eine „Buchhausstruktur“ haben. Bei dieser Anordnung sind die 0,5 bis mehrere $\mu \mathrm{m}$ große Tonplättchen sehr locker angeordnet sind und visuell sichtbar viele Poren im Bereich 0,6-3 $\mu \mathrm{m}$ Durchmesser vorhanden. Andere Aufnahmen von stärker verwittertem Material von der Bodenoberfläche oder aus solifluidal entstandenen Gleitbereichen zeigen, dass durch die fortschreitende Frostverwitterung die 
Tonplättchen nicht mehr Kante auf Fläche stehen, sondern dass sie zueinander parallel ausgerichtet sind. Die genannten Porenbereiche gibt es nur noch an der Oberfläche der Proben, also in einem sehr viel geringerem Umfang. Der Unterschied zwischen der nutzbaren Feldkapazität der beiden unterschiedlich verwitterten Böden wird insgesamt nur durch die Anordnung des Mikrogefüges verursacht.

Betrachtet man die Tiefenverteilung der Luftporen und Dränporen mit einem Äquivalentdurchmesser von > $30 \mu \mathrm{m}$ in Abbildung 20, so zeigt sich, dass im Pelosol-Akkumulationsprofil die luftleitenden Anteile mit der Bodentiefe relativ stark abfallen. Im Verwitterungs-Profil ist in den gleichen Bodentiefen ein wesentlich höheres Porenvolumen. Die Poren der Matrix in Pelosolen tragen nur unwesentlich zum Wassertransport bei. Im Pelosol-Akkumulationsprofil kann es deshalb zum Wasserstau kommen. Durch die Vernetzung der Vertikalporen entstehen dann laterale Flüsse, wie sie in Kapitel 4.2 dargelegt worden sind.

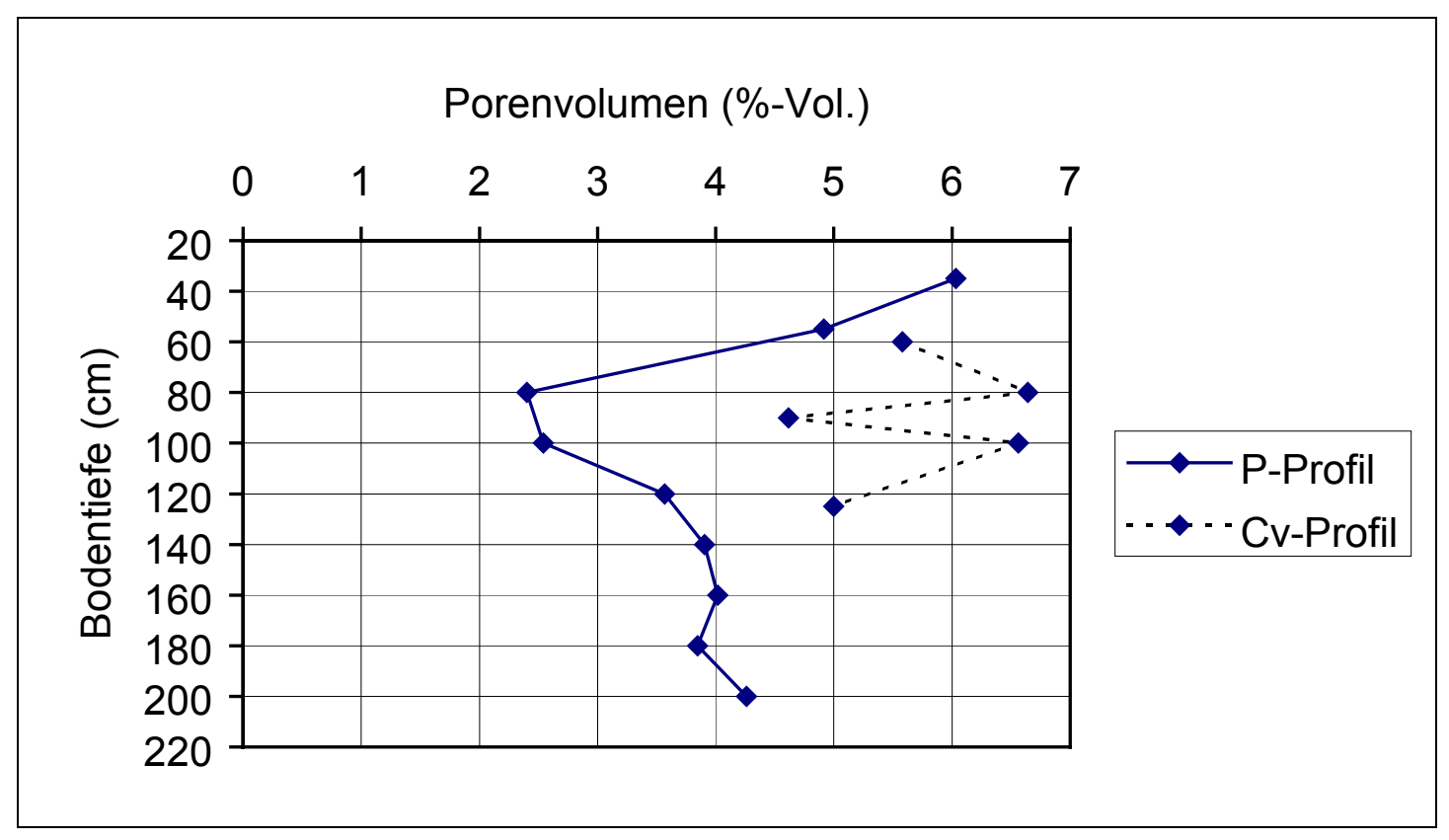

Abbildung 20: Tiefenverlauf der Porenvolumen (\%-Vol.) der Äquivalentporen $>30 \mu \mathrm{m}$ im Pelosol-Akkumulationsprofil (P-Profil) und im Verwitterungsprofil (Cv-Profil)

Außerdem zeigt sich, welche hohe Bedeutung die weniger verwitterten Bereiche für den Wassertransport haben, da hier durchweg zwischen 4,5 und 7 \%- 
Vol. an Poren > $30 \mu \mathrm{m}$ zu finden sind. Dies sind nach BRANDT et al. (1992) die Bereiche, die in Pelosolen die höchsten feldgesättigten Leitfähigkeiten aufweisen.

\subsubsection{Korngrößenverteilungen}

Die Ergebnisse der Methoden zur Bestimmung der Korngrößen unterscheiden sich nicht wesentlich voneinander (Tabelle 22). Dies wurde nicht erwartet, da anhand der pF-Charakteristiken der beiden betrachteten Profile deutlich voneinander unterscheiden.

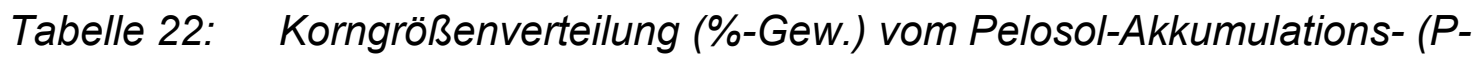
Profil) und Verwitterungsprofil (Cv-Profil). o. Z. = ohne Zerstörung der Kittsubstanzen; m. Z. = mit Zerstörung der Kittsubstanzen; (Erläuterung siehe Methodik)

\begin{tabular}{l|rrr}
\hline O. Z. & Sand & Schluff & Ton \\
P-Profil & 11,24 & 56,77 & 32,57 \\
Cv-Profil & 9,13 & 58,90 & 31,07 \\
\hline & & & \\
\hline m. Z & Sand & Schluff & Ton \\
P-Profil & 10,35 & 58,01 & 32,09 \\
Cv-Profil & 9,19 & 57,66 & 32,30 \\
\hline & & & \\
\hline beide Profile & Sand & Schluff & Ton \\
m. Z. & 10,48 & 57,38 & 32,14 \\
o. Z. & 9,94 & 57,91 & 32,15 \\
\hline
\end{tabular}

Ein Unterschied zwischen den Ergebnissen der beiden unterschiedlich benutzten Korngrößenanalysen wird erst deutlich, wenn die Proben, die Carbonat enthalten, aus der Betrachtung herausgenommen werden (Tabelle 23). Die carbonathaltigen Proben sind durch Dolomit-Kalk miteinander verkittet (GRONEMEIER et al., 1985). Bei der Schlämmanalyse wird das Dolomit im Wasser gelöst. Da dann schon durch das Wasser das Kittmittel gelöst wird, unterscheiden sich die Proben in Tabelle 22 nicht voneinander. 


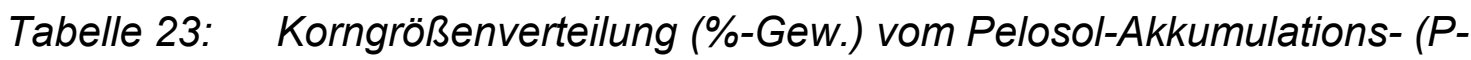
Profil) und Verwitterungsprofil (Cv-Profil) ohne Zerstörung der Kittsubstanzen ohne carbonathaltige Proben; $Q_{T}=$ Quotient aus Totwasservolumen (\%-Vol.) und Tongehalt(\%-Gew.)

\begin{tabular}{lrrrr}
\hline & Sand & Schluff & Ton & $\mathrm{Q}_{\mathrm{T}}$ \\
\hline P-Profil & 12,04 & 56,49 & 31,47 & 0,80 \\
Cv-Profil & 9,42 & 68,51 & 22,08 & 0,82 \\
\hline
\end{tabular}

Bei den roten Proben ( $\sim 5$ YR 4/4) ist Eisen das Kittmittel der Primärteilchen. Für die Schätzung des Totwasseranteils kann nach den Ergebnissen in Tabelle 23 der Tongehalt mit 0,8 multipliziert werden um den Totwasseranteil $(p F>4,2)$ in volumenprozent zu errechnen:

$$
\text { Gew.\% Ton } \cdot 0,8=\text { Vol.\%Totwasser }
$$

Dies haben auch AHL et al. (1996) beschrieben.

\subsection{Schlussfolgerungen}

Die Strukturen der Feinstratigraphie stimmen nicht mit der geomorphologischen Formung der Landschaft überein. Die Prägung durch das Periglazialklima hat einen wesentlichen Einfluss auf die Feinstratigraphie, die wiederum die hydrologischen Parameter in ihrer räumlichen Anordnung maßgeblich beeinflusst. Das Porensystem ist nach der Morphologie weitgehend aus Kluftporen aufgebaut. Es variiert in vertikaler Sicht. Dadurch gibt es laterale Flüsse in Abhängigkeit von der Flussintensität. Die Modellierung eines solche Systems ist nur bei dreidimensionaler Kenntnis der hydrologischen Parameter möglich. Geeignete Messmethoden müssen zur in situ Messung solcher Flüsse entwickelt werden. Anhand der Reichsbodenschätzung wurde gezeigt, dass die Bodenvariabilität im Bereich von mehreren (neun) Metern hoch ist. Für die Bodenkartierung ist es hier besonders schwer, eine repräsentative kleinste Einheit zu finden. Die Bodenschätzung überschätzt das Pelosol-Akkumulationsprofil gegenüber dem Verwitterungs-Profil. Das schlechter geschätzte Verwitterungsprofil hat eine etwa um $10 \%$-Vol. höhere nutzbare Feldkapazität gegenüber dem besser geschätztem Pelosol-Akkumulationsprofil aufzuweisen. Der Unterschied der nutzbaren Feldkapazität kommt durch Unterschiede im Mikrogefüge zustande. 
Makroskopisch für den Bodenschätzer sind die aus kleineren Primärteilchen bestehenden Pseudosande als Sand mit geringer Wasserspeicherung anzusprechen. Diese Pseudosande und Pseudoschluffe leisten jedoch auch einen Beitrag zur Wasserspeicherung des Bodens. Beim Verwitterungsprofil bedingt der hohe Skelettanteil eine weitere Abwertung in der Bodenschätzung. Dieser Sachverhalt sollte in zukünftige Schätzungen aufgenommen werden.

Die Dichteverteilung kann die hydrologischen Parameter des Bodens nicht abbilden. Den beobachteten Pseudogleymerkmalen und den hohen Trockendichten steht ein relativ hohes Luftporenvolumen gegenüber. Dies ist einerseits auf das räumliche Ungleichgewicht der Poren und andererseits auf zeitweiliges Stauwasser zurückzuführen. Die Luftporen und ein Teil der Dränporen (>30 $\mu \mathrm{m}$ ) sind die für den Wassertransport maßgeblichen Poren, bei denen es Bereiche im Profil gibt, in denen die Volumina abnehmen und dadurch Wasser aufgestaut wird.

Die modifizierte Korngrößenanalyse kann zu einem gewissen Grad die hydrologischen Parameter besser abbilden als die nach DIN 19683. 


\section{Schlussbetrachtung}

Die gezeigten Fließstrukturen werden im Wesentlichen durch die bei der Gesteinsgenese entstandenen Schichtung und durch Prozesse des periglazialen Klimas während des Eiszeitalters geprägt. Wichtige Phänomene des Periglazialklimas wie Frostverwitterung, Eiskeile und Solifluktion wurden im Gelände gefunden. Es wurde dargelegt, dass wenig verwitterte Pelite sehr effiziente Wasserleiter sind, deren räumlicher Verlauf wie das Fallen und Streichen solcher Lagen durch Solifluktion und Soliplanation beeinflusst ist und sich nicht an der Geländemorphologie orientieren muss. Deshalb ist ein unterirdischer Zustrom in Trinkwassereinzugsgebieten über die nach der Geomorphologie festgelegten Wasserscheiden als Grenzen hinaus möglich. Durch die geringe Filterwirkung von unverwitterten und wenig verwitterten Peliten ist ein Zustrom von unerwünschten Begleitstoffen aus Bereichen außerhalb von Schutzgebieten in das Trinkwasser möglich. Die Festlegung von Wassereinzugsgebieten muss diese geogenen und periglazial geprägten Strukturen in besonderer Weise berücksichtigen, da die Kluftporen dieser Substrate einen sehr weitreichenden Wassertransport ermöglichen. Besonders erfolgsversprechend erscheint die Kombination von bodenhydrologischen und geohydrologischen Erkundungen, da beide dabei angewendeten Maßstabsebenen für die Gesamteffekte auf den Wasser- und Stofftransport unerlässlich sind.

Die Plattenlysimetermethode zur Erfassung von Wasserhaushaltsgrößen und Transportparametern ist auf solifluidal beeinflussten Pelosolen nicht anwendbar. Zur Erfassung der Wasserhaushaltsgrößen ist die Messung und Abschätzung der Verdunstung durch indirekte, meteorologische Methoden denkbar.

Herkömmliche Stofftransportmodelle, die mit dem „piston flow“ oder dem „miscible displacement"-Ansatz arbeiten, können wegen der präferenziellen Flüsse nicht angewendet werden. Für Parametergewinnung bei der Modellierung der Stoffflüsse müssen geeignete Methoden entwickelt werden, um den hohen räumlichen Variabilitäten und dem dadurch bedingten Wechsel zwischen vertikalen und lateralen Flüssen zu erfassen. Dabei müssen in Zukunft neben den Luftporen (> $300 \mu \mathrm{m}$ ) auch die Dränporen (300-30 $\mu \mathrm{m}$ ) berücksichtigt werden.

Dünnschliffaufnahmen von ALTEMÜLLER (1960 u. 1962) zeigen an Pelosolen des Keuper und des Fränkischen Jura, dass in weniger verwitterten Horizonten von 
Pelosolen gegenüber den stärker verwitterten höhere Porenanteile in den Luftporen (>300 $\mu \mathrm{m})$ und bei den Dränporen (300-30 $\mu \mathrm{m})$ zu finden waren. Die Laborlysimeterversuche zeigen demgegenüber eine Abnahme der gesättigten Leitfähigkeit bei den Bodenmonolithen mit der weniger verwitterten Solifluktionslage gegenüber denen, die nur stärker verwittertes Material enthalten. Betrachtet man jedoch die an Chlorid austauschbaren Porenvolumen, so bestätigt sich, dass die weniger verwitterten Lagen ein höheres Porenvolumen aufweisen. So kann auch das gezeigte laterale Fließen beim Chlorid-Tacerversuch im Felde in weniger verwitterten Solifluktionsbahnen erklärt werden.

Die Bodenschätzung muss für die weniger bis mäßig verwitterten Pelosole modifiziert werden, da die bei einer Feldansprache bestimmten Stein- und Sandanteile aus kleineren Primärteilchen bestehen, die aufgrund ihres Mikrogefüges eine ähnlich hohe nutzbare Feldkapazität wie lössbürtige Böden haben. Im Vergleich dazu werden mächtige Pelosole mit niedrigeren nutzbaren Feldkapazitäten zu hoch geschätzt.

Die Quellung und Schrumpfung hat keine erkennbare hemmende Wirkung auf den Transport von Soluten und suspendierten Partikeln. Wegen der geringen Wasseraufnahme der Matrix im feuchten Zustand ist die potentielle Austragsgefahr im Frühjahr am höchsten. Die Ausbringung von Düngern, Pflanzenschutzmitteln und tierischen Exkrementen sollte deshalb möglichst weit in die Vegetationsperiode hineinverlegt werden, um eine Auswaschung zu vermeiden.

Die durchgeführten Versuche lassen einen Vergleich der Austragsgefährdung für gelöste und suspendierte Stoffe bei verschiedenen Bodenbearbeitungsmaßnahmen zu. Es handelt sich um eine simulierte Winterpflugfurche und um eine pfluglose Bodenbearbeitung (Grasnarbe/Dauerbrache). Auf der Fläche mit der Winterpflugfurche wurde durch eine zusätzliche Bodenbearbeitungsmaßnahme im Frühjahr eine Auswaschung von Oberbodenmaterial in tiefere Bodenschichten verursacht. Eine reduzierte Bodenbearbeitung trägt zur Vermeidung solcher Stoffausträge bei. Ebenso ist zu folgern, dass physiologisch versauernd wirkende Dünger den pH-Wert an den Aggregatoberflächen absenken und dadurch den Ton dispergieren können. Das kann zur zusätzlichen Verlagerung von Oberbodenmaterial führen. 
Die Retentionsleistung für Solute ist differenziert zu betrachten: Bei reduzierter Bodenbearbeitung ist sie, wie die Dauer-Tracerversuche in Kapitel 5 gezeigt haben, äußerst gering. Durch die winterlichen Fröste werden die großen PHorizont-Aggregate in kleine Polyederaggregate zerlegt. Wegen ihrer großen spezifischen Oberfläche und zusätzlichem Porenvolumen durch Eislamellenbildung im Feld-Tracerversuch (Kapitel 4.2) können sie große Mengen an Solut zurückhalten. Durch die Aggregatzerkleinerung ist eine Homogenisierung eingetreten, die dazu führt, dass in der Krume „piston-flow“-Charakter herrscht. Für die sommerliche pflanzliche Nährstoffaufnahme ist die größere spezifische Oberfläche der Aggregate von Vorteil. Nachteilig wirkt sich die Bodenbearbeitung aus, wenn im Herbst größere Mengen mineralischen Stickstoffs aus der Düngung im Boden vorhanden sind. Diese können dann bei abwärts gerichteten Bodenwasserbewegungen durch die folgenden winterlichen Niederschläge leicht ausgewaschen werden. Bei reduzierter Bodenbearbeitung wird dies durch kurze Kontaktzeiten mit den Aggregaten und geringe spezifische Aggregatoberflächen vermindert.

Das einzige Problem der reduzierten Bodenbearbeitung ist, dass die Aufnahme von Wasser und Soluten durch die geringe spezifische Oberfläche der Bodenaggregate langsam verläuft und deshalb die Austragsgefährdung direkt nach der Ausbringung von grundwassergefährdenden Stoffen sehr hoch ist. Insgesamt kann die reduzierte Bodenbearbeitung zur Minderung des Stoffaustrages beitragen und ist deshalb sicherlich unter dem Aspekt des Umweltschutzes gegenüber der intensiven Bodenbearbeitung vorzuziehen. 


\section{Zusammenfassung}

Die Funktion von Peliten und ihren Böden für den Wasser- und Nährstoffhaushalt im Mitteldeutschen Oberen Buntsandstein (Röt) ist nicht geklärt. Der Verbleib des über die Verdunstung hinaus fallenden Niederschlagswassers ist unklar. Einerseits wird bei Untersuchungen zum Landschaftswasserhaushalt davon ausgegangen, dass Tonsubstrate eine hydrologische Barriere bilden, da die gesättigte Wasserleitfähigkeit der Matrix durch die Textur sehr gering ist. Das über die Verdunstung hinaus fallende Niederschlagswasser müsste demnach oberflächlich abfließen. Andererseits steht dazu im Widerspruch die Beobachtung, dass es auf solchen Standorten keine nennenswerten Anzeichen für Oberflächenerosion gibt.

Die Ziele dieser Arbeit waren, die Wasserhaushaltskomponenten sowie die Parameter für den Wasser- und Stofftransport im Jahresgang zu messen. Da man bisher davon ausgegangen ist, dass im gequollenem Zustand kein nennenswerter Wasser- und Stofftransport möglich ist, soll die Frage beantwortet werden, ob eine Tiefeninfiltration vorhanden ist, und ob diese sich unter dem Einfluss des Quellungszustandes des Bodens verändert.

Zur Untersuchung des Wasser- und Stofftransportes wurden Plattenlysimeter und Laborlysimeter eingesetzt. Die für die Hydrologie dieser Böden relevanten Strukturen wurden im Feld an einem $18 \mathrm{~m}$ breiten und $2 \mathrm{~m}$ tiefen Röt-

Pelosolprofil erkundet, welche durch Messung einiger bodenphysikalischer Parameter ergänzt wurden.

Die Ergebnisse der Untersuchungen zur Wasserbewegung und zum Stofftransport sind:

1. Pelosole sind auch im gequollenen Zustand durchlässig. Das steht im Einklang mit der Beobachtung, dass solche Böden selten Oberflächenwasser liefern und keine Quellen enthalten.

2. Lateraler Wassertransport ist in Pelosolen typisch und verbietet die Anwendung von Plattenlysimetern. Die Anteile an lateralen und vertikalen Flüssen variiert in Abhängigkeit von der Niederschlagsintensität. Die lateralen Flüsse orientieren sich nicht an der Morphologie des Geländes, sondern fließen im betrachteten Versuchsfeld entgegen der Hangrichtung. 
3. Wasser- und Stofftransport erfolgt präferenziell in wenigen bevorzugten Bahnen mit hohen Porenwassergeschwindigkeiten.

4. Die an großen Bodenmonolithen gemessene gesättigte Wasserleitfähigkeit ist um zwei Zehnerpotenzen größer als die an Stechzylindern bestimmte.

5. Bei Tracer-Perkolationsversuchen unter gesättigten Bedingungen erfolgt der erste messbare Solutdurchbruch nach 0,01-0,03 durchflossenen Porenvolumen, die Relativkonzentration von 0,5 wird bereits nach 0,2-0,3 Porenvolumen erreicht. Bei einer Durchflussrate von $15 \mathrm{~mm}^{\cdot \mathrm{d}^{-1}} \mathrm{im}$ Dauertracerversuch wird ein ausgeprägtes „tailing“ beobachtet. Die Diffusion aus bevorzugten Bahnen in Bodenaggregate hinein ist sehr gering. Die größte Auswaschungsgefahr im Jahresverlauf ist im zeitigen Frühjahr gegeben.

6. Wassergebundener Partikeltransport wurde durch die Auswaschung von Tonpartikeln aus dem Oberboden nachgewiesen. Diese Partikel können über Dränagen oder den Zwischenabfluss in die Oberflächengewässer gelangen und dort durch das an den Partikeln sorbierte Phosphat zur Euthrophierung beitragen. Die Vergleiche zwischen den Teilversuchen zeigen, dass eine Minderung solcher Austräge durch geeignete Bewirtschaftungsmaßnahmen möglich ist.

7. Das gezeigte laterale Fließen wird geprägt durch die Gesteinsschichtung und durch die Strukturen, die durch das Periglazialklima im Zusammenhang mit dem Eiszeitalter entstanden sind. Frostverwitterung und laminare Solifluktion sind die beiden Prozesse, durch die im Gelände Bereiche herausgeformt werden, die sich im Verwitterungszustand unterscheiden.

8. Die unterschiedliche Verwitterung von Pelitgestein ist nach eigenen Messungen für die bodenphysikalischen Eigenschaften von herausragender Bedeutung. Der Vergleich eines tiefgründigem aufakkumuliertem Pelosolprofils mit einem weniger stark verwitterten Profil zeigt, dass das weniger verwitterte Profil ähnlich wie Löss eine hohe nutzbare Feldkapazität von 20,4 \%Vol. hat, während das Pelosolprofil nur 11,7 \%-Vol. aufweist.

9. Die Betrachtung des landwirtschaftlichen Nutzwertes dieser zwei Profile ergibt, dass die Reichsbodenschätzung dem Profil mit der geringeren nutzbaren Feldkapazität Unzutreffenderweise einen höheren, also besseren Schätzwert zuordnet. Dies wird durch das Mikrogefüge erklärt, welches in die Reichsbodenschätzung nicht eingeht. 


\section{Literatur}

AG BODEN (1994): Bodenkundliche Kartieranleitung. 4. verb. u. erw. Aufl., Hannover, $392 \mathrm{~S}$.

Aht, C.; Becker, K.- W.; Jörgensen, R. G.; Klages, F.- W. \& Wildhagen, H. (1996): Aspekte und Grundlagen der Bodenkunde. -Vorlesungsskript des Inst. für Bodenwissenschaften Göttingen, Selbstverlag, 1-304

AK BODENSYSTEMATIK (1998): Systamatik der Böden und der bodenbildenen

Substrate Deutschlands. Mitt. Dt. Bodenkundl. Ges. 86: 1-180

AltemÜLleR, H.- J. (1960): Mirkromorphologische Untersuchungen an einigen Gipskeuperböden im Raum Iphofen. Bayer. Landw. Jb. 구, Sonderheft 4: $70-85$

AltemÜLleR, H.- J. (1962): Mirkromorphologische Untersuchungen an Bodenprofilen des mittleren fränkischen Juraeinigen. Bayer. Landw. Jb. $\underline{39}$, Sonderheft 1: 98-109

BACH, M. (1987) Die potentielle Nitratbelastung des Sickerwassers durch die Landwirtschaft in der Bundesrepublik Deutschland. Göttinger Bodenkundl. Ber. 93: 1-186

Barry, D. A., Parlange, J.- Y., Haverkamp, R. \& Ross, P. J. (1995): Infitration under ponded conditions: 4. An explicit predictive infiltration formula. Soil Sci. 160 (1): 8-17

BECHER, H. H. (1985): Mögliche Auswirkungen einer schnellen Wasserbewegung in Böden mit Makroporen auf den Stofftransport. Z. dt. geol. Ges. 136: 303-309.

Beese, F. \& VAn DeR Ploeg, R. R. (1979): Simulation des Anionen-Transportes in ungestörten Bodensäulen unter stationären Fließbedingungen. $\mathbf{Z}$. Pflanzenernähr. Bodenkd. 142: 69-85

BEISECKER, R. (1993): Einfluß langjährig unterschiedlicher Bodenbearbeitungssysteme auf das Bodengefüge, die Wasserinfiltration und die Stoffverlagerung eines Löß- und eines Sandbodens. Bodenökologie und Bodengenese: 12: 1-182

BeVEn, K. J. \& Young, P. C. (1988): An agregated mixing zone model of solute transport through porous media. J. Contam. Hydrol. ㅁ: 129-143

BiggaR, J. W. \& Nielsen, D. R. (1962): Miscible displacement in soils: II. Behavior of tracers. Soil Sci. Soc. Am. Proc. 26: 125-128 
BITTON, G. \& HARVEY, R. W. (1992): Transport of pathogens through soils and aquifers. Environm. Microbiol. ㅁ: 103-124

Blake, G., SChlichting, E. \& ZimmermanN, U. (1973): Water recharge in a soil with shrinkage cracks. Soil Sci. Soc. Am. Proc. 37 (4): 669-672

BLoom, A. L. (1998): Geomorphologie: A systematic analysis of late cenozoic landforms. $3^{\text {rd }}$ Edition. Prentice Hall, New Jersey, 1-482

BÖHM, W. (1979): Methods of studying root systems. - Berlin [u.a.] : Springer, (Ecological studies 33) $188 \mathrm{~S}$.

BOHNE, K. (1996): Möglichkeiten und Grenzen der Simulation des Wasser- und Stofftransportes in mineralischen Substraten mit Hilfe von Modellen. $Z$. Kuturtechn. Landentw. 37: 40-47

Bond, W. J.; GARDiner, B. N. \& Smiles, D. E. (1984): Movement of $\mathrm{CaCl}_{2}$ Solutions in a unsaturated clay soil: The effect of solution concentration.

Austr. J. Soil Res. 22: 43-58

BOOLTINK, H. G. W. (1995): Field monitoring of nitrate leaching and water flow in a structured clay soil. Agriculture Ecosys. Environm. 52: 251-261

BÖtTCher, J.; FREDE, H.- G. \& MeYER, B. (1983): Chlorid- und Nitratverlagerung in Löss-Lysimetern bei unterschiedlichen Grundwasserständen. Mitt. Dt. Bodenk. Ges. 38: 65-70

BoumA, J. \& BOOLTINK, H. G. W. (1990): Using soils porosity analysis to improve characterization of water movement in heavy clay soils. Transactions 14 ${ }^{\text {th }}$. Int. Congr. Soil Sci. Soc. Am., Kyoto, Japan Vol.IV: 146-151

BOUMA, J. \& DEKKER, L. W. (1978): A case study on infiltration into dry clay soil. I. Morphological observations. Geoderma 20, 27-40

BOUMA, J. \& WÖSTEN, J. H. M. (1984): Characterizing ponded infiltration in a dry cracked clay soil. J. Hydrol. 69: 297-304

BoumA, J. (1984): Using soil morphology to develop measurement methods and simulation techniques for water movement in heavy clay soils. Proc. ISSS Symposium on Water and Solute Movement in Heavy Clay. ILRI Pub. 37: 298-315

BoumA, J., DekKer, L. W. \& Wösten, J. H. M. (1978): A case study on infiltration into dry clay soil. II. Physical measurements. Geoderma 20, 41-51

BoumA, J.; DeKKER, L. W. \& MUILWIJK, C. J. (1981): A field method for measuring short-circuiting in clay soils. J. Hydrol. $\underline{52}$ : 347-354 
BRANDT, M. (1993): Pelite und ihre Böden: Landschaftshydrologische Funktion in Abhängigkeit von geo- und pedogener Struktur und saisonalen Schwankungen. Unveröffentl. Dipl.-Arbeit Inst. f. Bodenwissensch. Univ. Göttingen, $82 \mathrm{~S}$.

Brandt, M.; Hasselbauer, R. \& Meyer, B. (1992): Pelite und ihre Böden: Landschaftshydrologische Funktion in Abhängigkeit von Geo- und pedogener Strukturierung und saisonalen Schwankungen:-Beispiel WEG Hameln-Süd-. Mitt. Dt. Bodenk. Ges. 68: 41-44

BrInKMANN, R (1991): Abriß der Geologie. Bd.2: Historische Geologie. -Enke Verlag Stuttgart. 1-404

BRusseAU, M. L. \& RAO, P. S. C. (1990): Modelling solute transport in structured soils: A review. Geoderma 46: 169-192

BÜDEL, J (1937): Eiszeitliche in rezente Verwitterung und Abtragung im ehemals nicht vereisten Teil Mitteleuropas. Petermanns Geographische Mitteilungen, Ergänzungsheft 229 (Perthes Verlag Gotha): 1-71

DANZER, P. (1964): Untersuchungen zur Stratigraphie und Fazies der Röt-Folge im Oberen Buntsandstein der Rhön, Diss. Univ. Frankfurt a. M., 69 S.

DE MOL, F. (1996): Bodenwasserbewegung und Bodenstruktur im Makroporenbereich, Dissertation FB Agrarw. Univ. Göttingen, 116 S.

De Smedt, F.; Wierenga P. J. \& VAN DER Reken, A. (1981): Theoretical and experimental study of solute movement through porous media with mobile and immobile water. Diss. Vrije Univ Brüssel, 219 S.

DEKKER, L. W. \& RITSEMA, C. J. (1994): How water moves in a water repellent sandy soil: I. Potential and actual water repellency. Water Resour. Res. 30 (9): 2507-2517

DEKKER, L. W. \& RITSEMA, C. J. (1996): Preferential flow paths in a water repellent clay soil with grass cover. Water Resour. Res. 32 (5): 1239-1249

DIEZ, T \& WEIGELT, H. (1987): Böden unter landwirtschaftlicher Nutzung. -Verlagsunion Agrar, BLV Verlagsgesellschaft, $123 \mathrm{~S}$.

DIEZ, T. (1959): Entwicklung und Eigenschaften von Böden aus tonigen Substraten, Diss. Rhein.-Wilh.-Univ. Bonn, $145 \mathrm{~S}$.

DöLL, P (1996): Modeling of moisture movement under the influence of temperature gradients: Desiccation of mineral liners below landfills. Diss., TU Berlin, $251 \mathrm{~S}$. 
Droogers, P.; Stein, A.; BoumA, J. \& De Boer, G. (1997): Parameters for describing soil macroporosity derived from staining patterns. Geoderma $\underline{\mathbf{8 3}}$ : 293-308

DVWK (1980): Empfehlungen zum Bau und Betrieb von Lysimetern, DVWKRegeln zur Wasserwirtschaft, H. 114, Kommissionsverlag Paul Parey, Hamburg und Berlin, $52 \mathrm{~S}$.

FLÖRKEMEYER, H. (1990): Regeneration der Primärstruktur verdichteter Lössböden-Körper durch Frost. Diss. FB Agrarw. Univ. Göttingen, 143 S.

Food ANd Agriculture Organization of the United Nations (1990): Soil map of the world. Revised Legend. World soil resources reports $\underline{60}: 1-119$

FRIEDRICH, W. \& FRANZEN, H. (1960): Ein neuer Versickerungsmesser. Dt. gewässerkundI. Mitt. 4 (1): 105-111

GAESE, D. (1979): Gefügedynamik und Wasserbewegung in Pelosolen. Diss. Univ. Hohenheim, 196 S.

GARDNER, W. R. (1956): Calculation of capillary conductivity from pressure plate outflow data. Soil Sci. Soc. Am. Proc. 20: 317-320

GÄTH, S. (1987): Die Böden aus Löss in ihrer hydrologisch differenzierten Gesellschaft in Börde- und Hügellandschaften Südniedersachsens. Diss. Univ. Göttingen, $247 \mathrm{~S}$.

GÄTH, S., LÜTKEMÖlleR, D., Nöhles, I. \& FREDE, H.- G. (1996): Transportverhalten von Bromid in Löß-Säulen mit und ohne Makropore. Z Kulturtechn. Landentw. 1: 19-23

Geries, H. (1991): Nitrat unter Acker in mächtigen Lössdecken mit tiefem Grundwasserstand im Klimaraum Südniedersachsen: TiefenVerlagerung, Datierung, Umsatz und Einbindung in den Gashaushalt. Diss. Univ. Göttingen, FB. Agrarwiss. $146 \mathrm{~S}$.

GeRKE, H.- H. \& VAN Genuchten, M. T. (1993): A dual porosity model for simulating the preferential movement of water and solutes in structured porous media. Water Resour. Res., 29 (2): 305-319

GermanN, P. \& BÜRgI, T. (1996): Kinematischer Ansatz zur in-situ-Erfassung des Makroporenflusses in Böden während Infiltrationen. Z. Kulturtechn. Landentw. 37: 221-226

GermanN, P. F., Smith, M. S. \& ThomAS, G. W. (1987): Kinematic wave approximation to the transport of Escherichia coli in the vadose zone. Water 
Resour. Res. 23 (7): 1281-1287

Groenvelt, P. H. \& Bolt, G. H. (1972): Water Retention in soil. Soil Sci. 113 (4): $238-245$

Haria, A. H., Johnson, A. C., Bell, J. P. \& BatcheloR, C. H. (1994): Water movement and isoproturon behavior in a drained heavy clay soil: 1 . Preferential flow processes. J. Hydrol. 163: 203-216

HARTGE, K. H. \& HORN, R. (1989): Die Physikalische Untersuchung von Böden. 2., völlig neu bearbeitete Aufl., Enke-Verlag Stuttgart, $175 \mathrm{~S}$.

HARTGE, K. H. \& HORN, R. (1991): Einführung in die Bodenphysik. 2. überarb. u. erw. Aufl. Enke-Verlag Stuttgart, $303 \mathrm{~S}$.

HASE, D \& MEYER, B (1969): Feuchte-Fahresgang, Wasser-Bewegungen und Bilanzen in dicken Würmlöß-Decken und ihren holozänen Böden (Parabraunerde, Griserde, Feuchtschwarzerde) bei unterschiedlichem Grundwasserstand im Raum Niedersachsen. Götting. Bodenkundl. Ber. 11: 85-183

HATANO, R. \& BOOLTINK, H. W. G. (1992): Using fractal dimensions of stained flow patterns in a clay soil to predict bypass flow. J. Hydrol. 135: 121131

HEYDER, D (1993): Nitratverlagerung, Wasserhaushalt und Denitrifikationspotential in mächtigen Lössdecken und einem Tonboden bei unterschiedlicher Bewirtschaftung. Bonner Bodenkundliche Abhandlungen 10: $1-171$

HINZE, C. (1967): Der Obere Buntsandstein (Röt) im südniedersächsischen Bergland. Geol. Jb. 84: 637-716.

HöLTING, B. (1992): Hydrogeologie : Einführung in die allgemeine und angewandte Hydrogeologie. 4. überarb. Aufl. - Stuttgart: Enke-Verlag, $415 \mathrm{~S}$. Hopmans, J. W; Vogel, T \& KobliK, P. D. (1992): X-ray Tomography of soil water distribution in one-step outflow experiments. Soil Sci. Soc. Am. J. 45: 355-362

JoERgENSEN, R. G. \& SeITZ, D. (1998): Depth transfer of fecal organisms in a arable clayley soil. Z. Pflanzenernähr. Bodenk. 161 (5): 309-314

JÖRGENSEN, R. G. (1987): Flüsse, Umsatz und Haushalt der postmortalen organischen Substanz und ihrer Stoffgruppen in Streudecke und Bodenkörper eines Buchenwald-Ökosystems auf Kalkgestein. Göttinger boden- 
kundl. Ber. 91: 1-409

KENKEL, A. (1997): Wasser- und Stoffhaushalt im landwirtschaftlich genutzten Trinkwassereinzugsgebiet Gelliehausen (Gemeinde Gleichen). Dissertation Math.-Nat. Fak. Univ. Göttingen, 222 S.

KISSEL, D. E.; RitCHIE, J. T. \& BURNETT, E. (1973): Chloride movement in undisturbed swelling clay soil. Soil Sci. Soc. Am. Proc. 37: 21-24

KOHL,F.; DiEZ, T.; JERZ, H. \& WiTtMANN, O. (1971): Bodenlandschaften und Böden in Bayern. Mitt. Dt. Bodenk. Ges. 13: 479-521

KRAKOW, L. (1990): Mesozoische Tongesteine in Südniedersachsen und ihre Verwendbarkeit als Dichtungsmaterial für Deponien. Dissertation Math.Nat. Fak. Univ. Göttingen, $440 \mathrm{~S}$.

KutíleK, M. \& NiELSEN, D. R. (1994): Soil hydrology: Textbook for students of soil science, agriculture, forestry, geoecology, hydrology, geomorphology or other related disciplines. - Cremlingen-Destedt: Catena-Verl., 370 S.

LiedTKE, H. (Hrsg.) (1995): Physische Geographie Deutschlands. - 2., durchges. Aufl. - Gotha : Perthes-Verlag, 559 S.

LogSDON, S. D. (1995): Flow mechanisms through continuous and buried macropores. Soil Sci. 160 (4): 237-242.

MACKIE, L. A.; MullinS, C. E. \& FRITZPATRICK, E. A. (1984): Structural changes in two clay soils under contrasting systems of management. . Proc. ISSS Symposium on Water and Solute Movement in Heavy Clay. ILRI Pub. 37: 71-77

McKenzie, N. J.; Jaquier, D. J. \& Ringrose-VoAse, A. J. (1994): A rapid method for estimating soil shrinkage. Austr. J. Soil Res. 32 (5): 931-938

MCLAUGHLIN, M. J. (1982): A review of the use of dyes as soil water tracers. Water SA 8 (4): 196-201

MEIWES, K.- J. (1974): Die Bodengesellschaft der Rötton-BausandsteinLandschaft im Raum Göttingen - Kartographische Aufnahme und Analyse einer landschaftstypischen Bodencatena. Unveröffentl. Dipl.-Arbeit Landw. Fak. Univ. Göttingen, 97 S.

MESSING, I. \& JARVIS, N. J. (1990): Seasonal variation in field-saturated hydraulic conductivity in two swelling clay soils in Schweden. J. Soil Sci. 41: 229-237 
MESSING, I. \& JARVIS, N. J. (1995). A comparison of near-saturated hydraulic properties measured in small cores and large monoliths in a clay soil. Soil Techn., $\underline{7}$ (4): 291-302

MEYER, B. (1999): mündliche Mitteilung MüCKENHAUSEN, E. (1985): Die Bodenkunde und ihre geologischen, geomorphologischen, mineralogischen und petrologischen Grundlagen. 4. erg. Aufl., DLG-Verlag, Frankfurt a. M., 579 S.

MuNCH, J. C. \& DoMINIK, H. P. (1991): Verhalten und Versickerung von fäkalen Organismen nach Gülleausbringung auf typische Böden des Langenauer Donaurieds. VDLUFA Kongreßband: 613-618

Munsell Soll Color ChARTS (1975): Munsell Color Company, Inc. Baltimore NASSAR, I. N., BENJAMIN, J. G. \& HORTON, R. (1996): Thermally induced water movement in uniform clayey soil. Soil Science 161: 471-479

Nielsen, D. R. \& BiggaR, J. W. (1961): Miscible displacement in soils: I. Experimental information. Soil Sci. Soc. Proc. 25 (1) : 1-5

OlBERTZ, M. H. (1957): Über die am Standort des Kulturbodens erfaßbaren Größen des Wasserhaushaltes. Wiss. Abh. Dt. Akad. Landw. Wiss. Berlin 23: 1-109

OOstindie, K. \& BRonswiJK, J. J. B. (1995): Consequences of preferential flow in cracking clay soils for contamination-risk of shallow aquifers. J. Environm. Managem. 43: 359-373

Plagge, R. (1991): Bestimmung der ungesättigten Leitfähigkeit im Boden. Bodenökologie und Bodengenese: ㄱ: 1-152

PÖRTGE, K.- H. (1979): Oberflächenabfluß und aquatischer Materialtransport in zwei kleinen Einzugsgebieten östlich Göttingen (Südniedersachsen), Dissertation Math.-Nat. Fak. Göttingen, 125 S.

PÖRTGE, K.- H. (1996): Tagesperiodische Schwankungen des Abflusses in kleinen Einzugsgebieten als Ausdruck komplexer Wasser- und Stoffflüsse. Göttinger Geograph. Abhandl. 104: 1-104

RogowsKI, A. S. (1988): Flux density and breakthrough times for water and tracer in a spatially variable, compacted clay soil. J. Contam. Hydrol. $\underline{\mathbf{3}}$ : 327-348

RoHDENBURG, H. (1971): Einführung in die klimagenetische Geomorphologie anhand eines Systems von Modellvorstellungen am Beispiel des fluvi- 
alen Abtragungsreliefs. -Lenz Verlag Giessen, $350 \mathrm{~S}$.

ROHDENBURG, H. (1989): Landschaftsökologie - Geomorphologie. CremlingenDestedt: Catena-Verlag, $220 \mathrm{~S}$.

Roth, D.; GÜNTHER, R. \& BREITSCHUH, G. (1989): Untersuchungen zum Wasserverbrauch von Winterweizen, Sommergerste, Zuckerrübe und Kartoffeln unter Feldbedingungen auf einem tiefgründigen Lößstandort. Die Bodenkultur $\underline{40}$ (4):305-319

Roth, K. (1996): The role of modeling and simulation in soil physical research.

Z. Kulturtechn. Landentw. 37: 32-39

SaffingnA, P. G.; Keeney, D. R. \& TAnner, C. B. (1977): Lysimeter and field measurements of chloride and bromide leaching in an uncultivated loamy sand. Soil Sci. Soc. Am. J. 41: 478-482

SCHIFF, H. (1971): Meteorologische Lysimeteruntersuchungen (Wasserhaushalt des Bodens, Abhängigkeit von meteorologischen Einflußgrößen und Wetterlagen): Ber. Inst. Meterorol. Klimatol. TU Hannover $\underline{5}$ : 1-131

SCHINDLER, U. (1980): Ein Schnellverfahren zur Messung der Wasserleitfähigkeit im teilgesättigten Boden an Stechzylinderproben. Arch.Acker-, Pflanzenbau Bodenkd. 24: 1-7

SCHIRMER, H. \& VENT-SCHMIDT, V. (1979): Mittlere Niederschläge für Monate und Jahr. - In: Dtsch. Wetterdienst [Hrsg.]: Das Klima der Bundesrepublik Deutschland. Selbstverlag Offenbach, $70 \mathrm{~S}$.

SCHLINDWEIN, S. L. (1992): Dispersiver Tracer- und Austauschionen-Transport bei geringem ungesättigtem Flux durch Aggregat-Packungen des Basalt-Latosolo-Roxo Südbrasiliens. Göttinger Bodenk. Ber. 101: 1-152

SCHRÖDTER, H. (1985): Verdunstung: Anwendungsorientierte Meßverfahren und Bestimmungsmethoden. Springer Berlin, $186 \mathrm{~S}$.

SCHRÖTER, J. (1983): Der Einfluß von Textur- und Struktureigenschaften poröser Medien auf die Dispersivität, Diss. Math- Naturw. Fak. Univ. Kiel, $152 \mathrm{~S}$.

SCHULIN, R.; WiERENGA, P.J. \& FlÜHLER, H. (1984): Solute displacement through a rendzina. Proc. ISSS Symposium on Water and Solute Movement in Heavy Clay. ILRI Pub. 37: 262-267

SCHULTZ, J. (1988): Die Ökozonen der Erde. -Verlag Eugen Ulmer, Stuttgart. $488 \mathrm{~S}$. 
SCHWEIKLE, V. (1982): Gefügeeigenschaften von Tonböden - Quellung und Schrumpfung als gefügeprägende Prozesse in Pelosolen (und Vertisolen): Hohenheimer Arbeiten 117: 1-79

Shackelford, C. D., Daniel, D. E. \& LilJestrand, H. M. (1989): Diffusion of inorganic chemical species in compacted clay soil. J. Contam. Hydrol. 4: $241-273$

SIEBNER, C. (1995): Lysimeterversuche zum Einfluß verschiedener Vegetationsformen auf den Wasser- und Nährstoffhaushalt von Sandböden der Colbitz-Letzlinger Heide. Unveröffentl. Dipl.-Arbeit FB Agrarwissensch. Univ. Göttingen, $87 \mathrm{~S}$.

SMILES, D. E; GARDINER, B. N. 1982: Hydrodynamic dispersion during unsteady, unsaturated water flow in a clay soil. Soil Sci. Soc.Am. J. $\underline{46}$ (1): 9-14

SMITH, D. J. (1992): Long-term rates of contempory solifluction in the canadian rocky mountains. -in: Periclacial Geomorphology. Ed. J.C.Dixon \& A.D. Abrahams. -John Wiley and Sons Ltd., Chichester, England

SpANGENBERG, A. (1997): Heterogenität und Dynamik der Bodenlösungschemie am Beispiel verschiedener Nährelemente im Freiland und TracerTransport an Bodensäulen. Ber. Forschungszentr. Waldökosys. 149: $1-169$

SPONAGEL, H. (1980): Zur Bestimmung der realen Evapotranspiration landwirtschaftlicher Kulturpflanzen. Geolog. Jahrb., Reihe F $\underline{\mathbf{g}}$

Stolte, J.; FreiJer, J. I.; Bouten, W.; DiRksen, C.; Halbertsma, J. M.; VAN Dam, J. C.; van den Berg, J. A.; Veerman, G. J. \& Wösten, J. H. M. (1994): Comparison of six methods to determine unsaturated hydraulic conductivity. Soil Sci. Soc. Am. J. 푸: 1596-1603

Titus, B. D. \& MAHENDRAPPA, M. K. (1996): Lysimeter system designs used in soil research: A review. Canadian Forest Service. Information Report (Canadian Forest Service, New Foundland and Labrador Region), N-X-301, $113 \mathrm{~S}$.

Toorman, A. F.; Wierenga, P. J. \& Hills, R. G. (1992): Parameter estimation of hycdraulic properties from one-step outflow data. Water Resour. Res $\underline{28}$ (11): 3021-3028

TYLER, D. D. \& ThOMAS, G. W. (1981): Chloride movement in undisturbed soil columns. . Soil Sci. Soc. Am. J. 45: 459-461 
VAN DAM, J. C.; StRICKER, J. N. M. \& DROOgeRS, P. (1990): Inverse method to determine soil hydraulic functions from one-step outflow experiments.

Soil Sci. Soc. Am. J. 56: 1042-1050

VAN DAM, J. C.; StRickeR, J. N. M. \& DRoogers, P. (1994): Inverse method to determine soil hydraulic functions from multistep outflow experiments. Soil Sci. Soc. Am. J. 58: 647-652

van der Ploeg, R. R. \& Benecke, P. (1972): Miscible displacement in soils. Mitt. Dt. Bodenkundl. Ges. 16: 179-206

VAN HOYNINGEN-HuENE, J. \& BRAMm, A. (1978): Die wägbare Unterdrucklysimeteranlage in Braunschweig- Völkenrode. Landbauforschung Völkenrode 28: 95- 102

Vereecken, H.; KaISER, R.; Dust, M. \& PüTZ, T. (1990): Evaluation of the multistep outflow method for the determination of unsaturated hydraulic properties of soils. Soil Sci. 162 (9): 618-631

Vereecken, H.; MAES, J. \& FeYEN, J. (1990): Estimating unsaturated hydraulic conductivity from easily measured soil properties. Soil Sci. 149 (1): 112

WAGNER, J.- A. (1993): Untersuchungen zur Tenazität und zum Infiltrationsverhalten von Salmonellen und Güllekeimen in Standardböden und in verschiedenen Böden des Wasserschutzgebietes Donauried, Diss. Univ. Hohenheim, $185 \mathrm{~S}$.

Webb, E. K. \& ANDERson, M. P. (1996):Simulation of preferential flow in threedimensional, heterogeneous conductivity fields with realistic internal architecture. Water Resour. Res. 32 (3): 533-545

WEIGEL, T. (1995): Untersuchungen des Infiltrationsverhaltens von Mikroorganismen in Böden mittels Gruben- und Laborversuchen sowie eines selbst entwickelten Prototyps zur Probennahme ohne Sekundärkontamination, Diss. Univ. Hohenheim, $119 \mathrm{~S}$.

WEINZIERL, W. (1984): Wasserhaushalt und Ertrag landwirtschaftlicher Kulturpflanzen ausgewählter Löss-Standorte im Raum Göttingen. Gött. Bodenkundl. Ber. 81: 1-105

WENDROTH, O (1990): Koeffizienten des Wasser- und Gastransportes zur Ableitung von Kenngrößen des Bodengefüges. Diss. FB Agrarwiss. Univ. Göttingen, 179 S. 
WENZEL, B. (1994): Zur Lithostratigraphie und Sedimentologie des Röt und zu den Massenverlagerungen an der Röt / Muschelkalkgrenze in Nordosthessen. Giessener Geolog. Schr. 53: 1-379

WHITE, R. E. (1985): The influence of macropores on the transport of dissolved and suspended matter through soil. Adv. Soil Sci. ㄱ: 95-120

WILHELMI, H.- G. (1994): Oberirdischer Abfluß, Gebietswasserbilanz und abflusswirksame Wasservorräte in einer Röt-Pelit-Landschaft Südniedersachsens. Ein Beitrag zur Abschätzung der Aquifer-Funktion von PelitLandschaften. Unveröffentl. Diplom-Arbeit FB Agrarwissenschaften, Univ. Göttingen, $75 \mathrm{~S}$.

ZHANG, R. (1995): Prediction of solute transport using a transfer function model and the convection-dispersion equitation. Soil Sci. 160 (1): 18-27

ZURMÜHL, T. \& DURNER, W. (1996): Modeling transient water and solute transport in a biporous soil. Water Resour. Res. 32 (4): 819-829 


\section{Anhang}

Anhang A: Niederschläge $(\mathrm{N})$, Beregnung (Ber.) und Sickerwasser aus tiefengestaffelten Lysimetern $(30-90 \mathrm{~cm})$ in $\mathrm{mm}$

\begin{tabular}{|c|c|c|c|c|c|c|c|c|c|}
\hline Datum / Uhrzeit & \begin{tabular}{|l|l}
$\mathrm{N}$ \\
\end{tabular} & Ber. & $30 \mathrm{~cm}$ & $40 \mathrm{~cm}$ & $50 \mathrm{~cm}$ & $60 \mathrm{~cm}$ & $70 \mathrm{~cm}$ & $80 \mathrm{~cm}$ & $90 \mathrm{~cm}$ \\
\hline $15.10 .9610: 00$ & 9,9 & 10 & 0 & & & & & & \\
\hline $16.10 .9610: 00$ & 15,4 & 10 & & & & & & & \\
\hline 17.10.96 10:00 & & 0 & 24,7 & 13,8 & 16,6 & 17,5 & 39,0 & 32,9 & 37, \\
\hline $.9615: 00$ & & 0 & 0,1 & 0,0 & 0,2 & 0,4 & 0,3 & 0,4 & 6,8 \\
\hline 23.10.96 08:00 & 22,2 & 0 & 26,3 & 10,0 & 8,8 & 10,3 & 39,0 & 42,8 & 40,8 \\
\hline $25.10 .9617: 30$ & 0 & 0 & 0,0 & 0,0 & 0,0 & 0,0 & 0,0 & 0,0 & 0,6 \\
\hline $.9615: 00$ & 4,9 & 0 & 0,0 & 0,1 & 0,0 & 0,1 & 0,0 & 0,2 & 0,2 \\
\hline $.9613: 00$ & & 0 & 0,3 & 0,0 & 0,0 & 0,6 & 0,0 & 0,0 & 0,2 \\
\hline $1.9610: 00$ & 11,4 & 0 & 1,5 & 0,8 & 0,7 & 1,7 & 1,1 & 0,4 & 2,2 \\
\hline .96 09:00 & 8,7 & 0 & 1,1 & 2,0 & 1,5 & 2,4 & 2,4 & 1,8 & 4,0 \\
\hline $.9608: 30$ & & 0 & 1,8 & 13,3 & 22,6 & 20,8 & 32,8 & 38,5 & 39 \\
\hline $608: 30$ & 30,9 & 0 & 0,3 & 0,2 & 0,0 & 1,0 & 0,9 & 1,0 & 4 \\
\hline $.9608: 30$ & 16,5 & 0 & 2,8 & 12,3 & 13,9 & 15,1 & 34,2 & 24,8 & 24,7 \\
\hline $2.9608: 30$ & 8,5 & 0 & 1,4 & 1,6 & 8,2 & 8,8 & 34,9 & 39,0 & 26,0 \\
\hline 30.1 & & 0 & 37,7 & 8,0 & 7,2 & 32,4 & 36,0 & 30,7 & 36, \\
\hline $9713: 30$ & & 0 & 4,2 & 0,0 & 0,4 & 2,2 & 5,1 & 1,7 & 2,4 \\
\hline $10.02 .9714: 00$ & 25,7 & 0 & 17,0 & 0,0 & 5,2 & 0,0 & 0,0 & 0,0 & 5,4 \\
\hline 14.02.97 13:00 & & 0 & 0,0 & 14,0 & 20,0 & 27,5 & 38,4 & 43,8 & 36,8 \\
\hline$: 00$ & 10,2 & 0 & 3 & 1,5 & 1,9 & 9,3 & 4,7 & 7 & 10,0 \\
\hline $.9713: 00$ & 8,6 & 0 & 2,0 & 4,0 & 5,2 & 3,7 & 4,4 & 11,0 & 16,1 \\
\hline $.9707: 40$ & 18,8 & 0 & 9,2 & 5,7 & 6,7 & 10,4 & 10,6 & 13,1 & 8,2 \\
\hline $.9707: 40$ & 12,7 & 0 & 7 & 7, & 7 & 11,1 & & 3 & 11,7 \\
\hline 7:40 & 0,4 & 0 & 0,6 & 1 , & 1,2 & 4 & 1,2 & 12,7 & 6,0 \\
\hline 97 16:00 & & 0 & 0,0 & 0,0 & 0,0 & 0,7 & 0,5 & 0,2 & 6,8 \\
\hline 97 07:00 & & 1 & 0,0 & 0,0 & 0,0 & 0,1 & 0 & 0 & 1, \\
\hline$: 00$ & 0,3 & 4 & 0, & 0,0 & 0,0 & 0,0 & 0,0 & 0,0 & 1,3 \\
\hline 97 10:00 & & 6 & 0,0 & 0,0 & 0,0 & 0,0 & 0,0 & 0,0 & 0,0 \\
\hline 97 08:00 & & 5 & 0,0 & 0,0 & 0,0 & 1, & 1,1 & 0,4 & 1,2 \\
\hline 97 07:40 & 0,1 & 5 & 1 , & 0, & 0 , & 4,0 & 0,6 & 3 , & 2,4 \\
\hline & & 5 & 1 & & & 4,7 & & 4,3 & 2,8 \\
\hline$: 30$ & & 5 & 0,7 & 0,8 & 0,6 & 3,4 & 2,5 & 2,4 & 2,6 \\
\hline $.9707: 30$ & 0,1 & 5 & 0, & 2,0 & 1, & 4,5 & 3,2 & 3 & 3,4 \\
\hline 97 08:40 & & 5 & 0,8 & 0 , & 0,7 & 3 , & & 2,7 & 2,9 \\
\hline$: 10$ & & 5 & 10 & 0,9 & 0,7 & 3,5 & 2,8 & 3,4 & 2,5 \\
\hline 7 07:45 & & 5 & 1,4 & 1,5 & 1,4 & 3,8 & & 4,0 & 3,5 \\
\hline 7 08:00 & & 5 & 1,1 & 0,4 & 1,1 & 4,1 & 3,6 & 4 & 3,6 \\
\hline 7 06:30 & & 0 & 0,4 & 0,9 & 0,5 & 3 & 2,7 & 3,3 & 2,3 \\
\hline 97 08:00 & 1,2 & 5 & & 2,4 & 2,2 & & 4,0 & 4,6 & 4,4 \\
\hline 97 07:40 & 3,2 & 5 & 1,0 & 3,4 & 4,0 & 6,9 & 6,0 & 7,0 & 5,8 \\
\hline $.9708: 15$ & 13 & 5 & 13,8 & 11,7 & 13,1 & 12,4 & 25,2 & 35,2 & 24,6 \\
\hline $.9712: 00$ & 0,1 & 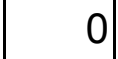 & 4,9 & 4,5 & 4,4 & 7,6 & & 33,8 & 11,8 \\
\hline $.9707: 50$ & 4,7 & 0 & 0,0 & 0,0 & 0,0 & 0,8 & 0,5 & 0,0 & 2,4 \\
\hline 20.03.97 08:10 & 1,7 & 0 & 0,9 & 0,8 & 0,8 & 4,4 & 2,9 & 1,1 & 0,5 \\
\hline $21.03 .9708: 10$ & 1,6 & 0 & 0,0 & 0,8 & 0,8 & 1,3 & 1,2 & 0,0 & 2,8 \\
\hline $22.03 .9711: 50$ & 2,2 & 0 & 0,0 & 0,0 & 0,0 & 1,3 & 1,2 & 0,6 & 2,2 \\
\hline
\end{tabular}




\begin{tabular}{|c|c|c|c|c|c|c|c|c|c|}
\hline Datum / Uhrzeit & $\mathrm{N}$ & Ber. & $30 \mathrm{~cm}$ & $40 \mathrm{~cm}$ & $50 \mathrm{~cm}$ & $60 \mathrm{~cm}$ & $70 \mathrm{~cm}$ & $80 \mathrm{~cm}$ & $90 \mathrm{~cm}$ \\
\hline $23.03 .9711: 10$ & 1,1 & 0 & 0,0 & 0,0 & 0,0 & 0,4 & 0,6 & 0,2 & 1,2 \\
\hline $24.03 .9707: 40$ & & 0 & 0,0 & 0,0 & 0,0 & 0,0 & 0,2 & 0,0 & 0,5 \\
\hline $25.03 .9707: 45$ & 0,8 & 0 & 0,0 & 0,0 & 0,0 & 0,1 & 0,0 & 0,0 & 0,0 \\
\hline $27.03 .9708: 30$ & 1,3 & 0 & 0,0 & 0,0 & 0,0 & 0,0 & 0,8 & 0,0 & 1,0 \\
\hline 02.04.97 06:40 & 9,2 & 0 & 0,0 & 0,8 & 0,8 & 2,5 & 1,2 & 0,9 & 0,3 \\
\hline 08.04.97 12:30 & 12,1 & 10 & 1,7 & 1,0 & 0,5 & 4,3 & 2,7 & 3,2 & 2,2 \\
\hline 10.04.97 10:10 & & 10 & 3,0 & 1,9 & 0,9 & 3,8 & 2,9 & 4,9 & 2,3 \\
\hline 11.04.97 08:00 & & 0 & 4,5 & 3,2 & 2,2 & 5,5 & 4,4 & 5,2 & 2,8 \\
\hline $14.04 .9712: 40$ & 2,7 & 5 & 0,0 & 0,0 & 0,0 & 0,8 & 0,7 & 0,7 & 1,0 \\
\hline $15.04 .9711: 00$ & 0,5 & 5 & 0,4 & 0,0 & 0,0 & 0,3 & 0,0 & 0,0 & 0,0 \\
\hline $16.04 .9712: 40$ & & 5 & 3,3 & 2,0 & 0,9 & 4,1 & 2,9 & 3,7 & 1,1 \\
\hline 17.04.97 11:00 & & 5 & 3,0 & 2,8 & 2,5 & 4,9 & 3,6 & 4,4 & 2,1 \\
\hline 18.04.97 10:00 & & 5 & 3,5 & 2,9 & 2,0 & 4,6 & 3,8 & 4,4 & 2,9 \\
\hline 19.04.97 11:50 & 0,6 & 5 & 3,0 & 3,2 & 2,4 & 5,1 & 4,8 & 5,1 & 3,6 \\
\hline $20.04 .9707: 50$ & & 5 & 3,2 & 1,7 & 2,0 & 4,5 & 3,9 & 3,8 & 3,2 \\
\hline 21.04.97 08:50 & 0 & 5 & 2,4 & 2,6 & 2,3 & 5,6 & 4,6 & 5,1 & 4,8 \\
\hline $22.04 .9711: 15$ & 4,1 & 5 & 3,1 & 2,5 & 1,9 & 4,8 & 4,7 & 4,7 & 3,6 \\
\hline $23.04 .9707: 50$ & 0 & 5 & 2,5 & 2,4 & 1,3 & 4,4 & 3,3 & 4,3 & 3,3 \\
\hline $24.04 .9711: 00$ & 0 & 2 & 3,0 & 2,6 & 1,8 & 4,9 & 4,3 & 2,3 & 4,9 \\
\hline $25.04 .9708: 55$ & 0 & 0 & 0,0 & 0,0 & 0,0 & 1,0 & 1,1 & 1,0 & 1,1 \\
\hline $28.04 .9710: 50$ & 5,5 & 2 & 0,1 & 0,1 & 0,1 & 0,1 & 0,3 & 0,3 & 0,6 \\
\hline $29.04 .9711: 30$ & 4,3 & 2 & 0,0 & 0,0 & 0,0 & 0,0 & 0,0 & 0,0 & 0,0 \\
\hline 05.05.97 09:10 & 2,7 & 2 & 0,0 & 0,0 & 0,0 & 0,9 & 0,0 & 0,0 & 0,0 \\
\hline 13.05.97 10:00 & 41 & 0 & 0,0 & 0,0 & 0,0 & 0,0 & 32,2 & 1,3 & 0,7 \\
\hline $26.05 .9713: 00$ & 44,7 & 0 & 0,0 & 0,0 & 0,0 & 0,0 & 1,2 & 0,1 & 0,0 \\
\hline $02.06 .9715: 30$ & 0 & 0 & 0,0 & 0,0 & 1,8 & 0,0 & 0,0 & 0,0 & 0,0 \\
\hline 10.06.97 13:00 & 1 & 10 & 0,0 & 0,0 & 0,0 & 0,0 & 0,4 & 0,0 & 0,0 \\
\hline 12.06.97 19:00 & 0 & 10 & 0,9 & 0,2 & 1,0 & 2,2 & 3,2 & 1,8 & 0,7 \\
\hline 13.06.97 10:00 & 0 & 10 & 2,9 & 3,2 & 4,5 & 5,5 & 3,7 & 5,9 & 4,0 \\
\hline 16.06.97 10:00 & 3 & 10 & 3,6 & 4,0 & 3,5 & 6,8 & 7,1 & 8,2 & 7,6 \\
\hline 18.06.97 10:00 & 0 & 10 & 2,9 & 2,7 & 1,9 & 5,2 & 4,1 & 4,9 & 5,4 \\
\hline 19.06.97 10:00 & 1,8 & 10 & 3,4 & 4,9 & 3,2 & 5,8 & 5,5 & 4,7 & 4,8 \\
\hline 20.06.97 10:00 & 0 & 10 & 5,5 & 6,1 & 4,1 & 7,2 & 11,1 & 14,2 & 9,7 \\
\hline 21.06.97 10:00 & 1,8 & 0 & 5,1 & 4,4 & 3,8 & 6,7 & 6,6 & 13,2 & 9,0 \\
\hline $25.06 .9710: 00$ & 26,4 & 0 & 6,1 & 5,3 & 5,5 & 9,0 & 9,6 & 27,4 & 14,6 \\
\hline
\end{tabular}


Anhang B: Chlorid-Konzentrationen $\left[\mathrm{mg}^{\cdot \mathrm{L}^{-1}}\right]$ und Perkolationsrate $\left[\mathrm{mm}^{\cdot \mathrm{d}^{-1}}\right]$ aus drei Laborlysimetern der Länge 30 (Ly 1), 53,1 (Ly 2) und 67,5 (Ly 3) (Oberfläche $A=1075 \mathrm{~cm}^{2}$ )

\begin{tabular}{|c|c|c|c|c|c|c|}
\hline \multirow[b]{2}{*}{ Datum / Uhrzeit } & \multicolumn{3}{|c|}{ Perkolationsrate $\left[\mathrm{mm}^{-1}{ }^{-1}\right]$} & \multicolumn{3}{|c|}{$\begin{array}{l}\text { Chlorid-Konzentrationen } \\
{\left[\mathrm{mg} \mathrm{L}^{-1}\right]}\end{array}$} \\
\hline & Ly 1 & Ly 2 & Ly 3 & Ly 1 & Ly 2 & Ly 3 \\
\hline $22.04 .9808: 00$ & 0,0 & 0,0 & 0,0 & 5 & 5 & 5 \\
\hline $22.04 .9811: 00$ & 15,0 & 14,1 & 15,4 & 6,76 & 5,54 & 4,56 \\
\hline $22.04 .9814: 00$ & 14,9 & 14,0 & 15,2 & 93,71 & 6,245 & 5,765 \\
\hline $22.04 .9817: 00$ & 15,1 & 14,2 & 15,4 & 245,60 & 6,59 & 5,495 \\
\hline $22.04 .9820: 00$ & 15,0 & 14,6 & 15,4 & 352,39 & 7,23 & 5,615 \\
\hline 22.04.98 23:00 & 14,8 & 14,2 & 15,1 & 418,04 & 8,79 & 5,685 \\
\hline $23.04 .9802: 00$ & 15,0 & 14,3 & 15,3 & 450,77 & 16,06 & 6,595 \\
\hline 23.04.98 05:00 & 15,0 & 14,4 & 15,2 & 478,86 & 30,355 & 6,785 \\
\hline $23.04 .9808: 00$ & 14,9 & 14,3 & 15,2 & 520,41 & 49,93 & 8,23 \\
\hline $23.04 .9811: 00$ & 14,9 & 14,2 & 15,2 & 570,27 & 70,565 & 10,295 \\
\hline $23.04 .9814: 00$ & 15,0 & 14,2 & 15,2 & 598,97 & 94,5 & 13,75 \\
\hline $23.04 .9817: 00$ & 14,6 & 14,7 & 14,7 & 619,89 & 120,57 & 12,655 \\
\hline 3 20:00 & 15,1 & 16,2 & 15,0 & 637,88 & 151,185 & 23,325 \\
\hline 23.04.98 23:00 & 15,0 & 16,1 & 15,0 & 650,22 & 185,02 & 30,545 \\
\hline $24.04 .9802: 00$ & 15,0 & 15,7 & 15,0 & 654,81 & 216,765 & 35,785 \\
\hline 24.0 & 14,9 & 15,7 & 15,0 & 672,34 & 242,12 & 41,185 \\
\hline $24.04 .9808: 00$ & 14,9 & 15,6 & 15,0 & 662,64 & 263,405 & 47,84 \\
\hline $24.04 .9811: 00$ & 14,9 & 15,6 & 14,9 & 663,98 & 285,615 & 55,335 \\
\hline $24.04 .9814: 00$ & 15,0 & 15,6 & 15,0 & 692,84 & 306,81 & 64,17 \\
\hline 24.04 .9 & 14,7 & 15,4 & 14,7 & 708,38 & 321,56 & 71,77 \\
\hline $24.04 .9820: 00$ & 14,8 & 15,2 & 14,8 & 718,87 & 335,79 & 79,15 \\
\hline $24.04 .9823: 00$ & 14,7 & 15,2 & 14,8 & 728,36 & 351,86 & 88,16 \\
\hline $2: 00$ & 14,7 & 15,1 & 14,8 & 749,2 & 366,44 & 98,47 \\
\hline 25.04 .9 & 14,7 & 15,0 & 14,8 & 760,07 & 383,81 & 113,84 \\
\hline $25.04 .9814: 00$ & 14,8 & 14,8 & 14,9 & 776,39 & 412,81 & 6,14 \\
\hline $25.04 .9820: 00$ & 15,5 & 17,9 & 15,3 & 790,43 & 442,77 & 162,58 \\
\hline 26.04 .9 & 14,5 & 14,4 & 15,0 & 802,65 & 477,12 & 186,57 \\
\hline 26.04.98 08:00 & 15,7 & 14,2 & 14,9 & 805,48 & 492,95 & 209,04 \\
\hline $26.04 .9814: 00$ & 15,8 & 14,6 & 14,9 & 821,3 & 510,41 & 230,34 \\
\hline 26.04 .98 17:00 & 15,9 & 15,5 & 14,8 & 832,5 & 515,59 & 247,81 \\
\hline 26.04.98 20:00 & 15,7 & 14,9 & 14,8 & 834,67 & 530,3 & 260,3 \\
\hline $27.04 .9802: 00$ & 15,7 & 15,0 & 14,8 & 845,84 & 550,76 & 277,81 \\
\hline $27.04 .9808: 00$ & 15,3 & 14,9 & 15,2 & 853,15 & 569,65 & 298,95 \\
\hline $27.04 .9814: 00$ & 15,1 & 26,0 & 14,9 & 865,72 & 594,17 & 321,1 \\
\hline $27.04 .9820: 00$ & 15,1 & 11,1 & 15,5 & 865,77 & 621,51 & 354,52 \\
\hline $28.04 .9802: 00$ & 15,0 & 14,5 & 15,6 & 874,73 & 631,69 & 375,13 \\
\hline $28.04 .9808: 00$ & 14,7 & 14,3 & 15,3 & 872,58 & 632,7 & 389,71 \\
\hline $28.04 .9814: 00$ & 14,8 & 14,2 & 14,5 & 870,46 & 640,33 & 400,42 \\
\hline $28.04 .9820: 00$ & 14,6 & 14,0 & 14,2 & 870,44 & 653,3 & 418,78 \\
\hline $29.04 .9802: 00$ & 14,0 & 13,8 & 14,2 & 869,43 & 660,44 & 430,82 \\
\hline $29.04 .9808: 00$ & 14,3 & 14,1 & 14,6 & 877,34 & 665,45 & 439,91 \\
\hline $29.04 .9814: 00$ & 14,6 & 13,4 & 15,1 & 876,99 & 672,18 & 460,16 \\
\hline $29.04 .9820: 00$ & 14,5 & 14,6 & 15,2 & 875,63 & 687,24 & 482,4 \\
\hline
\end{tabular}




\begin{tabular}{|c|c|c|c|c|c|c|}
\hline Datum / Uhrzeit & Perkolat & rate [ 1 & $\left.d^{-1}\right]$ & Chlori & $\begin{array}{l}\text { Konzentr } \\
\mathrm{mg}^{-1} \mathrm{~L}^{-1}\end{array}$ & onen \\
\hline $30.04 .9802: 00$ & 14,4 & 14,6 & 15,1 & 883,95 & 690,51 & 496,6 \\
\hline $30.04 .9808: 00$ & 14,7 & 14,9 & 14,8 & 879,64 & 700,2 & 508,6 \\
\hline $30.04 .9814: 00$ & 14,7 & 15,0 & 15,8 & 878,53 & 703,51 & 523 \\
\hline 30.04 .98 20:00 & 14,6 & 15,0 & 15,9 & 877,18 & 716,53 & 552,14 \\
\hline 01.05.98 09:00 & 15,0 & 15,0 & 14,9 & 898,34 & 724,11 & 566,65 \\
\hline 01.05.98 20:00 & 15,7 & 15,5 & 16,2 & 908,24 & 735,08 & 584,8 \\
\hline 02.05.98 08:00 & 15,5 & 15,4 & 15,6 & 906,54 & 761,34 & 612,2 \\
\hline 02.05.98 20:00 & 14,5 & 14,8 & 15,1 & 907,84 & 759,14 & 617,89 \\
\hline 03.05.98 08:00 & 14,6 & 15,4 & 15,5 & 908,17 & 771,01 & 639,44 \\
\hline 03.05.98 19:30 & 15,3 & 15,5 & 14,9 & 914,04 & 787,25 & 636,6 \\
\hline $04.05 .9807: 30$ & 15,2 & 15,3 & 14,8 & 911,31 & 786,37 & 651,84 \\
\hline 04.05 .98 19:45 & 14,6 & 15,0 & 14,7 & 916,12 & 814,12 & 677,6 \\
\hline $05.05 .9807: 30$ & 14,1 & 14,7 & 14,3 & 931,54 & 815,75 & 699,74 \\
\hline 05.05.98 19:30 & 13,1 & 14,4 & 14,5 & 936,49 & 820,7 & 704,67 \\
\hline $06.05 .9807: 30$ & 14,1 & 14,6 & 14,8 & 938,77 & 828,59 & 715,31 \\
\hline $06.05 .9819: 45$ & 13,8 & 14,9 & 15,3 & 942,25 & 833,3 & 726,98 \\
\hline $5.9808: 00$ & 14,5 & 14,6 & 14,8 & 947,94 & 838,33 & 747,89 \\
\hline 07.05.98 19:30 & 16,9 & 16,4 & 16,3 & 951,16 & 849,72 & 763,13 \\
\hline $08.05 .9807: 30$ & 15,8 & 15,3 & 15,1 & 950,31 & 852,06 & 766,89 \\
\hline $9: 45$ & 15,6 & 15,1 & 14,9 & 955,67 & 5,17 & 776,27 \\
\hline $09.05 .9807: 45$ & 15,4 & 14,9 & 14,6 & 950,68 & 857,4 & 790,1 \\
\hline 09.05.98 19:45 & 15,4 & 14,9 & 14,6 & 952,31 & 860,06 & 792,68 \\
\hline $10.05 .9807: 45$ & 15,3 & 14,8 & 14,5 & 953,91 & 855,67 & 816,49 \\
\hline $10.05 .9819: 45$ & 15,2 & 14,7 & 14,4 & 955,61 & 863,83 & 815,73 \\
\hline $11.05 .9807: 45$ & 15,2 & 14,7 & 14,4 & 958,78 & 865,93 & 817,77 \\
\hline $11.05 .9819: 45$ & 15,1 & 14,6 & 14,3 & 955,47 & 871,76 & 818,35 \\
\hline $12.05 .9807: 45$ & 15,0 & 14,6 & 14,3 & 957,25 & 868,16 & 859,92 \\
\hline $12.05 .9819: 45$ & 15,0 & 14,5 & 14,2 & 950,23 & 875,32 & 844,71 \\
\hline $13.05 .9807: 45$ & 15,2 & 14,7 & 14,4 & 957,65 & 864,69 & 847,84 \\
\hline $14.05 .9807: 45$ & 14,8 & 14,3 & 14,0 & 964,4 & 889,4 & 760,52 \\
\hline $15.05 .9807: 45$ & 15,3 & 14,6 & 14,7 & 971 & 878,09 & 750 \\
\hline $16.05 .9807: 45$ & 14,6 & 13,9 & 14,3 & 961,12 & 886,5 & 824,08 \\
\hline $17.05 .9807: 45$ & 13,9 & 13,1 & 13,6 & 967,9 & 872,36 & 862,36 \\
\hline $18.05 .9807: 45$ & 13,5 & 12,8 & 13,3 & 955,16 & 847,79 & 867,68 \\
\hline 19.05.98 07:45 & 12,8 & 12,0 & 12,6 & 964,72 & 896,22 & 870,66 \\
\hline $20.05 .9807: 45$ & 15,8 & 14,6 & 15,7 & 969,1 & 871,72 & 888,4 \\
\hline $21.05 .9807: 45$ & 16,3 & 6,9 & 16,2 & 970,18 & 821,69 & 894,78 \\
\hline 22.05 .98 07:45 & 16,2 & 17,7 & 16,1 & 975,68 & 900,27 & 863,41 \\
\hline $23.05 .9807: 45$ & 16,0 & 15,0 & 16,2 & 977,89 & 895,66 & 886,85 \\
\hline $24.05 .9807: 45$ & 15,9 & 14,7 & 16,1 & 971,75 & 924,01 & 901,88 \\
\hline $25.05 .9807: 45$ & 15,9 & 14,6 & 16,0 & 984,23 & 938,35 & 912,67 \\
\hline $26.05 .9807: 45$ & 15,8 & 34,9 & 16,0 & 995,75 & 968,62 & 920,89 \\
\hline $27.05 .9807: 45$ & 15,6 & 15,4 & 15,8 & 999,92 & 949,62 & 942,01 \\
\hline $28.05 .9807: 45$ & 14,7 & 14,4 & 15,2 & 984,97 & 958,43 & 926,67 \\
\hline $29.05 .9807: 45$ & 14,4 & 13,7 & 14,7 & 991,73 & 970,53 & 934,65 \\
\hline $30.05 .9807: 45$ & 14,8 & 14,0 & 14,8 & 977,69 & 970,02 & 938,56 \\
\hline $31.05 .9807: 45$ & 14,8 & 14,0 & 14,8 & 980,93 & 964,75 & 939,16 \\
\hline
\end{tabular}




\begin{tabular}{|c|c|c|c|c|c|c|}
\hline & \multicolumn{3}{|c|}{ Perkolationsrate $\left[\mathrm{mm}^{-1}{ }^{-1}\right]$} & \multicolumn{3}{|c|}{$\begin{array}{l}\text { Chlorid-Konzentrationen } \\
{\left[\mathrm{mg}^{-1} \mathrm{~L}^{-1}\right]}\end{array}$} \\
\hline $01.06 .9807: 45$ & 15,4 & 14,4 & 15,3 & 978,97 & 965,05 & 940,51 \\
\hline 02.06.98 07:45 & 15,4 & 14,2 & 15,3 & 977,7 & 958,72 & 945,71 \\
\hline $03.06 .9807: 45$ & 15,7 & 14,5 & 15,6 & 989,58 & 963,74 & 951,71 \\
\hline $04.06 .9807: 45$ & 15,0 & 13,8 & 14,9 & 983,2 & 961,47 & 962,29 \\
\hline 05.06.98 07:45 & 15,5 & 13,9 & 15,5 & 978,9 & 964,47 & 956,72 \\
\hline 06.06.98 07:45 & 15,5 & 14,7 & 15,4 & 981,84 & 977,36 & 955,21 \\
\hline $07.06 .9807: 45$ & 15,7 & 14,5 & 15,4 & 976,2 & 953,85 & 954,23 \\
\hline 08.06.98 07:45 & 15,7 & 14,3 & 15,5 & 982,09 & 954,55 & 967,27 \\
\hline 09.06.98 07:45 & 15,8 & 14,8 & 15,5 & 979,92 & 959,17 & 958,61 \\
\hline 16.06.98 08:00 & 15,3 & 15,1 & 15,1 & 985,34 & 971,95 & 968 \\
\hline 23.06.98 08:00 & 15,0 & 15,8 & 14,9 & 992,37 & 969,96 & 983,44 \\
\hline 30.06 .9 & 14,9 & 14,8 & 14,9 & 985,35 & 977,05 & 972,62 \\
\hline 07.07.98 08:00 & 14,5 & 14,8 & 15,2 & 981,47 & 969,89 & 980,88 \\
\hline $14.07 .9808: 00$ & 13,9 & 14,8 & 13,7 & 997,82 & 988,27 & 990,7 \\
\hline 21.07.98 08:00 & 16,2 & 16,0 & 15,3 & 1010,1 & 997,86 & 1002,59 \\
\hline $28.07 .9808: 00$ & 15,4 & 14,6 & 15,2 & 1004,37 & 994,27 & 998,72 \\
\hline 04.08.98 08:00 & 15,0 & 14,9 & 14,8 & 1000,88 & 1000,86 & 1002,61 \\
\hline $11.08 .9808: 00$ & 14,9 & 15,0 & 14,8 & 1020,53 & 1002,98 & 1016,04 \\
\hline 18.08.98 08:00 & 14,8 & 14,2 & 14,9 & 1010,8 & 1001,9 & 1004,82 \\
\hline $25.08 .9808: 00$ & 14,3 & 15,9 & 14,9 & 999,085 & 997,005 & 996,9 \\
\hline 01.09.98 08:00 & 15,0 & 15,9 & 15,8 & 1003,49 & 1002,25 & 1010,66 \\
\hline 08.09.98 08:00 & 15,0 & 16,1 & 15,8 & 1006,54 & 1004,05 & 1003,34 \\
\hline $15.09 .9808: 00$ & 15,0 & 16,2 & 15,8 & 1012,54 & 1009,38 & 1007,45 \\
\hline $22.09 .9808: 00$ & 15,0 & 15,5 & 15,8 & 1014,9 & 1009,62 & 1009,64 \\
\hline
\end{tabular}


Anhang C: Nach MUNSELL-TAFELN bestimmte Bodenfarben vom CvVerwitterungsprofil und vom Pelosol-Akkumulationsprofil; I = Hauptfarbe; II = Nebenfarbe

\begin{tabular}{|c|c|c|c|c|}
\hline \multicolumn{5}{|c|}{ Pelosol Akkumulationsprofil } \\
\hline & \multicolumn{2}{|c|}{ Trockene Bestimmung } & \multicolumn{2}{|c|}{ Nasse Bestimmung } \\
\hline & 1 & II & I & II \\
\hline Tiefe [cm] & Munsell-Tab. & Munsell-Tab. & Munsell-Tab. & \\
\hline $0-10$ & 7,5YR 5/4 & & $5 Y R$ 3/4 & \\
\hline $10-20$ & $5 Y R$ 5/3 & & $5 Y R$ 4/3 & Gley 5GY 6/1 \\
\hline $20-30$ & $5 Y R$ 4/4 & Gley 5GY 7/1 & 5YR 3/4 & \\
\hline $30-40$ & $5 Y R$ 4/3 & & 5YR 3/3-4 & \\
\hline $40-50$ & $5 Y R \quad 4 / 3$ & & 5YR 3/3 & \\
\hline $50-60$ & $5 Y R \quad 4 / 3$ & & $5 Y R$ 3/4 & \\
\hline $60-70$ & 5YR 4/4 & & 5YR 3/4 & \\
\hline $70-80$ & $5 Y R \quad 4 / 3$ & Gley 5GY 7/1 & 5YR 3/4 & Gley 5GY 6/1 \\
\hline $80-90$ & 5YR 4/4 & Gley 5GY 7/1 & 5YR 3/3-4 & Gley 5GY 6/1 \\
\hline $90-100$ & 5YR 5/4 & Gley 5GY 7/1 & $5 Y R$ 4/3 & Gley 5GY 6/1 \\
\hline $100-110$ & 5YR 5/4 & Gley 5 GY 7/1 & $5 Y R$ 3/4 & Gley 5GY 6/1 \\
\hline $110-120$ & 5YR 5/4 & Gley 5GY 7/1 & $5 Y R$ 4/3 & Gley 5GY 6/1 \\
\hline $120-130$ & 5YR 5/4 & Gley 5GY 7/1 & $5 Y R \quad 4 / 3$ & Gley 5GY 6/1 \\
\hline $130-140$ & Gley 5GY 7/1 & $5 Y R$ 5/3 & Gley 5GY 6/1 & $5 Y R$ 4/3 \\
\hline $140-150$ & $5 Y R$ 5/3 & Gley 5GY 7/1 & $5 Y R$ 4/3 & Gley 5GY 6/1 \\
\hline $150-160$ & 5YR 4/4 & Gley N7 & 5YR 5/4 & Gley N6 \\
\hline $160-170$ & 5YR 4/4 & & $5 Y R$ 4/3 & \\
\hline $170-180$ & $5 Y R$ 4/3 & & $5 Y R$ 3/3 & \\
\hline $180-190$ & $5 Y R$ 4/3 & & $5 Y R 3 / 3$ & \\
\hline $190-200$ & $5 Y R \quad 4 / 3$ & & $5 Y R$ 3/2 & \\
\hline $200-210$ & Gley 5GY 7/1 & 5YR 4/3 & Gley 5GY 6/1 & 5YR 3/3 \\
\hline \multicolumn{5}{|c|}{ Verwitterungsprofil } \\
\hline & \multicolumn{2}{|c|}{ Trockene Bestimmung } & \multicolumn{2}{|c|}{ Nasse Bestimmung } \\
\hline & 1 & II & 1 & II \\
\hline Tiefe $[\mathrm{cm}]$ & Munsell-Tab. & Munsell-Tab. & Munsell-Tab. & \\
\hline $0-10$ & $5 Y R$ 4/4 & & 5YR 3/4 & \\
\hline $10-20$ & 5YR 4/4 & & 5YR 3/4 & \\
\hline $20-30$ & 5YR 4/4 & & 5YR 3/4 & Gley 5GY 6/1 \\
\hline $30-40$ & 5YR 5/4 & Gley 5GY 7/1 & 5YR 3/4 & Gley 5Y 6/1 \\
\hline $40-50$ & 5YR 4/4 & Gley 5GY 7/1 & 5YR 3/4 & Gley 5GY 5/1 \\
\hline $50-60$ & 5YR 4/6 & Gley 5GY 7/1 & 5YR 3/4 & \\
\hline $60-70$ & 5YR 3-4/4 & & 5YR 3/4 & \\
\hline $70-80$ & 5YR 4/4 & & 5YR 3/4 & Gley 5GY 6/1 \\
\hline $80-90$ & 5YR 4/4 & Gley 5GY 7/1 & 5YR 3/4 & Gley 5GY 6/1 \\
\hline $90-100$ & $5 Y R$ 4/3 & Gley 5GY 7/1 & 5YR 3/4 & \\
\hline $100-110$ & 5YR 4/6 & & 5YR 3/4 & Gley 5GY 6/1 \\
\hline $110-120$ & 5YR 4/4 & Gley 5GY 7/1 & 5YR 3/4 & 5YR 3/4 \\
\hline $120-130$ & Gley 5GY 7/1 & 5YR 4/4 & Gley 5GY 6/1 & Gley 5GY 6/1 \\
\hline $130-140$ & $5 Y R$ 4/3 & Gley 5GY 7/1 & $5 Y R 3 / 4$ & Gley 5GY 6/1 \\
\hline $140-150$ & 5YR 4/4 & Gley 5GY 7/1 & 5YR 3/4 & Gley 5GY 6/1 \\
\hline $150-160$ & 5YR 4/4 & Gley 5GY 7/1 & 5YR 3/4 & \\
\hline $160-170$ & 5YR 4/4 & & 5YR 3/4 & \\
\hline $170-180$ & 5YR 4/4 & Gley 5Y 7//1 & 5YR 3/4 & Gley 5GY 6/1 \\
\hline $180-190$ & 5YR 4/4 & Gley 5GY 7/1 & 5YR 3/4 & Gley 5GY 6/1 \\
\hline $190-200$ & 5YR 4/4 & Gley $5 Y$ 7//1 & 5YR 3/4 & Gley 5GY 6/1 \\
\hline $200-210$ & 10R 4/2 & & 10R 4/2 & \\
\hline
\end{tabular}


Anhang C: Wassergehalts-Saugspannungsbeziehung von einem PelosolAkkumulationsprofil und einem Cv-Verwitterungsprofil aus Röt; Tiefenangabe ist jeweils die Mitte des Stechzylinders in $\mathrm{cm} ; \mathrm{P}=$ Pelosol-Akkumulationsprofil; $\mathrm{Cv}=$ Verwitterungsprofil; Angaben in $\%$-Vol.

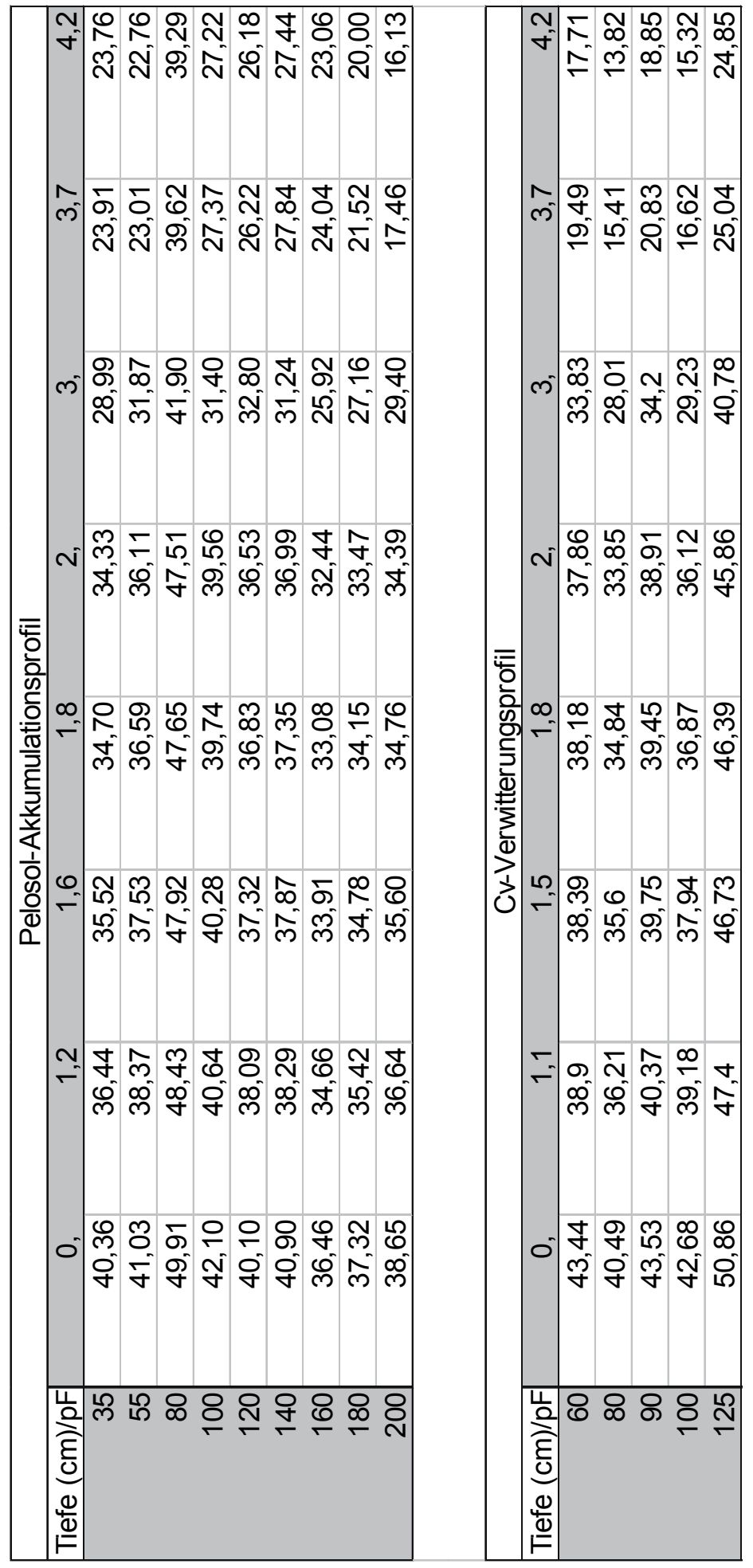


Anhang D: Korngrößenanalysen der bei pF-Messungen verwendeten Stechzylinder (Anhang D) von einem Pelosol-Akkumulationsprofil und einem Cv-Verwitterungsprofil aus Röt; Angaben in Gew.-\%;

\begin{tabular}{|r|r|r|r|r|r|r|r|}
\hline \multicolumn{7}{|c|}{ Ohne Zerstörung der Kittsubstanzen } \\
\hline Boden- & \multicolumn{3}{|c|}{ Sand } & \multicolumn{3}{|c|}{ Schluff } & \multicolumn{1}{c|}{ Ton } \\
\cline { 2 - 8 } tiefe & $2000-$ & $630-$ & $200-63$ & $63-36$ & $36-20$ & $20-2$ & $<2 \mu \mathrm{m}$ \\
$(\mathrm{cm})$ & $630 \mu \mathrm{m}$ & $200 \mu \mathrm{m}$ & $\mu \mathrm{m}$ & $\mu \mathrm{m}$ & $\mu \mathrm{m}$ & $\mu \mathrm{m}$ & \\
\hline 35 & 1,93 & 0,73 & 2,55 & 4,79 & 5,21 & 47,45 & 37,34 \\
55 & 2,40 & 0,65 & 2,00 & 4,30 & 4,90 & 48,60 & 37,14 \\
80 & 2,26 & 0,91 & 6,66 & 8,70 & 10,04 & 32,76 & 38,67 \\
100 & 0,74 & 0,20 & 1,34 & 2,73 & 3,42 & 33,50 & 58,08 \\
120 & 0,58 & 1,01 & 10,81 & 10,81 & 11,07 & 35,33 & 30,40 \\
140 & 1,34 & 1,72 & 12,58 & 11,88 & 11,77 & 28,55 & 32,15 \\
160 & 1,59 & 0,66 & 23,55 & 18,11 & 10,15 & 24,09 & 21,84 \\
180 & 0,15 & 0,21 & 21,65 & 20,77 & 13,06 & 25,24 & 18,92 \\
200 & 0,50 & 0,43 & 2,61 & 11,11 & 16,45 & 49,41 & 19,49 \\
60 & 0,25 & 0,30 & 4,96 & 11,67 & 12,42 & 42,26 & 28,14 \\
80 & 0,05 & 0,10 & 13,03 & 19,48 & 14,09 & 32,71 & 20,53 \\
90 & 5,40 & 3,03 & 6,00 & 5,64 & 7,46 & 45,54 & 26,93 \\
100 & 0,60 & 0,93 & 6,15 & 0,71 & 8,12 & 35,08 & 48,42 \\
125 & 1,07 & 0,19 & 4,58 & 11,91 & 19,81 & 40,69 & 21,76 \\
\hline
\end{tabular}

\begin{tabular}{|c|c|c|c|c|c|c|c|}
\hline \multicolumn{8}{|c|}{ mit Zerstörung der Kittsubstanzen } \\
\hline \multirow{2}{*}{$\begin{array}{c}\text { Boden- } \\
\text { tiefe } \\
(\mathrm{cm})\end{array}$} & \multicolumn{3}{|c|}{ Sand } & \multicolumn{3}{|c|}{$\begin{array}{r}\text { Schluff } \\
\end{array}$} & \multirow{2}{*}{\begin{tabular}{|c|} 
Ton \\
$<2 \mu \mathrm{m}$
\end{tabular}} \\
\hline & $\begin{array}{l}2000- \\
630 \mu \mathrm{m}\end{array}$ & $\begin{array}{l}630- \\
200 \mu \mathrm{m}\end{array}$ & $\begin{array}{l}200-63 \\
\mu m\end{array}$ & $\begin{array}{l}63-36 \\
\mu m\end{array}$ & $\begin{array}{l}36-20 \\
\mu m\end{array}$ & $\begin{array}{l}20-2 \\
\mu m\end{array}$ & \\
\hline 35 & 0,06 & 1,06 & 1,89 & 5,14 & 4,79 & 43,91 & 43,14 \\
\hline 55 & 0,07 & 0,20 & 1,33 & 5,72 & 7,85 & 45,38 & 39,45 \\
\hline 80 & 1,50 & 0,78 & 5,72 & 8,32 & 10,92 & 39,27 & 33,49 \\
\hline 100 & 0,25 & 0,20 & 1,36 & 2,37 & 3,27 & 44,99 & 47,56 \\
\hline 120 & 0,66 & 0,51 & 8,85 & 9,31 & 11,25 & 39,59 & 29,82 \\
\hline 140 & 1,11 & 1,47 & 12,48 & 12,53 & 9,95 & 35,37 & 27,08 \\
\hline 160 & 0,35 & 0,52 & 24,15 & 17,49 & 9,15 & 27,45 & 20,90 \\
\hline 180 & 0,15 & 0,65 & 18,00 & 20,10 & 15,00 & 26,55 & 19,55 \\
\hline 200 & 0,63 & 0,23 & 24,15 & 9,97 & 17,87 & 39,08 & 8,07 \\
\hline 60 & 0,25 & 0,20 & 5,35 & 12,15 & 12,10 & 44,25 & 25,70 \\
\hline 80 & 0,10 & 0,05 & 16,05 & 24,45 & 11,75 & 30,70 & 16,90 \\
\hline 90 & 2,41 & 4,52 & 8,33 & 7,17 & 8,18 & 38,79 & 30,61 \\
\hline 100 & 0,57 & 0,57 & 5,52 & 6,61 & 7,92 & 37,40 & 41,41 \\
\hline 125 & 0,16 & 0,36 & 4,37 & 16,12 & 14,77 & 36,92 & 27,30 \\
\hline
\end{tabular}




\section{Dank}

Mein Dank gilt allen, die zum Zustandekommen dieser Arbeit beigetragen haben:

Herrn Prof. Dr. B. Meyer für die Überlassung des Themas und das ständige Interesse am Fortgang der Arbeit.

Herrn Prof. Dr. G. Wessolek danke ich für die freundliche Übernahme des Korreferates.

Die praktische Betreuung hatte Herr Dr. K.-W. Becker, dem ich ganz besonders für die ständige Gesprächsbereitschaft und Hilfe bei der Durchführung im Felde danken möchte.

Herrn Dr. R. Well und Herrn Dr. C Ahl sei für die kritische Durchsicht des Manuskriptes gedankt.

Mit dem Auf- und Abbau der Lysimeteranlage im Feld hatten Herr G. Benseler, Herr H. Linne und Herr G. Geveke zu tun, denen ich dafür danken möchte.

Herrn W. Winter danke ich für den Bau der Plattenlysimeter.

Für die Hilfe bei den Feldarbeiten danke ich Frau S. Enzmann, Frau U. Kierbaum und vor allem Frau U. Hill.

Die Durchführung der Korngrößenanalyse wurde mit größter Sorgfalt von Frau I. Ostermeyer durchgeführt.

Frau K. Schmidt danke ich für die zahlreichen Chloridanalysen und Frau S.

Richter für die Hilfe bei den $\mathrm{pF}-$ Messungen.

Herrn P. Gernandt danke ich für die Hilfe beim Lösen der graphischen Probleme.

Dank gilt meiner Frau Katrin für die vielen Aufmunterungen im Verlauf der Arbeit.

Besonders dankbar bin ich meinen Eltern, die mir das Studium und letztlich auch die Dissertation ermöglichten.

Zum Schluss sei meiner Frau und meinen Eltern für den Versuch gedankt, mir die neuen Rechtschreibregeln näher zu bringen. 